\title{
Parameter uncertainty methods in evaluating a lumped hydrological model
}

\author{
Método de incertidumbre paramétrica en la evaluación de un modelo hidrológico agregado
}

Fecha de entrega: 8 de octubre 2012

Fecha de aceptación: 12 de diciembre 2012

\section{Jairo Diaz-Ramirez, Rene Camacho, William McAnally and James Martin}

Department of Civil and Environmental Engineering, Mississippi State University, 501 Hardy Road, 235 Walker Engineering Bldg., Box 9546, Mississippi 39762-9546, USA, jd216@cee.msstate.edu, rac333@cee.msstate.edu, mcanally@cee.msstate.edu, jmartin@cee.msstate.edu

Water resources modelers face the challenge of dealing with numerous uncertainties due to the lack of knowledge of the natural systems, numerical approaches used in modeling (equations, parameters, structures, solutions), and field data collected to set up and evaluate models. Propagation of parameter uncertainty into model results is a relevant topic in environmental hydrology. Uncertainty analyses improve assessment of hydrological modeling. There is a need in modern hydrology of developing and testing uncertainty analysis methods that support hydrological model evaluation. In this research the propagation of model parameter uncertainty into streamflow model results is evaluated. The Hydrological Simulation Program - FORTRAN (HSPF) supported by the US Environmental Protection Agency was evaluated using hydroenvironmental data from the Luxapallila Creek watershed located in Mississippi and Alabama, USA. The uncertainty bounds of model outputs were computed using the Monte Carlo simulation and Harr's point estimation methods. Analysis of parameter uncertainty propagation on streamflow simulations from $12 \mathrm{HSPF}$ parameters was accomplished using 5,000 Monte Carlo random samples and 24 Harr selected points for each selected parameter. The comparison showed that Harr's method could be an appropriate initial indicator of parameter uncertainty propagation on streamflow simulations, particularly for hydrology models with several parameters.

Keywords: HSPF, parameter uncertainty, Monte Carlo simulation, Harr's point estimation method, uncertainty bounds of streamflow simulations sippi State University, 501 Hardy Road, 235 Walker Engineering
Los modeladores de recursos hídricos enfrentan el desafio de
trabajar con diferentes tipos de incertidumbre debido a la
falta de un completo conocimiento de los sistemas naturales,
procesos de modelación, aproximaciones numéricas
(ecuaciones, parámetros, estructuras, soluciones), y datos
de terreno tomados para desarrollar y evaluar modelos.
La propagación de la incertidumbre paramétrica en los
resultados de las simulaciones es un tópico relevante en la
hidrología ambiental. Los análisis de incertidumbre mejoran
la evaluación en el modelamiento hidrológico. Existe una
necesidad en la hidrología moderna de desarrollar y evaluar
métodosdeanálisis deincertidumbreque apoyen la evaluación
de los modelos hidrológicos. En esta investigación se evaluó
la propagación de la incertidumbre de los parámetros de un
modelo en los resultados del flujo simulado. Un programa de
simulación hidrológico HSPF patrocinado por la Agencia
Ambiental de los EE.UU., fue evaluado utilizando datos
hidroambientales de la cuenca de la quebrada Luxapallila
localizada en los estados de Misisipi y Alabama, EE.UU.
Los límites de incertidumbre de las salidas del modelo fueron
calculados utilizando los métodos de simulación Monte Carlo
y el método probabilístico de estimación puntual de Harr. El
análisis de la propagación de la incertidumbre paramétrica
en simulaciones de caudales con HSPF utilizando 12
parámetros fue realizada con 5000 muestras aleatorias de
Monte Carlo y 24 puntos seleccionados de Harr para cada
parámetro evaluado. La comparación mostró que el método
de Harr podría ser un indicador inicial apropiado de la
propagacióndela incertidumbreparamétrica en simulaciones
de caudales, particularmente en modelos hidrológicos con
varios parámetros.

sippi State University, 501 Hardy Road, 235 Walker Engineering
Los modeladores de recursos hídricos enfrentan el desafio de
trabajar con diferentes tipos de incertidumbre debido a la
falta de un completo conocimiento de los sistemas naturales,
procesos de modelación, aproximaciones numéricas
(ecuaciones, parámetros, estructuras, soluciones), y datos
de terreno tomados para desarrollar y evaluar modelos.
La propagación de la incertidumbre paramétrica en los
resultados de las simulaciones es un tópico relevante en la
hidrología ambiental. Los análisis de incertidumbre mejoran
la evaluación en el modelamiento hidrológico. Existe una
necesidad en la hidrología moderna de desarrollar y evaluar
métodosdeanálisis deincertidumbreque apoyen la evaluación
de los modelos hidrológicos. En esta investigación se evaluó
la propagación de la incertidumbre de los parámetros de un
modelo en los resultados del flujo simulado. Un programa de
simulación hidrológico HSPF patrocinado por la Agencia
Ambiental de los EE.UU., fue evaluado utilizando datos
hidroambientales de la cuenca de la quebrada Luxapallila
localizada en los estados de Misisipi y Alabama, EE.UU.
Los límites de incertidumbre de las salidas del modelo fueron
calculados utilizando los métodos de simulación Monte Carlo
y el método probabilístico de estimación puntual de Harr. El
análisis de la propagación de la incertidumbre paramétrica
en simulaciones de caudales con HSPF utilizando 12
parámetros fue realizada con 5000 muestras aleatorias de
Monte Carlo y 24 puntos seleccionados de Harr para cada
parámetro evaluado. La comparación mostró que el método
de Harr podría ser un indicador inicial apropiado de la
propagacióndela incertidumbreparamétrica en simulaciones
de caudales, particularmente en modelos hidrológicos con
varios parámetros.

sippi State University, 501 Hardy Road, 235 Walker Engineering
Los modeladores de recursos hídricos enfrentan el desafio de
trabajar con diferentes tipos de incertidumbre debido a la
falta de un completo conocimiento de los sistemas naturales,
procesos de modelación, aproximaciones numéricas
(ecuaciones, parámetros, estructuras, soluciones), y datos
de terreno tomados para desarrollar y evaluar modelos.
La propagación de la incertidumbre paramétrica en los
resultados de las simulaciones es un tópico relevante en la
hidrología ambiental. Los análisis de incertidumbre mejoran
la evaluación en el modelamiento hidrológico. Existe una
necesidad en la hidrología moderna de desarrollar y evaluar
métodosdeanálisis deincertidumbreque apoyen la evaluación
de los modelos hidrológicos. En esta investigación se evaluó
la propagación de la incertidumbre de los parámetros de un
modelo en los resultados del flujo simulado. Un programa de
simulación hidrológico HSPF patrocinado por la Agencia
Ambiental de los EE.UU., fue evaluado utilizando datos
hidroambientales de la cuenca de la quebrada Luxapallila
localizada en los estados de Misisipi y Alabama, EE.UU.
Los límites de incertidumbre de las salidas del modelo fueron
calculados utilizando los métodos de simulación Monte Carlo
y el método probabilístico de estimación puntual de Harr. El
análisis de la propagación de la incertidumbre paramétrica
en simulaciones de caudales con HSPF utilizando 12
parámetros fue realizada con 5000 muestras aleatorias de
Monte Carlo y 24 puntos seleccionados de Harr para cada
parámetro evaluado. La comparación mostró que el método
de Harr podría ser un indicador inicial apropiado de la
propagacióndela incertidumbreparamétrica en simulaciones
de caudales, particularmente en modelos hidrológicos con
varios parámetros.

ssippi State University, 501 Hardy Road, 235 Walker Engineering
Los modeladores de recursos hídricos enfrentan el desafio de
trabajar con diferentes tipos de incertidumbre debido a la
falta de un completo conocimiento de los sistemas naturales,
procesos de modelación, aproximaciones numéricas
(ecuaciones, parámetros, estructuras, soluciones), y datos
de terreno tomados para desarrollar y evaluar modelos.
La propagación de la incertidumbre paramétrica en los
resultados de las simulaciones es un tópico relevante en la
hidrología ambiental. Los análisis de incertidumbre mejoran
la evaluación en el modelamiento hidrológico. Existe una
necesidad en la hidrología moderna de desarrollar y evaluar
métodosdeanálisis deincertidumbrequeapoyen la evaluación
de los modelos hidrológicos. En esta investigación se evaluó
la propagación de la incertidumbre de los parámetros de un
modelo en los resultados del flujo simulado. Un programa de
simulación hidrológico HSPF patrocinado por la Agencia
Ambiental de los EE.UU., fue evaluado utilizando datos
hidroambientales de la cuenca de la quebrada Luxapallila
localizada en los estados de Misisipi y Alabama, EE.UU.
Los límites de incertidumbre de las salidas del modelo fueron
calculados utilizando los métodos de simulación Monte Carlo
y el método probabilístico de estimación puntual de Harr. El
análisis de la propagación de la incertidumbre paramétrica
en simulaciones de caudales con HSPF utilizando 12
parámetros fue realizada con 5000 muestras aleatorias de
Monte Carlo y 24 puntos seleccionados de Harr para cada
parámetro evaluado. La comparación mostró que el método
de Harr podría ser un indicador inicial apropiado de la
propagacióndela incertidumbreparamétrica en simulaciones
de caudales, particularmente en modelos hidrológicos con
varios parámetros.

sippi State University, 501 Hardy Road, 235 Walker Engineering
Los modeladores de recursos hídricos enfrentan el desafío de
trabajar con diferentes tipos de incertidumbre debido a la
falta de un completo conocimiento de los sistemas naturales,
procesos de modelación, aproximaciones numéricas
(ecuaciones, parámetros, estructuras, soluciones), y datos
de terreno tomados para desarrollar y evaluar modelos.
La propagación de la incertidumbre paramétrica en los
resultados de las simulaciones es un tópico relevante en la
hidrología ambiental. Los análisis de incertidumbre mejoran
la evaluación en el modelamiento hidrológico. Existe una
necesidad en la hidrología moderna de desarrollar y evaluar
métodosdeanálisis deincertidumbrequeapoyen la evaluación
de los modelos hidrológicos. En esta investigación se evaluó
la propagación de la incertidumbre de los parámetros de un
modelo en los resultados del flujo simulado. Un programa de
simulación hidrológico HSPF patrocinado por la Agencia
Ambiental de los EE.UU., fue evaluado utilizando datos
hidroambientales de la cuenca de la quebrada Luxapallila
localizada en los estados de Misisipi y Alabama, EE.UU.
Los límites de incertidumbre de las salidas del modelo fueron
calculados utilizando los métodos de simulación Monte Carlo
y el método probabilístico de estimación puntual de Harr. El
análisis de la propagación de la incertidumbre paramétrica
en simulaciones de caudales con HSPF utilizando 12
parámetros fue realizada con 5000 muestras aleatorias de
Monte Carlo y 24 puntos seleccionados de Harr para cada
parámetro evaluado. La comparación mostró que el método
de Harr podría ser un indicador inicial apropiado de la
propagacióndela incertidumbreparamétrica en simulaciones
de caudales, particularmente en modelos hidrológicos con
varios parámetros.

sippi State University, 501 Hardy Road, 235 Walker Engineering
Los modeladores de recursos hídricos enfrentan el desafío de
trabajar con diferentes tipos de incertidumbre debido a la
falta de un completo conocimiento de los sistemas naturales,
procesos de modelación, aproximaciones numéricas
(ecuaciones, parámetros, estructuras, soluciones), y datos
de terreno tomados para desarrollar y evaluar modelos.
La propagación de la incertidumbre paramétrica en los
resultados de las simulaciones es un tópico relevante en la
hidrología ambiental. Los análisis de incertidumbre mejoran
la evaluación en el modelamiento hidrológico. Existe una
necesidad en la hidrología moderna de desarrollar y evaluar
métodosdeanálisis deincertidumbrequeapoyen la evaluación
de los modelos hidrológicos. En esta investigación se evaluó
la propagación de la incertidumbre de los parámetros de un
modelo en los resultados del flujo simulado. Un programa de
simulación hidrológico HSPF patrocinado por la Agencia
Ambiental de los EE.UU., fue evaluado utilizando datos
hidroambientales de la cuenca de la quebrada Luxapallila
localizada en los estados de Misisipi y Alabama, EE.UU.
Los límites de incertidumbre de las salidas del modelo fueron
calculados utilizando los métodos de simulación Monte Carlo
y el método probabilístico de estimación puntual de Harr. El
análisis de la propagación de la incertidumbre paramétrica
en simulaciones de caudales con HSPF utilizando 12
parámetros fue realizada con 5000 muestras aleatorias de
Monte Carlo y 24 puntos seleccionados de Harr para cada
parámetro evaluado. La comparación mostró que el método
de Harr podría ser un indicador inicial apropiado de la
propagacióndela incertidumbreparamétrica en simulaciones
de caudales, particularmente en modelos hidrológicos con
varios parámetros.

sippi State University, 501 Hardy Road, 235 Walker Engineering
Los modeladores de recursos hídricos enfrentan el desafio de
trabajar con diferentes tipos de incertidumbre debido a la
falta de un completo conocimiento de los sistemas naturales,
procesos de modelación, aproximaciones numéricas
(ecuaciones, parámetros, estructuras, soluciones), y datos
de terreno tomados para desarrollar y evaluar modelos.
La propagación de la incertidumbre paramétrica en los
resultados de las simulaciones es un tópico relevante en la
hidrología ambiental. Los análisis de incertidumbre mejoran
la evaluación en el modelamiento hidrológico. Existe una
necesidad en la hidrología moderna de desarrollar y evaluar
métodosdeanálisis deincertidumbreque apoyen la evaluación
de los modelos hidrológicos. En esta investigación se evaluó
la propagación de la incertidumbre de los parámetros de un
modelo en los resultados del flujo simulado. Un programa de
simulación hidrológico HSPF patrocinado por la Agencia
Ambiental de los EE.UU., fue evaluado utilizando datos
hidroambientales de la cuenca de la quebrada Luxapallila
localizada en los estados de Misisipi y Alabama, EE.UU.
Los límites de incertidumbre de las salidas del modelo fueron
calculados utilizando los métodos de simulación Monte Carlo
y el método probabilístico de estimación puntual de Harr. El
análisis de la propagación de la incertidumbre paramétrica
en simulaciones de caudales con HSPF utilizando 12
parámetros fue realizada con 5000 muestras aleatorias de
Monte Carlo y 24 puntos seleccionados de Harr para cada
parámetro evaluado. La comparación mostró que el método
de Harr podría ser un indicador inicial apropiado de la
propagacióndela incertidumbreparamétrica en simulaciones
de caudales, particularmente en modelos hidrológicos con
varios parámetros.

ssippi State University, 501 Hardy Road, 235 Walker Engineering
Los modeladores de recursos hídricos enfrentan el desafio de
trabajar con diferentes tipos de incertidumbre debido a la
falta de un completo conocimiento de los sistemas naturales,
procesos de modelación, aproximaciones numéricas
(ecuaciones, parámetros, estructuras, soluciones), y datos
de terreno tomados para desarrollar y evaluar modelos.
La propagación de la incertidumbre paramétrica en los
resultados de las simulaciones es un tópico relevante en la
hidrología ambiental. Los análisis de incertidumbre mejoran
la evaluación en el modelamiento hidrológico. Existe una
necesidad en la hidrología moderna de desarrollar y evaluar
métodosdeanálisis deincertidumbrequeapoyen la evaluación
de los modelos hidrológicos. En esta investigación se evaluó
la propagación de la incertidumbre de los parámetros de un
modelo en los resultados del flujo simulado. Un programa de
simulación hidrológico HSPF patrocinado por la Agencia
Ambiental de los EE.UU., fue evaluado utilizando datos
hidroambientales de la cuenca de la quebrada Luxapallila
localizada en los estados de Misisipi y Alabama, EE.UU.
Los límites de incertidumbre de las salidas del modelo fueron
calculados utilizando los métodos de simulación Monte Carlo
y el método probabilístico de estimación puntual de Harr. El
análisis de la propagación de la incertidumbre paramétrica
en simulaciones de caudales con HSPF utilizando 12
parámetros fue realizada con 5000 muestras aleatorias de
Monte Carlo y 24 puntos seleccionados de Harr para cada
parámetro evaluado. La comparación mostró que el método
de Harr podría ser un indicador inicial apropiado de la
propagacióndela incertidumbreparamétrica en simulaciones
de caudales, particularmente en modelos hidrológicos con
varios parámetros.

sippi State University, 501 Hardy Road, 235 Walker Engineering
Los modeladores de recursos hídricos enfrentan el desafío de
trabajar con diferentes tipos de incertidumbre debido a la
falta de un completo conocimiento de los sistemas naturales,
procesos de modelación, aproximaciones numéricas
(ecuaciones, parámetros, estructuras, soluciones), y datos
de terreno tomados para desarrollar y evaluar modelos.
La propagación de la incertidumbre paramétrica en los
resultados de las simulaciones es un tópico relevante en la
hidrología ambiental. Los análisis de incertidumbre mejoran
la evaluación en el modelamiento hidrológico. Existe una
necesidad en la hidrología moderna de desarrollar y evaluar
métodosdeanálisis deincertidumbrequeapoyen la evaluación
de los modelos hidrológicos. En esta investigación se evaluó
la propagación de la incertidumbre de los parámetros de un
modelo en los resultados del flujo simulado. Un programa de
simulación hidrológico HSPF patrocinado por la Agencia
Ambiental de los EE.UU., fue evaluado utilizando datos
hidroambientales de la cuenca de la quebrada Luxapallila
localizada en los estados de Misisipi y Alabama, EE.UU.
Los límites de incertidumbre de las salidas del modelo fueron
calculados utilizando los métodos de simulación Monte Carlo
y el método probabilístico de estimación puntual de Harr. El
análisis de la propagación de la incertidumbre paramétrica
en simulaciones de caudales con HSPF utilizando 12
parámetros fue realizada con 5000 muestras aleatorias de
Monte Carlo y 24 puntos seleccionados de Harr para cada
parámetro evaluado. La comparación mostró que el método
de Harr podría ser un indicador inicial apropiado de la
propagacióndela incertidumbreparamétrica en simulaciones
de caudales, particularmente en modelos hidrológicos con
varios parámetros.

sippi State University, 501 Hardy Road, 235 Walker Engineering
Los modeladores de recursos hídricos enfrentan el desafío de
trabajar con diferentes tipos de incertidumbre debido a la
falta de un completo conocimiento de los sistemas naturales,
procesos de modelación, aproximaciones numéricas
(ecuaciones, parámetros, estructuras, soluciones), y datos
de terreno tomados para desarrollar y evaluar modelos.
La propagación de la incertidumbre paramétrica en los
resultados de las simulaciones es un tópico relevante en la
hidrología ambiental. Los análisis de incertidumbre mejoran
la evaluación en el modelamiento hidrológico. Existe una
necesidad en la hidrología moderna de desarrollar y evaluar
métodosdeanálisis deincertidumbrequeapoyen la evaluación
de los modelos hidrológicos. En esta investigación se evaluó
la propagación de la incertidumbre de los parámetros de un
modelo en los resultados del flujo simulado. Un programa de
simulación hidrológico HSPF patrocinado por la Agencia
Ambiental de los EE.UU., fue evaluado utilizando datos
hidroambientales de la cuenca de la quebrada Luxapallila
localizada en los estados de Misisipi y Alabama, EE.UU.
Los límites de incertidumbre de las salidas del modelo fueron
calculados utilizando los métodos de simulación Monte Carlo
y el método probabilístico de estimación puntual de Harr. El
análisis de la propagación de la incertidumbre paramétrica
en simulaciones de caudales con HSPF utilizando 12
parámetros fue realizada con 5000 muestras aleatorias de
Monte Carlo y 24 puntos seleccionados de Harr para cada
parámetro evaluado. La comparación mostró que el método
de Harr podría ser un indicador inicial apropiado de la
propagacióndela incertidumbreparamétrica en simulaciones
de caudales, particularmente en modelos hidrológicos con
varios parámetros.

sippi State University, 501 Hardy Road, 235 Walker Engineering
Los modeladores de recursos hídricos enfrentan el desafio de
trabajar con diferentes tipos de incertidumbre debido a la
falta de un completo conocimiento de los sistemas naturales,
procesos de modelación, aproximaciones numéricas
(ecuaciones, parámetros, estructuras, soluciones), y datos
de terreno tomados para desarrollar y evaluar modelos.
La propagación de la incertidumbre paramétrica en los
resultados de las simulaciones es un tópico relevante en la
hidrología ambiental. Los análisis de incertidumbre mejoran
la evaluación en el modelamiento hidrológico. Existe una
necesidad en la hidrología moderna de desarrollar y evaluar
métodosdeanálisis deincertidumbreque apoyen la evaluación
de los modelos hidrológicos. En esta investigación se evaluó
la propagación de la incertidumbre de los parámetros de un
modelo en los resultados del flujo simulado. Un programa de
simulación hidrológico HSPF patrocinado por la Agencia
Ambiental de los EE.UU., fue evaluado utilizando datos
hidroambientales de la cuenca de la quebrada Luxapallila
localizada en los estados de Misisipi y Alabama, EE.UU.
Los límites de incertidumbre de las salidas del modelo fueron
calculados utilizando los métodos de simulación Monte Carlo
y el método probabilístico de estimación puntual de Harr. El
análisis de la propagación de la incertidumbre paramétrica
en simulaciones de caudales con HSPF utilizando 12
parámetros fue realizada con 5000 muestras aleatorias de
Monte Carlo y 24 puntos seleccionados de Harr para cada
parámetro evaluado. La comparación mostró que el método
de Harr podría ser un indicador inicial apropiado de la
propagacióndela incertidumbreparamétrica en simulaciones
de caudales, particularmente en modelos hidrológicos con
varios parámetros.

sippi State University, 501 Hardy Road, 235 Walker Engineering
Los modeladores de recursos hídricos enfrentan el desafío de
trabajar con diferentes tipos de incertidumbre debido a la
falta de un completo conocimiento de los sistemas naturales,
procesos de modelación, aproximaciones numéricas
(ecuaciones, parámetros, estructuras, soluciones), y datos
de terreno tomados para desarrollar y evaluar modelos.
La propagación de la incertidumbre paramétrica en los
resultados de las simulaciones es un tópico relevante en la
hidrología ambiental. Los análisis de incertidumbre mejoran
la evaluación en el modelamiento hidrológico. Existe una
necesidad en la hidrología moderna de desarrollar y evaluar
métodosdeanálisis deincertidumbrequeapoyen la evaluación
de los modelos hidrológicos. En esta investigación se evaluó
la propagación de la incertidumbre de los parámetros de un
modelo en los resultados del flujo simulado. Un programa de
simulación hidrológico HSPF patrocinado por la Agencia
Ambiental de los EE.UU., fue evaluado utilizando datos
hidroambientales de la cuenca de la quebrada Luxapallila
localizada en los estados de Misisipi y Alabama, EE.UU.
Los límites de incertidumbre de las salidas del modelo fueron
calculados utilizando los métodos de simulación Monte Carlo
y el método probabilístico de estimación puntual de Harr. El
análisis de la propagación de la incertidumbre paramétrica
en simulaciones de caudales con HSPF utilizando 12
parámetros fue realizada con 5000 muestras aleatorias de
Monte Carlo y 24 puntos seleccionados de Harr para cada
parámetro evaluado. La comparación mostró que el método
de Harr podría ser un indicador inicial apropiado de la
propagacióndela incertidumbreparamétrica en simulaciones
de caudales, particularmente en modelos hidrológicos con
varios parámetros.

sippi State University, 501 Hardy Road, 235 Walker Engineering
Los modeladores de recursos hídricos enfrentan el desafío de
trabajar con diferentes tipos de incertidumbre debido a la
falta de un completo conocimiento de los sistemas naturales,
procesos de modelación, aproximaciones numéricas
(ecuaciones, parámetros, estructuras, soluciones), y datos
de terreno tomados para desarrollar y evaluar modelos.
La propagación de la incertidumbre paramétrica en los
resultados de las simulaciones es un tópico relevante en la
hidrología ambiental. Los análisis de incertidumbre mejoran
la evaluación en el modelamiento hidrológico. Existe una
necesidad en la hidrología moderna de desarrollar y evaluar
métodosdeanálisis deincertidumbrequeapoyen la evaluación
de los modelos hidrológicos. En esta investigación se evaluó
la propagación de la incertidumbre de los parámetros de un
modelo en los resultados del flujo simulado. Un programa de
simulación hidrológico HSPF patrocinado por la Agencia
Ambiental de los EE.UU., fue evaluado utilizando datos
hidroambientales de la cuenca de la quebrada Luxapallila
localizada en los estados de Misisipi y Alabama, EE.UU.
Los límites de incertidumbre de las salidas del modelo fueron
calculados utilizando los métodos de simulación Monte Carlo
y el método probabilístico de estimación puntual de Harr. El
análisis de la propagación de la incertidumbre paramétrica
en simulaciones de caudales con HSPF utilizando 12
parámetros fue realizada con 5000 muestras aleatorias de
Monte Carlo y 24 puntos seleccionados de Harr para cada
parámetro evaluado. La comparación mostró que el método
de Harr podría ser un indicador inicial apropiado de la
propagacióndela incertidumbreparamétrica en simulaciones
de caudales, particularmente en modelos hidrológicos con
varios parámetros.

sippi State University, 501 Hardy Road, 235 Walker Engineering
Los modeladores de recursos hídricos enfrentan el desafío de
trabajar con diferentes tipos de incertidumbre debido a la
falta de un completo conocimiento de los sistemas naturales,
procesos de modelación, aproximaciones numéricas
(ecuaciones, parámetros, estructuras, soluciones), y datos
de terreno tomados para desarrollar y evaluar modelos.
La propagación de la incertidumbre paramétrica en los
resultados de las simulaciones es un tópico relevante en la
hidrología ambiental. Los análisis de incertidumbre mejoran
la evaluación en el modelamiento hidrológico. Existe una
necesidad en la hidrología moderna de desarrollar y evaluar
métodosdeanálisis deincertidumbrequeapoyen la evaluación
de los modelos hidrológicos. En esta investigación se evaluó
la propagación de la incertidumbre de los parámetros de un
modelo en los resultados del flujo simulado. Un programa de
simulación hidrológico HSPF patrocinado por la Agencia
Ambiental de los EE.UU., fue evaluado utilizando datos
hidroambientales de la cuenca de la quebrada Luxapallila
localizada en los estados de Misisipi y Alabama, EE.UU.
Los límites de incertidumbre de las salidas del modelo fueron
calculados utilizando los métodos de simulación Monte Carlo
y el método probabilístico de estimación puntual de Harr. El
análisis de la propagación de la incertidumbre paramétrica
en simulaciones de caudales con HSPF utilizando 12
parámetros fue realizada con 5000 muestras aleatorias de
Monte Carlo y 24 puntos seleccionados de Harr para cada
parámetro evaluado. La comparación mostró que el método
de Harr podría ser un indicador inicial apropiado de la
propagacióndela incertidumbreparamétrica en simulaciones
de caudales, particularmente en modelos hidrológicos con
varios parámetros.

sippi State University, 501 Hardy Road, 235 Walker Engineering
Los modeladores de recursos hídricos enfrentan el desafío de
trabajar con diferentes tipos de incertidumbre debido a la
falta de un completo conocimiento de los sistemas naturales,
procesos de modelación, aproximaciones numéricas
(ecuaciones, parámetros, estructuras, soluciones), y datos
de terreno tomados para desarrollar y evaluar modelos.
La propagación de la incertidumbre paramétrica en los
resultados de las simulaciones es un tópico relevante en la
hidrología ambiental. Los análisis de incertidumbre mejoran
la evaluación en el modelamiento hidrológico. Existe una
necesidad en la hidrología moderna de desarrollar y evaluar
métodosdeanálisis deincertidumbrequeapoyen la evaluación
de los modelos hidrológicos. En esta investigación se evaluó
la propagación de la incertidumbre de los parámetros de un
modelo en los resultados del flujo simulado. Un programa de
simulación hidrológico HSPF patrocinado por la Agencia
Ambiental de los EE.UU., fue evaluado utilizando datos
hidroambientales de la cuenca de la quebrada Luxapallila
localizada en los estados de Misisipi y Alabama, EE.UU.
Los límites de incertidumbre de las salidas del modelo fueron
calculados utilizando los métodos de simulación Monte Carlo
y el método probabilístico de estimación puntual de Harr. El
análisis de la propagación de la incertidumbre paramétrica
en simulaciones de caudales con HSPF utilizando 12
parámetros fue realizada con 5000 muestras aleatorias de
Monte Carlo y 24 puntos seleccionados de Harr para cada
parámetro evaluado. La comparación mostró que el método
de Harr podría ser un indicador inicial apropiado de la
propagacióndela incertidumbreparamétrica en simulaciones
de caudales, particularmente en modelos hidrológicos con
varios parámetros.

sippi State University, 501 Hardy Road, 235 Walker Engineering
Los modeladores de recursos hídricos enfrentan el desafío de
trabajar con diferentes tipos de incertidumbre debido a la
falta de un completo conocimiento de los sistemas naturales,
procesos de modelación, aproximaciones numéricas
(ecuaciones, parámetros, estructuras, soluciones), y datos
de terreno tomados para desarrollar y evaluar modelos.
La propagación de la incertidumbre paramétrica en los
resultados de las simulaciones es un tópico relevante en la
hidrología ambiental. Los análisis de incertidumbre mejoran
la evaluación en el modelamiento hidrológico. Existe una
necesidad en la hidrología moderna de desarrollar y evaluar
métodosdeanálisis deincertidumbrequeapoyen la evaluación
de los modelos hidrológicos. En esta investigación se evaluó
la propagación de la incertidumbre de los parámetros de un
modelo en los resultados del flujo simulado. Un programa de
simulación hidrológico HSPF patrocinado por la Agencia
Ambiental de los EE.UU., fue evaluado utilizando datos
hidroambientales de la cuenca de la quebrada Luxapallila
localizada en los estados de Misisipi y Alabama, EE.UU.
Los límites de incertidumbre de las salidas del modelo fueron
calculados utilizando los métodos de simulación Monte Carlo
y el método probabilístico de estimación puntual de Harr. El
análisis de la propagación de la incertidumbre paramétrica
en simulaciones de caudales con HSPF utilizando 12
parámetros fue realizada con 5000 muestras aleatorias de
Monte Carlo y 24 puntos seleccionados de Harr para cada
parámetro evaluado. La comparación mostró que el método
de Harr podría ser un indicador inicial apropiado de la
propagacióndela incertidumbreparamétrica en simulaciones
de caudales, particularmente en modelos hidrológicos con
varios parámetros.

sippi State University, 501 Hardy Road, 235 Walker Engineering
Los modeladedu, rac333@cee.msstate.edu, mcanally@cee.msstate.edu,
trabajar con diferentes tipos de incertidumbre debido a la
falta de un completo conocimiento de los sistemas naturales,
procesos de modelación, aproximaciones numéricas
(ecuaciones, parámetros, estructuras, soluciones), y datos
de terreno tomados para desarrollar y evaluar modelos.
La propagación de la incertidumbre paramétrica en los
resultados de las simulaciones es un tópico relevante en la
hidrología ambiental. Los análisis de incertidumbre mejoran
la evaluación en el modelamiento hidrológico. Existe una
necesidad en la hidrología moderna de desarrollar y evaluar
métodosdeanálisis deincertidumbrequeapoyen la evaluación
de los modelos hidrológicos. En esta investigación se evaluó
la propagación de la incertidumbre de los parámetros de un
modelo en los resultados del flujo simulado. Un programa de
simulación hidrológico HSPF patrocinado por la Agencia
Ambiental de los EE.UU., fue evaluado utilizando datos
hidroambientales de la cuenca de la quebrada Luxapallila
localizada en los estados de Misisipi y Alabama, EE.UU.
Los límites de incertidumbre de las salidas del modelo fueron
calculados utilizando los métodos de simulación Monte Carlo
y el método probabilistico de estimación puntual de Harr. El
análisis de la propagación de la incertidumbre paramétrica
en simulaciones de caudales con HSPF utilizando 12
parámetros fue realizada con 5000 muestras aleatorias de
Monte Carlo y 24 puntos seleccionados de Harr para cada
parámetro evaluado. La comparación mostró que el método
de Harr podría ser un indicador inicial apropiado de la
propagacióndelaincertidumbreparamétrica en simulaciones
de caudales, particularmente en modelos hidrológicos con
varios parámetros.

sippi State University, 501 Hardy Road, 235 Walker Engineering
Los modeladores de recursos hídricos enfrentan el desafío de
trabajar con diferentes tipos de incertidumbre debido a la
falta de un completo conocimiento de los sistemas naturales,
procesos de modelación, aproximaciones numéricas
(ecuaciones, parámetros, estructuras, soluciones), y datos
de terreno tomados para desarrollar y evaluar modelos.
La propagación de la incertidumbre paramétrica en los
resultados de las simulaciones es un tópico relevante en la
hidrología ambiental. Los análisis de incertidumbre mejoran
la evaluación en el modelamiento hidrológico. Existe una
necesidad en la hidrología moderna de desarrollar y evaluar
métodosdeanálisis deincertidumbrequeapoyen la evaluación
de los modelos hidrológicos. En esta investigación se evaluó
la propagación de la incertidumbre de los parámetros de un
modelo en los resultados del flujo simulado. Un programa de
simulación hidrológico HSPF patrocinado por la Agencia
Ambiental de los EE.UU., fue evaluado utilizando datos
hidroambientales de la cuenca de la quebrada Luxapallila
localizada en los estados de Misisipi y Alabama, EE.UU.
Los límites de incertidumbre de las salidas del modelo fueron
calculados utilizando los métodos de simulación Monte Carlo
y el método probabilístico de estimación puntual de Harr. El
análisis de la propagación de la incertidumbre paramétrica
en simulaciones de caudales con HSPF utilizando 12
parámetros fue realizada con 5000 muestras aleatorias de
Monte Carlo y 24 puntos seleccionados de Harr para cada
parámetro evaluado. La comparación mostró que el método
de Harr podría ser un indicador inicial apropiado de la
propagacióndela incertidumbreparamétrica en simulaciones
de caudales, particularmente en modelos hidrológicos con
varios parámetros.

sippi State University, 501 Hardy Road, 235 Walker Engineering
Los modeladores de recursos hídricos enfrentan el desafío de
trabajar con diferentes tipos de incertidumbre debido a la
falta de un completo conocimiento de los sistemas naturales,
procesos de modelación, aproximaciones numéricas
(ecuaciones, parámetros, estructuras, soluciones), y datos
de terreno tomados para desarrollar y evaluar modelos.
La propagación de la incertidumbre paramétrica en los
resultados de las simulaciones es un tópico relevante en la
hidrología ambiental. Los análisis de incertidumbre mejoran
la evaluación en el modelamiento hidrológico. Existe una
necesidad en la hidrología moderna de desarrollar y evaluar
métodosdeanálisis deincertidumbrequeapoyen la evaluación
de los modelos hidrológicos. En esta investigación se evaluó
la propagación de la incertidumbre de los parámetros de un
modelo en los resultados del flujo simulado. Un programa de
simulación hidrológico HSPF patrocinado por la Agencia
Ambiental de los EE.UU., fue evaluado utilizando datos
hidroambientales de la cuenca de la quebrada Luxapallila
localizada en los estados de Misisipi y Alabama, EE.UU.
Los límites de incertidumbre de las salidas del modelo fueron
calculados utilizando los métodos de simulación Monte Carlo
y el método probabilístico de estimación puntual de Harr. El
análisis de la propagación de la incertidumbre paramétrica
en simulaciones de caudales con HSPF utilizando 12
parámetros fue realizada con 5000 muestras aleatorias de
Monte Carlo y 24 puntos seleccionados de Harr para cada
parámetro evaluado. La comparación mostró que el método
de Harr podría ser un indicador inicial apropiado de la
propagacióndela incertidumbreparamétrica en simulaciones
de caudales, particularmente en modelos hidrológicos con
varios parámetros.

sippi State University, 501 Hardy Road, 235 Walker Engineering
Los modeladores de recursos hídricos enfrentan el desafio de
trabajar con diferentes tipos de incertidumbre debido a la
falta de un completo conocimiento de los sistemas naturales,
procesos de modelación, aproximaciones numéricas
(ecuaciones, parámetros, estructuras, soluciones), y datos
de terreno tomados para desarrollar y evaluar modelos.
La propagación de la incertidumbre paramétrica en los
resultados de las simulaciones es un tópico relevante en la
hidrología ambiental. Los análisis de incertidumbre mejoran
la evaluación en el modelamiento hidrológico. Existe una
necesidad en la hidrología moderna de desarrollar y evaluar
métodosdeanálisis deincertidumbreque apoyen la evaluación
de los modelos hidrológicos. En esta investigación se evaluó
la propagación de la incertidumbre de los parámetros de un
modelo en los resultados del flujo simulado. Un programa de
simulación hidrológico HSPF patrocinado por la Agencia
Ambiental de los EE.UU., fue evaluado utilizando datos
hidroambientales de la cuenca de la quebrada Luxapallila
localizada en los estados de Misisipi y Alabama, EE.UU.
Los límites de incertidumbre de las salidas del modelo fueron
calculados utilizando los métodos de simulación Monte Carlo
y el método probabilístico de estimación puntual de Harr. El
análisis de la propagación de la incertidumbre paramétrica
en simulaciones de caudales con HSPF utilizando 12
parámetros fue realizada con 5000 muestras aleatorias de
Monte Carlo y 24 puntos seleccionados de Harr para cada
parámetro evaluado. La comparación mostró que el método
de Harr podría ser un indicador inicial apropiado de la
propagacióndela incertidumbreparamétrica en simulaciones
de caudales, particularmente en modelos hidrológicos con
varios parámetros.

sippi State University, 501 Hardy Road, 235 Walker Engineering
Los modeladores de recursos hídricos enfrentan el desafío de
trabajar con diferentes tipos de incertidumbre debido a la
falta de un completo conocimiento de los sistemas naturales,
procesos de modelación, aproximaciones numéricas
(ecuaciones, parámetros, estructuras, soluciones), y datos
de terreno tomados para desarrollar y evaluar modelos.
La propagación de la incertidumbre paramétrica en los
resultados de las simulaciones es un tópico relevante en la
hidrología ambiental. Los análisis de incertidumbre mejoran
la evaluación en el modelamiento hidrológico. Existe una
necesidad en la hidrología moderna de desarrollar y evaluar
métodosdeanálisis deincertidumbrequeapoyen la evaluación
de los modelos hidrológicos. En esta investigación se evaluó
la propagación de la incertidumbre de los parámetros de un
modelo en los resultados del flujo simulado. Un programa de
simulación hidrológico HSPF patrocinado por la Agencia
Ambiental de los EE.UU., fue evaluado utilizando datos
hidroambientales de la cuenca de la quebrada Luxapallila
localizada en los estados de Misisipi y Alabama, EE.UU.
Los límites de incertidumbre de las salidas del modelo fueron
calculados utilizando los métodos de simulación Monte Carlo
y el método probabilístico de estimación puntual de Harr. El
análisis de la propagación de la incertidumbre paramétrica
en simulaciones de caudales con HSPF utilizando 12
parámetros fue realizada con 5000 muestras aleatorias de
Monte Carlo y 24 puntos seleccionados de Harr para cada
parámetro evaluado. La comparación mostró que el método
de Harr podría ser un indicador inicial apropiado de la
propagacióndela incertidumbreparamétrica en simulaciones
de caudales, particularmente en modelos hidrológicos con
varios parámetros.

sippi State University, 501 Hardy Road, 235 Walker Engineering
Los modeladores de recursos hídricos enfrentan el desafio de
trabajar con diferentes tipos de incertidumbre debido a la
falta de un completo conocimiento de los sistemas naturales,
procesos de modelación, aproximaciones numéricas
(ecuaciones, parámetros, estructuras, soluciones), y datos
de terreno tomados para desarrollar y evaluar modelos.
La propagación de la incertidumbre paramétrica en los
resultados de las simulaciones es un tópico relevante en la
hidrología ambiental. Los análisis de incertidumbre mejoran
la evaluación en el modelamiento hidrológico. Existe una
necesidad en la hidrología moderna de desarrollar y evaluar
métodosdeanálisis deincertidumbreque apoyen la evaluación
de los modelos hidrológicos. En esta investigación se evaluó
la propagación de la incertidumbre de los parámetros de un
modelo en los resultados del flujo simulado. Un programa de
simulación hidrológico HSPF patrocinado por la Agencia
Ambiental de los EE.UU., fue evaluado utilizando datos
hidroambientales de la cuenca de la quebrada Luxapallila
localizada en los estados de Misisipi y Alabama, EE.UU.
Los límites de incertidumbre de las salidas del modelo fueron
calculados utilizando los métodos de simulación Monte Carlo
y el método probabilístico de estimación puntual de Harr. El
análisis de la propagación de la incertidumbre paramétrica
en simulaciones de caudales con HSPF utilizando 12
parámetros fue realizada con 5000 muestras aleatorias de
Monte Carlo y 24 puntos seleccionados de Harr para cada
parámetro evaluado. La comparación mostró que el método
de Harr podría ser un indicador inicial apropiado de la
propagacióndela incertidumbreparamétrica en simulaciones
de caudales, particularmente en modelos hidrológicos con
varios parámetros.

sippi State University, 501 Hardy Road, 235 Walker Engineering
Los modeladores de recursos hídricos enfrentan el desafío de
trabajar con diferentes tipos de incertidumbre debido a la
falta de un completo conocimiento de los sistemas naturales,
procesos de modelación, aproximaciones numéricas
(ecuaciones, parámetros, estructuras, soluciones), y datos
de terreno tomados para desarrollar y evaluar modelos.
La propagación de la incertidumbre paramétrica en los
resultados de las simulaciones es un tópico relevante en la
hidrología ambiental. Los análisis de incertidumbre mejoran
la evaluación en el modelamiento hidrológico. Existe una
necesidad en la hidrología moderna de desarrollar y evaluar
métodosdeanálisis deincertidumbrequeapoyen la evaluación
de los modelos hidrológicos. En esta investigación se evaluó
la propagación de la incertidumbre de los parámetros de un
modelo en los resultados del flujo simulado. Un programa de
simulación hidrológico HSPF patrocinado por la Agencia
Ambiental de los EE.UU., fue evaluado utilizando datos
hidroambientales de la cuenca de la quebrada Luxapallila
localizada en los estados de Misisipi y Alabama, EE.UU.
Los límites de incertidumbre de las salidas del modelo fueron
calculados utilizando los métodos de simulación Monte Carlo
y el método probabilístico de estimación puntual de Harr. El
análisis de la propagación de la incertidumbre paramétrica
en simulaciones de caudales con HSPF utilizando 12
parámetros fue realizada con 5000 muestras aleatorias de
Monte Carlo y 24 puntos seleccionados de Harr para cada
parámetro evaluado. La comparación mostró que el método
de Harr podría ser un indicador inicial apropiado de la
propagacióndela incertidumbreparamétrica en simulaciones
de caudales, particularmente en modelos hidrológicos con
varios parámetros.

sippi State University, 501 Hardy Road, 235 Walker Engineering
Los modeladores de recursos hídricos enfrentan el desafio de
trabajar con diferentes tipos de incertidumbre debido a la
falta de un completo conocimiento de los sistemas naturales,
procesos de modelación, aproximaciones numéricas
(ecuaciones, parámetros, estructuras, soluciones), y datos
de terreno tomados para desarrollar y evaluar modelos.
La propagación de la incertidumbre paramétrica en los
resultados de las simulaciones es un tópico relevante en la
hidrología ambiental. Los análisis de incertidumbre mejoran
la evaluación en el modelamiento hidrológico. Existe una
necesidad en la hidrología moderna de desarrollar y evaluar
métodosdeanálisis deincertidumbreque apoyen la evaluación
de los modelos hidrológicos. En esta investigación se evaluó
la propagación de la incertidumbre de los parámetros de un
modelo en los resultados del flujo simulado. Un programa de
simulación hidrológico HSPF patrocinado por la Agencia
Ambiental de los EE.UU., fue evaluado utilizando datos
hidroambientales de la cuenca de la quebrada Luxapallila
localizada en los estados de Misisipi y Alabama, EE.UU.
Los límites de incertidumbre de las salidas del modelo fueron
calculados utilizando los métodos de simulación Monte Carlo
y el método probabilístico de estimación puntual de Harr. El
análisis de la propagación de la incertidumbre paramétrica
en simulaciones de caudales con HSPF utilizando 12
parámetros fue realizada con 5000 muestras aleatorias de
Monte Carlo y 24 puntos seleccionados de Harr para cada
parámetro evaluado. La comparación mostró que el método
de Harr podría ser un indicador inicial apropiado de la
propagacióndela incertidumbreparamétrica en simulaciones
de caudales, particularmente en modelos hidrológicos con
varios parámetros.

sippi State University, 501 Hardy Road, 235 Walker Engineering
Los modeladores de recursos hídricos enfrentan el desafío de
trabajar con diferentes tipos de incertidumbre debido a la
falta de un completo conocimiento de los sistemas naturales,
procesos de modelación, aproximaciones numéricas
(ecuaciones, parámetros, estructuras, soluciones), y datos
de terreno tomados para desarrollar y evaluar modelos.
La propagación de la incertidumbre paramétrica en los
resultados de las simulaciones es un tópico relevante en la
hidrología ambiental. Los análisis de incertidumbre mejoran
la evaluación en el modelamiento hidrológico. Existe una
necesidad en la hidrología moderna de desarrollar y evaluar
métodosdeanálisis deincertidumbrequeapoyen la evaluación
de los modelos hidrológicos. En esta investigación se evaluó
la propagación de la incertidumbre de los parámetros de un
modelo en los resultados del flujo simulado. Un programa de
simulación hidrológico HSPF patrocinado por la Agencia
Ambiental de los EE.UU., fue evaluado utilizando datos
hidroambientales de la cuenca de la quebrada Luxapallila
localizada en los estados de Misisipi y Alabama, EE.UU.
Los límites de incertidumbre de las salidas del modelo fueron
calculados utilizando los métodos de simulación Monte Carlo
y el método probabilístico de estimación puntual de Harr. El
análisis de la propagación de la incertidumbre paramétrica
en simulaciones de caudales con HSPF utilizando 12
parámetros fue realizada con 5000 muestras aleatorias de
Monte Carlo y 24 puntos seleccionados de Harr para cada
parámetro evaluado. La comparación mostró que el método
de Harr podría ser un indicador inicial apropiado de la
propagacióndela incertidumbreparamétrica en simulaciones
de caudales, particularmente en modelos hidrológicos con
varios parámetros.

sippi State University, 501 Hardy Road, 235 Walker Engineering
Los modeladores de recursos hídricos enfrentan el desafío de
trabajar con diferentes tipos de incertidumbre debido a la
falta de un completo conocimiento de los sistemas naturales,
procesos de modelación, aproximaciones numéricas
(ecuaciones, parámetros, estructuras, soluciones), y datos
de terreno tomados para desarrollar y evaluar modelos.
La propagación de la incertidumbre paramétrica en los
resultados de las simulaciones es un tópico relevante en la
hidrología ambiental. Los análisis de incertidumbre mejoran
la evaluación en el modelamiento hidrológico. Existe una
necesidad en la hidrología moderna de desarrollar y evaluar
métodosdeanálisis deincertidumbrequeapoyen la evaluación
de los modelos hidrológicos. En esta investigación se evaluó
la propagación de la incertidumbre de los parámetros de un
modelo en los resultados del flujo simulado. Un programa de
simulación hidrológico HSPF patrocinado por la Agencia
Ambiental de los EE.UU., fue evaluado utilizando datos
hidroambientales de la cuenca de la quebrada Luxapallila
localizada en los estados de Misisipi y Alabama, EE.UU.
Los límites de incertidumbre de las salidas del modelo fueron
calculados utilizando los métodos de simulación Monte Carlo
y el método probabilístico de estimación puntual de Harr. El
análisis de la propagación de la incertidumbre paramétrica
en simulaciones de caudales con HSPF utilizando 12
parámetros fue realizada con 5000 muestras aleatorias de
Monte Carlo y 24 puntos seleccionados de Harr para cada
parámetro evaluado. La comparación mostró que el método
de Harr podría ser un indicador inicial apropiado de la
propagacióndela incertidumbreparamétrica en simulaciones
de caudales, particularmente en modelos hidrológicos con
varios parámetros.

sippi State University, 501 Hardy Road, 235 Walker Engineering
Los modeladores de recursos hídricos enfrentan el desafío de
trabajar con diferentes tipos de incertidumbre debido a la
falta de un completo conocimiento de los sistemas naturales,
procesos de modelación, aproximaciones numéricas
(ecuaciones, parámetros, estructuras, soluciones), y datos
de terreno tomados para desarrollar y evaluar modelos.
La propagación de la incertidumbre paramétrica en los
resultados de las simulaciones es un tópico relevante en la
hidrología ambiental. Los análisis de incertidumbre mejoran
la evaluación en el modelamiento hidrológico. Existe una
necesidad en la hidrología moderna de desarrollar y evaluar
métodosdeanálisis deincertidumbrequeapoyen la evaluación
de los modelos hidrológicos. En esta investigación se evaluó
la propagación de la incertidumbre de los parámetros de un
modelo en los resultados del flujo simulado. Un programa de
simulación hidrológico HSPF patrocinado por la Agencia
Ambiental de los EE.UU., fue evaluado utilizando datos
hidroambientales de la cuenca de la quebrada Luxapallila
localizada en los estados de Misisipi y Alabama, EE.UU.
Los límites de incertidumbre de las salidas del modelo fueron
calculados utilizando los métodos de simulación Monte Carlo
y el método probabilístico de estimación puntual de Harr. El
análisis de la propagación de la incertidumbre paramétrica
en simulaciones de caudales con HSPF utilizando 12
parámetros fue realizada con 5000 muestras aleatorias de
Monte Carlo y 24 puntos seleccionados de Harr para cada
parámetro evaluado. La comparación mostró que el método
de Harr podría ser un indicador inicial apropiado de la
propagacióndela incertidumbreparamétrica en simulaciones
de caudales, particularmente en modelos hidrológicos con
varios parámetros.

sippi State University, 501 Hardy Road, 235 Walker Engineering
Los modeladores de recursos hídricos enfrentan el desafío de
trabajar con diferentes tipos de incertidumbre debido a la
falta de un completo conocimiento de los sistemas naturales,
procesos de modelación, aproximaciones numéricas
(ecuaciones, parámetros, estructuras, soluciones), y datos
de terreno tomados para desarrollar y evaluar modelos.
La propagación de la incertidumbre paramétrica en los
resultados de las simulaciones es un tópico relevante en la
hidrología ambiental. Los análisis de incertidumbre mejoran
la evaluación en el modelamiento hidrológico. Existe una
necesidad en la hidrología moderna de desarrollar y evaluar
métodosdeanálisis deincertidumbrequeapoyen la evaluación
de los modelos hidrológicos. En esta investigación se evaluó
la propagación de la incertidumbre de los parámetros de un
modelo en los resultados del flujo simulado. Un programa de
simulación hidrológico HSPF patrocinado por la Agencia
Ambiental de los EE.UU., fue evaluado utilizando datos
hidroambientales de la cuenca de la quebrada Luxapallila
localizada en los estados de Misisipi y Alabama, EE.UU.
Los límites de incertidumbre de las salidas del modelo fueron
calculados utilizando los métodos de simulación Monte Carlo
y el método probabilístico de estimación puntual de Harr. El
análisis de la propagación de la incertidumbre paramétrica
en simulaciones de caudales con HSPF utilizando 12
parámetros fue realizada con 5000 muestras aleatorias de
Monte Carlo y 24 puntos seleccionados de Harr para cada
parámetro evaluado. La comparación mostró que el método
de Harr podría ser un indicador inicial apropiado de la
propagacióndela incertidumbreparamétrica en simulaciones
de caudales, particularmente en modelos hidrológicos con
varios parámetros.

sippi State University, 501 Hardy Road, 235 Walker Engineering
Los modeladores de recursos hídricos enfrentan el desafío de
trabajar con diferentes tipos de incertidumbre debido a la
falta de un completo conocimiento de los sistemas naturales,
procesos de modelación, aproximaciones numéricas
(ecuaciones, parámetros, estructuras, soluciones), y datos
de terreno tomados para desarrollar y evaluar modelos.
La propagación de la incertidumbre paramétrica en los
resultados de las simulaciones es un tópico relevante en la
hidrología ambiental. Los análisis de incertidumbre mejoran
la evaluación en el modelamiento hidrológico. Existe una
necesidad en la hidrología moderna de desarrollar y evaluar
métodosdeanálisis deincertidumbrequeapoyen la evaluación
de los modelos hidrológicos. En esta investigación se evaluó
la propagación de la incertidumbre de los parámetros de un
modelo en los resultados del flujo simulado. Un programa de
simulación hidrológico HSPF patrocinado por la Agencia
Ambiental de los EE.UU., fue evaluado utilizando datos
hidroambientales de la cuenca de la quebrada Luxapallila
localizada en los estados de Misisipi y Alabama, EE.UU.
Los límites de incertidumbre de las salidas del modelo fueron
calculados utilizando los métodos de simulación Monte Carlo
y el método probabilístico de estimación puntual de Harr. El
análisis de la propagación de la incertidumbre paramétrica
en simulaciones de caudales con HSPF utilizando 12
parámetros fue realizada con 5000 muestras aleatorias de
Monte Carlo y 24 puntos seleccionados de Harr para cada
parámetro evaluado. La comparación mostró que el método
de Harr podría ser un indicador inicial apropiado de la
propagacióndela incertidumbreparamétrica en simulaciones
de caudales, particularmente en modelos hidrológicos con
varios parámetros.

sippi State University, 501 Hardy Road, 235 Walker Engineering
Los modeladores de recursos hídricos enfrentan el desafío de
trabajar con diferentes tipos de incertidumbre debido a la
falta de un completo conocimiento de los sistemas naturales,
procesos de modelación, aproximaciones numéricas
(ecuaciones, parámetros, estructuras, soluciones), y datos
de terreno tomados para desarrollar y evaluar modelos.
La propagación de la incertidumbre paramétrica en los
resultados de las simulaciones es un tópico relevante en la
hidrología ambiental. Los análisis de incertidumbre mejoran
la evaluación en el modelamiento hidrológico. Existe una
necesidad en la hidrología moderna de desarrollar y evaluar
métodosdeanálisis deincertidumbrequeapoyen la evaluación
de los modelos hidrológicos. En esta investigación se evaluó
la propagación de la incertidumbre de los parámetros de un
modelo en los resultados del flujo simulado. Un programa de
simulación hidrológico HSPF patrocinado por la Agencia
Ambiental de los EE.UU., fue evaluado utilizando datos
hidroambientales de la cuenca de la quebrada Luxapallila
localizada en los estados de Misisipi y Alabama, EE.UU.
Los límites de incertidumbre de las salidas del modelo fueron
calculados utilizando los métodos de simulación Monte Carlo
y el método probabilístico de estimación puntual de Harr. El
análisis de la propagación de la incertidumbre paramétrica
en simulaciones de caudales con HSPF utilizando 12
parámetros fue realizada con 5000 muestras aleatorias de
Monte Carlo y 24 puntos seleccionados de Harr para cada
parámetro evaluado. La comparación mostró que el método
de Harr podría ser un indicador inicial apropiado de la
propagacióndela incertidumbreparamétrica en simulaciones
de caudales, particularmente en modelos hidrológicos con
varios parámetros.

sippi State University, 501 Hardy Road, 235 Walker Engineering
Los modeladores de recursos hídricos enfrentan el desafío de
trabajar con diferentes tipos de incertidumbre debido a la
falta de un completo conocimiento de los sistemas naturales,
procesos de modelación, aproximaciones numéricas
(ecuaciones, parámetros, estructuras, soluciones), y datos
de terreno tomados para desarrollar y evaluar modelos.
La propagación de la incertidumbre paramétrica en los
resultados de las simulaciones es un tópico relevante en la
hidrología ambiental. Los análisis de incertidumbre mejoran
la evaluación en el modelamiento hidrológico. Existe una
necesidad en la hidrología moderna de desarrollar y evaluar
métodosdeanálisis deincertidumbrequeapoyen la evaluación
de los modelos hidrológicos. En esta investigación se evaluó
la propagación de la incertidumbre de los parámetros de un
modelo en los resultados del flujo simulado. Un programa de
simulación hidrológico HSPF patrocinado por la Agencia
Ambiental de los EE.UU., fue evaluado utilizando datos
hidroambientales de la cuenca de la quebrada Luxapallila
localizada en los estados de Misisipi y Alabama, EE.UU.
Los límites de incertidumbre de las salidas del modelo fueron
calculados utilizando los métodos de simulación Monte Carlo
y el método probabilístico de estimación puntual de Harr. El
análisis de la propagación de la incertidumbre paramétrica
en simulaciones de caudales con HSPF utilizando 12
parámetros fue realizada con 5000 muestras aleatorias de
Monte Carlo y 24 puntos seleccionados de Harr para cada
parámetro evaluado. La comparación mostró que el método
de Harr podría ser un indicador inicial apropiado de la
propagacióndela incertidumbreparamétrica en simulaciones
de caudales, particularmente en modelos hidrológicos con
varios parámetros.

Palabras clave: HSPF, incertidumbreparamétrica, simulación Monte Carlo, método de estimación puntal de Harr, límites de incertidumbre de simulaciones de caudales 


\section{Introduction}

Hydrologic models are represented by a series of input data (e.g. precipitation and evaporation), parameters (e.g. soil, land use, and channel properties), and structure (e.g. black-box, conceptual, physically based, grid, and lumped models). Every component of hydrologic models depicts uncertainty due to the lack of knowledge about real systems. Uncertainty in input data is due to natural variability, measurement inaccuracy, and errors in handling and processing data (Melching, 1995). Model parameters and structure show uncertainty due to model assumptions and approximations, scale effects, and variability of inputs and parameters in time and space (Gupta et al., 2005; Tung, 1996). The uncertainty of input data on model results has been studied separately from model parameter uncertainty (Souid, 1999). Georgakakos et al. (2004) pointed out that few studies have investigated model structure uncertainty. Butts et al. (2004) declared that uncertainty evaluation is compensated among components (input data, model parameters, and model structure) because they are strongly interlinked.

The identification, quantification, and reporting of the different sources of errors in a modeling process constitute an uncertainty analysis (McIntyre et al., 2002; Refsgaard and Henriksen 2004; Refsgaard et al., 2007). Uncertainty analysis has received considerable attention during the last two decades by the water resources community. In hydrological modeling, important progress has been observed in the identification and understanding of the different sources of uncertainty, as well as in the incorporation of strategies for their quantification (e.g. Butts et al., 2004; Georgakakos et al., 2004; Wagener et al., 2003). Uncertainty analysis of computer-based models is a valuable tool to do the following: understand the inability of a model to accurately and precisely depict the real world; enhance the value of information reported; distinguish between bias and precision error; calculate the precision limit of results; identify which components are most and least important; determine where to place more effort/resources to decrease the total uncertainty of the output; re-build a model; understand model limitations and strengths; calculate statistical properties of a model output; determine reliability analysis; and compare and choose between models (Morgan and Henrion, 1990;
Tung, 1996; Tung and Yen, 2005).

Techniques applicable for evaluating error propagation from different sources on hydrology model results can be classified in three groups: first-order methods (Melching, 1992, 1995; Zhang and Yu, 2004); probabilistic point estimate methods (Harr, 1989; Rosenblueth, 1975; Tung and Yen, 2005; Yu et al., 2001;); and Monte Carlo based methods such as Bayesian analysis, Markov Chain Monte Carlo, and the Generalized Likelihood Uncertainty Estimation method (Beven and Binley, 1992; Dilks et al. 1992; Thiemann et al., 2001). Applications and comparisons of these techniques on hydrology models can be found in Rogers et al. (1985), Binley et al. (1991), Melching (1995), and Yu et al. (2001). In general, these studies assumed that the results of the Monte Carlo method were most reliable when estimations from other uncertainty methods were compared. The Monte Carlo simulation is the best known and simplest way of sampling the entire range of likely observations of the system being studied (Morgan and Henrion, 1990). Most of the firstorder and probabilistic point estimate methods are more computationally efficient than the Monte Carlo method. Melching (1995) pointed out that "research is needed to define strengths and weaknesses of applying these methods to computer models of watershed hydrology."

Numerous real world hydrologic models exist, e.g. continuous or event based, distributed or lumped parameters, and empirical or physical equations (Singh, 1995; Singh and Woolhiser, 2002; Singh and Frevert, 2002). Currently many continuous hydrologic models are set up in conjunction with Geographical Information Systems GIS. In 1996, the US Environmental Protection Agency EPA released the Better Assessment Science Integrating Point and Nonpoint Sources - BASINS, which links the Hydrological Simulation Program - FORTRAN HSPF (Bicknell et al., 2001) and other watershed and water quality models with a GIS software, MapWindow (USEPA, 2011). Also BASINS incorporates an extensive U.S. data base (i.e. land use, climatological and water quality data) graphical and statistical analysis, and reporting tools. The HSPF software is a continuous, reservoir-type, semidistributed parameter model supported by the USEPA. The HSPF model is one of the most comprehensive, flexible and modular programs of watershed hydrology and water 
quality available (Donigian et al., 1995). HSPF has been applied in different zones around the world since the 1980's (Diaz-Ramirez et al., 2008, 2011; Donigian et al., 1995; Singh and Woolhiser, 2002). Applications of HSPF in watersheds in the southeastern United States can be found in Alarcon et al. (2009), Diaz-Ramirez et al. (2011), and Duan et al. (2008). These studies mainly analyzed hydrological processes on the Luxapallila Creek watershed (Alabama and Mississippi), Saint Louis Bay watershed (Mississippi), Fish River watershed (Alabama), and the Mobile Bay basin (Alabama, Mississippi, Tennessee, and Georgia).

The main goal of this study is to evaluate two uncertainty methods, the Monte Carlo method and Harr's probabilistic point estimate method, in propagating HSPF parameter uncertainty into daily streamflow model results. Physical data from the Luxapallila Creek watershed are used to set up the HSPF model. This watershed is located in Alabama and Mississippi, USA. U.S. Geological Survey USGS streamflow data collected at the watershed outlet from $01 / 01 / 2002$ to $12 / 31 / 2005$ were used to evaluate model results.

\section{The HSPF model}

The Hydrological Simulation Program - FORTRAN HSPF model (Bicknell et al., 2001) computes the movement of water through a complete hydrologic cycle - precipitation (rain/snow), evapotranspiration, runoff, infiltration, and flow through the ground - and the associated transport of constituents with that flow. It represents a watershed as a collection of land segments and channels (reaches). The land segments, either pervious or impervious, are connected to other land segments or to channel reaches, which can function as either streams or reservoirs. Rainfall is computed over the entire watershed and runs off land segments and reaches. Pervious land segments also store water in the plant canopy, on the surface, and in the soil, from which it can percolate into groundwater or flow down slope as interflow. Water in the plant canopy, surface, and surface soil layers can be lost to evapotranspiration. Water in reaches can be lost to evaporation, but not to groundwater. Water can flow from a land segment to a reach or to another land segment. Water in a reach must either be stored there or flow into another reach; it cannot

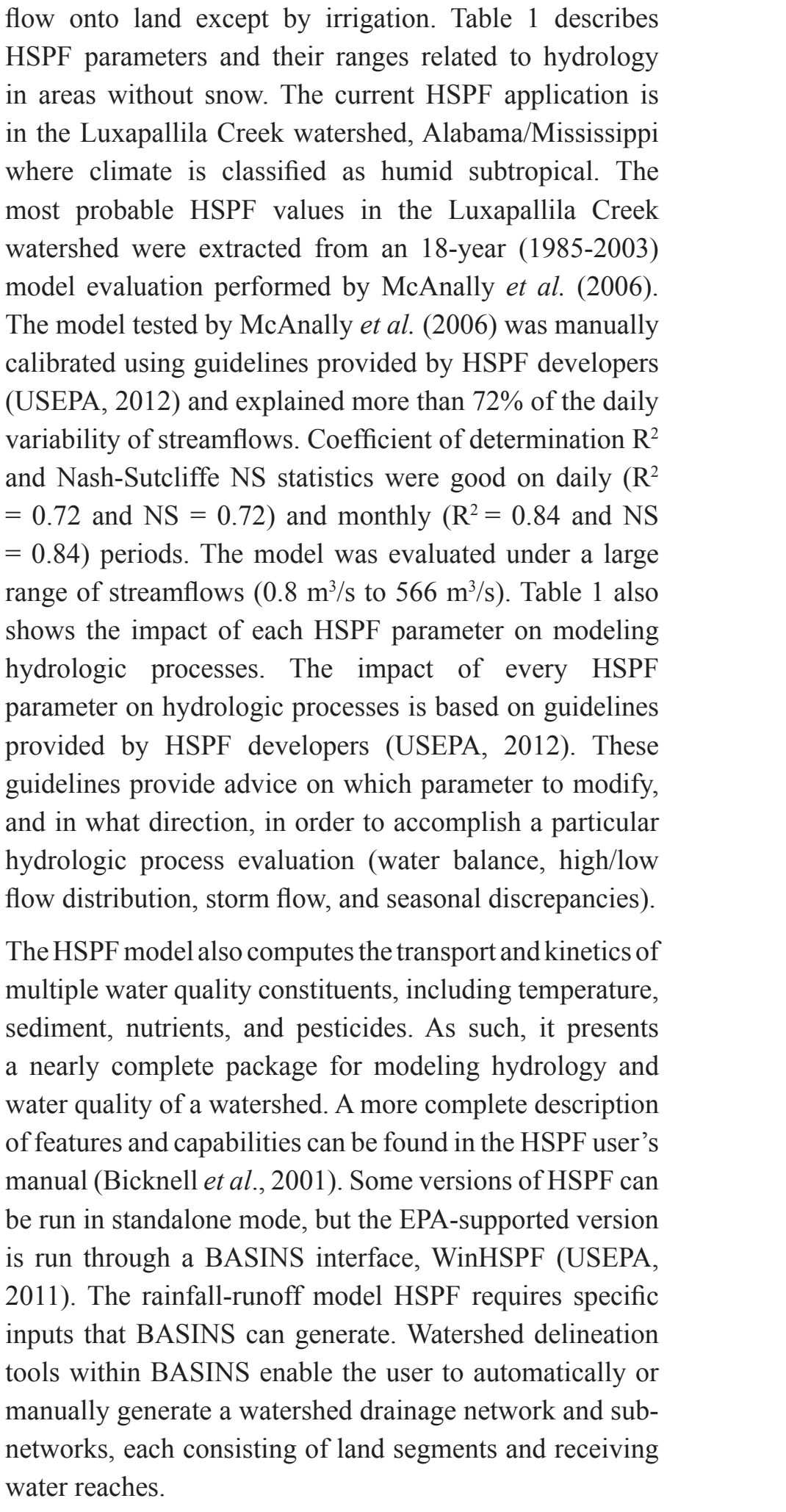

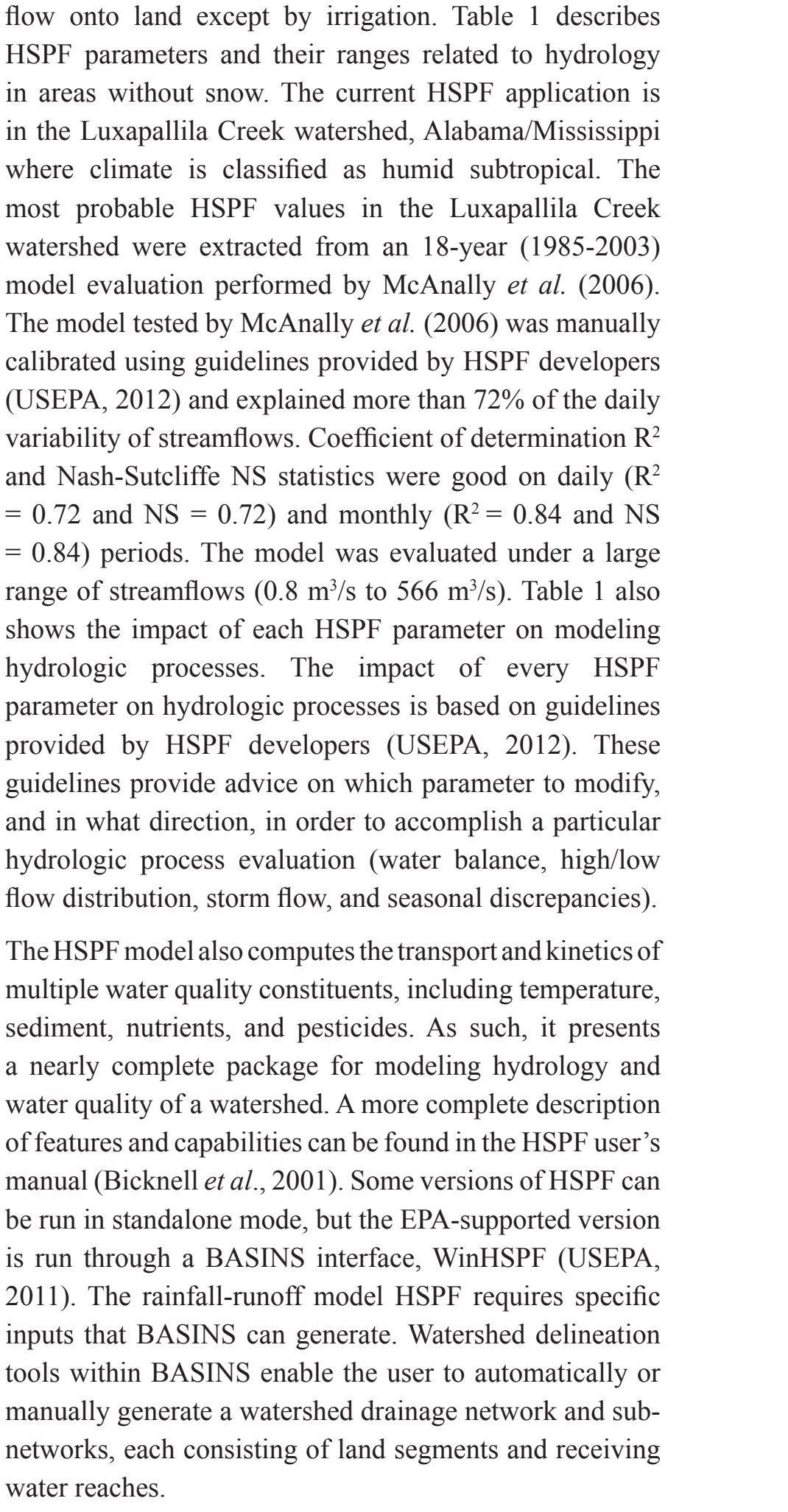

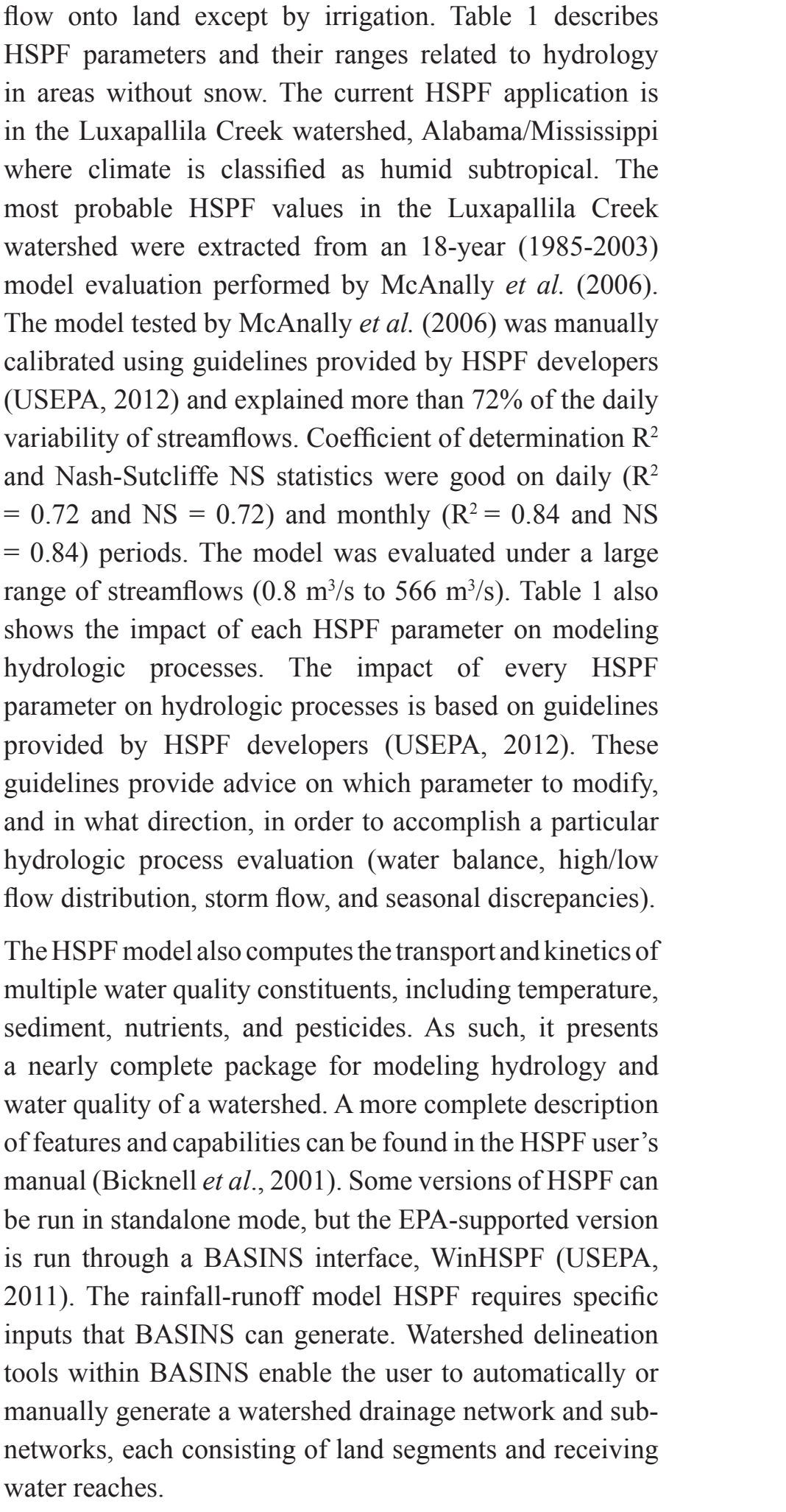

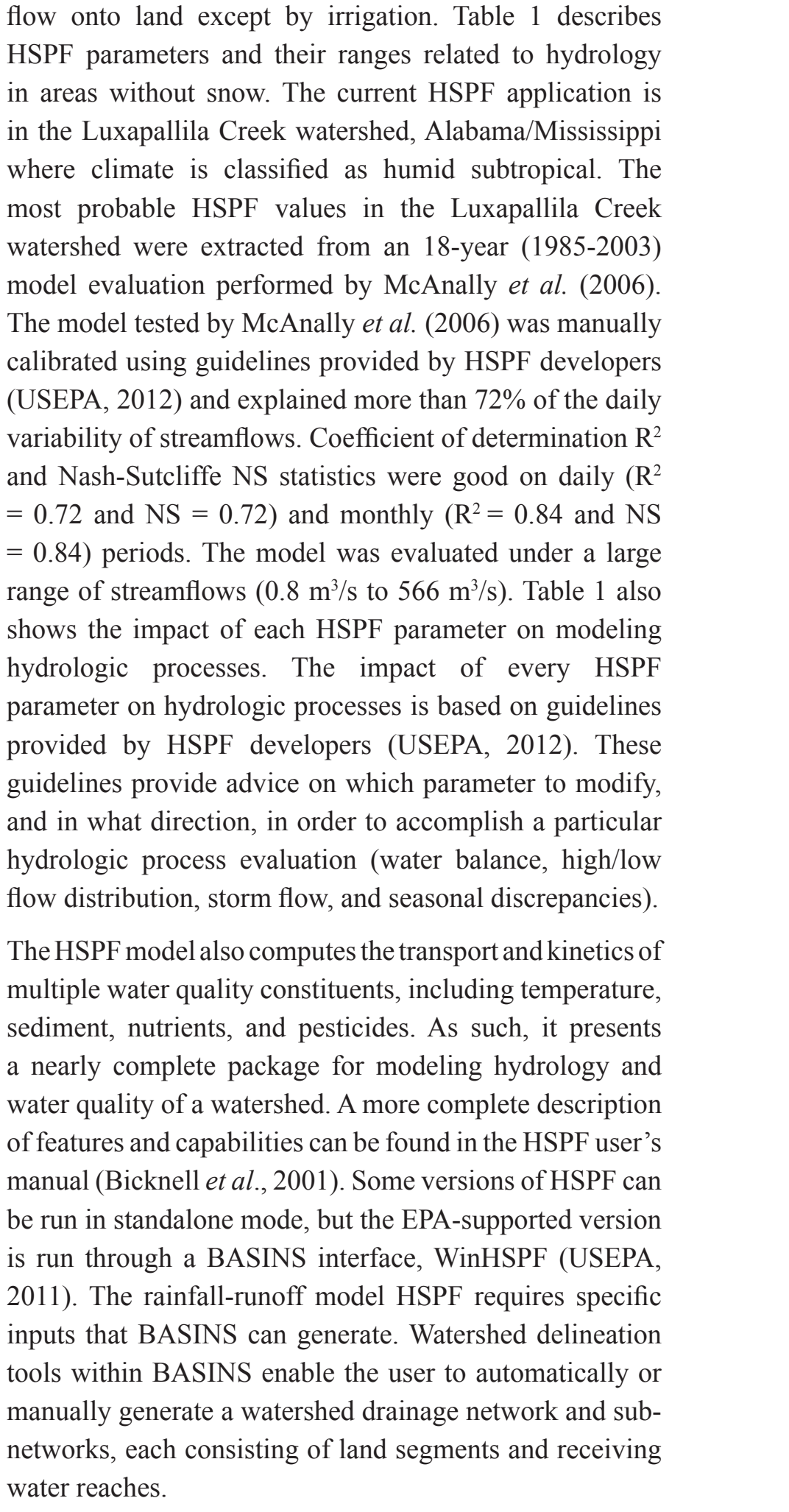

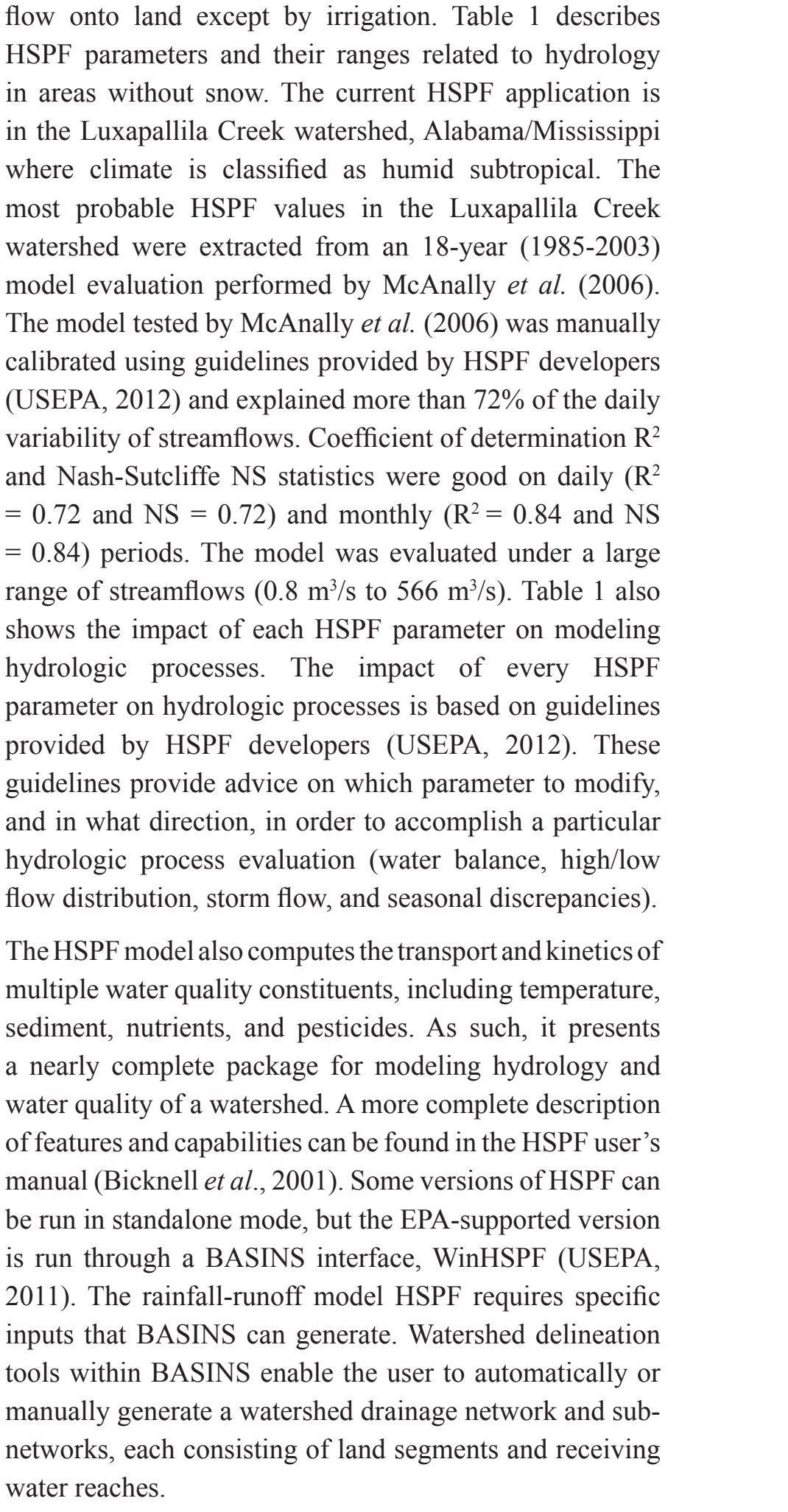

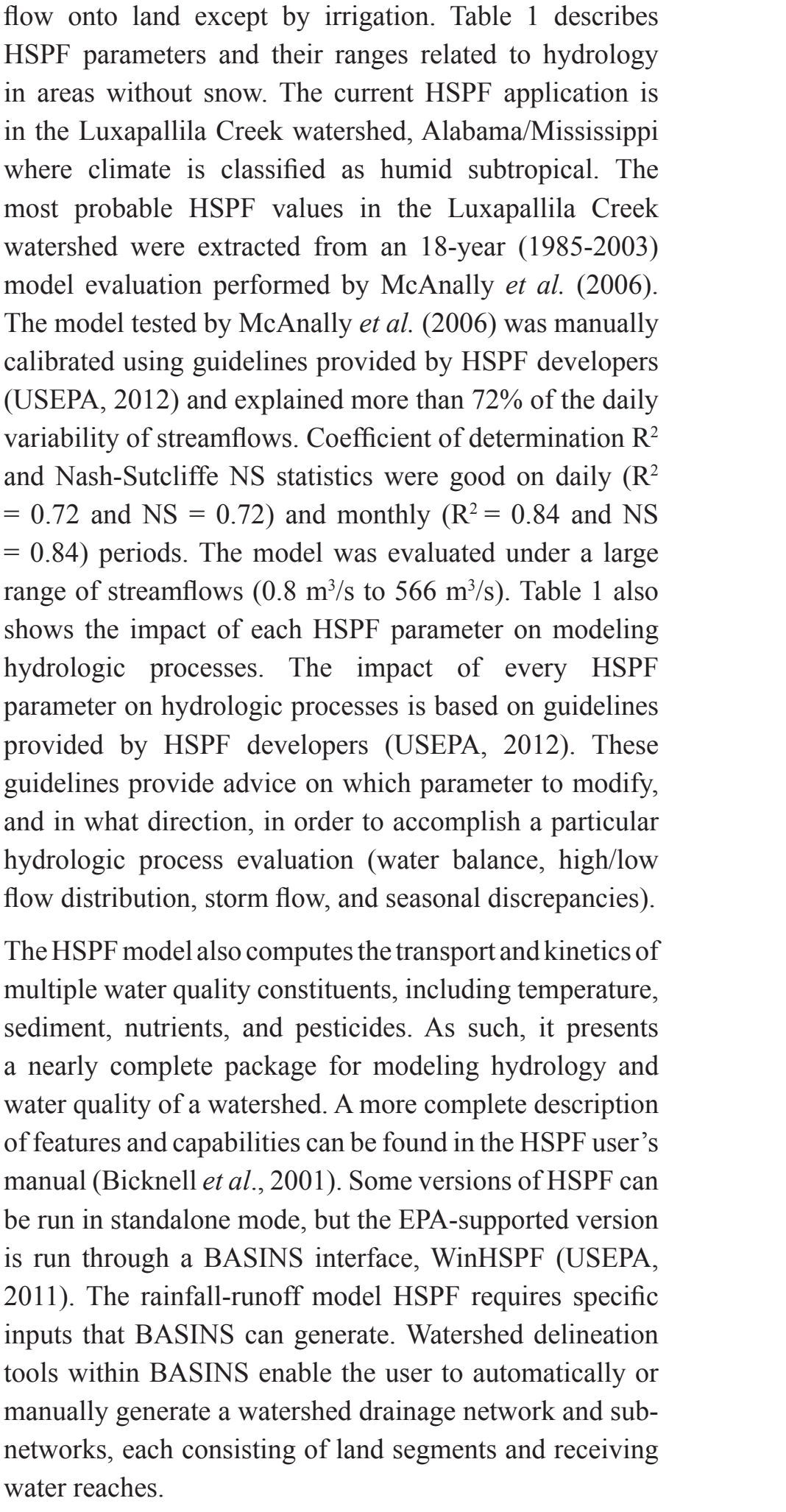

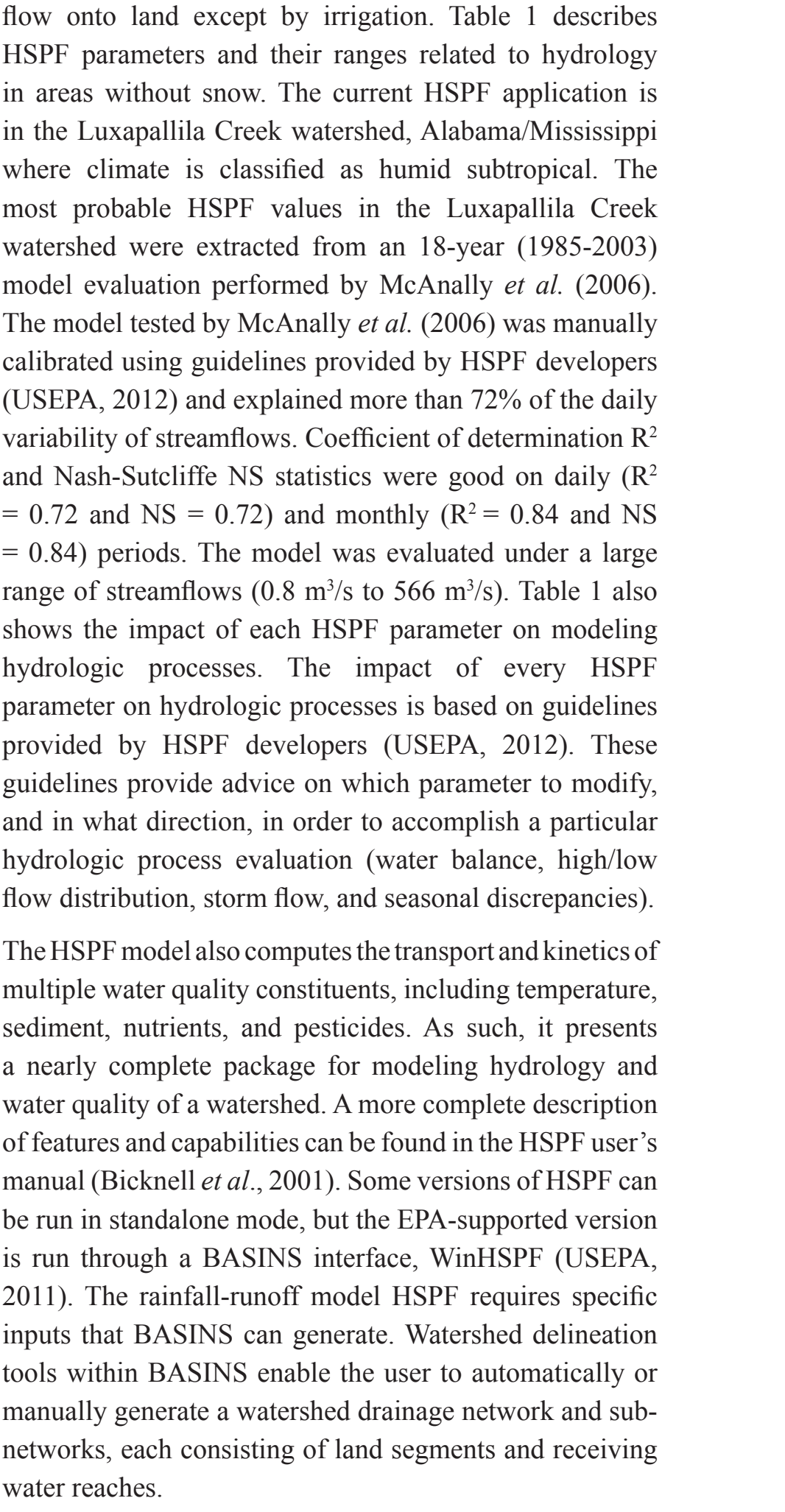

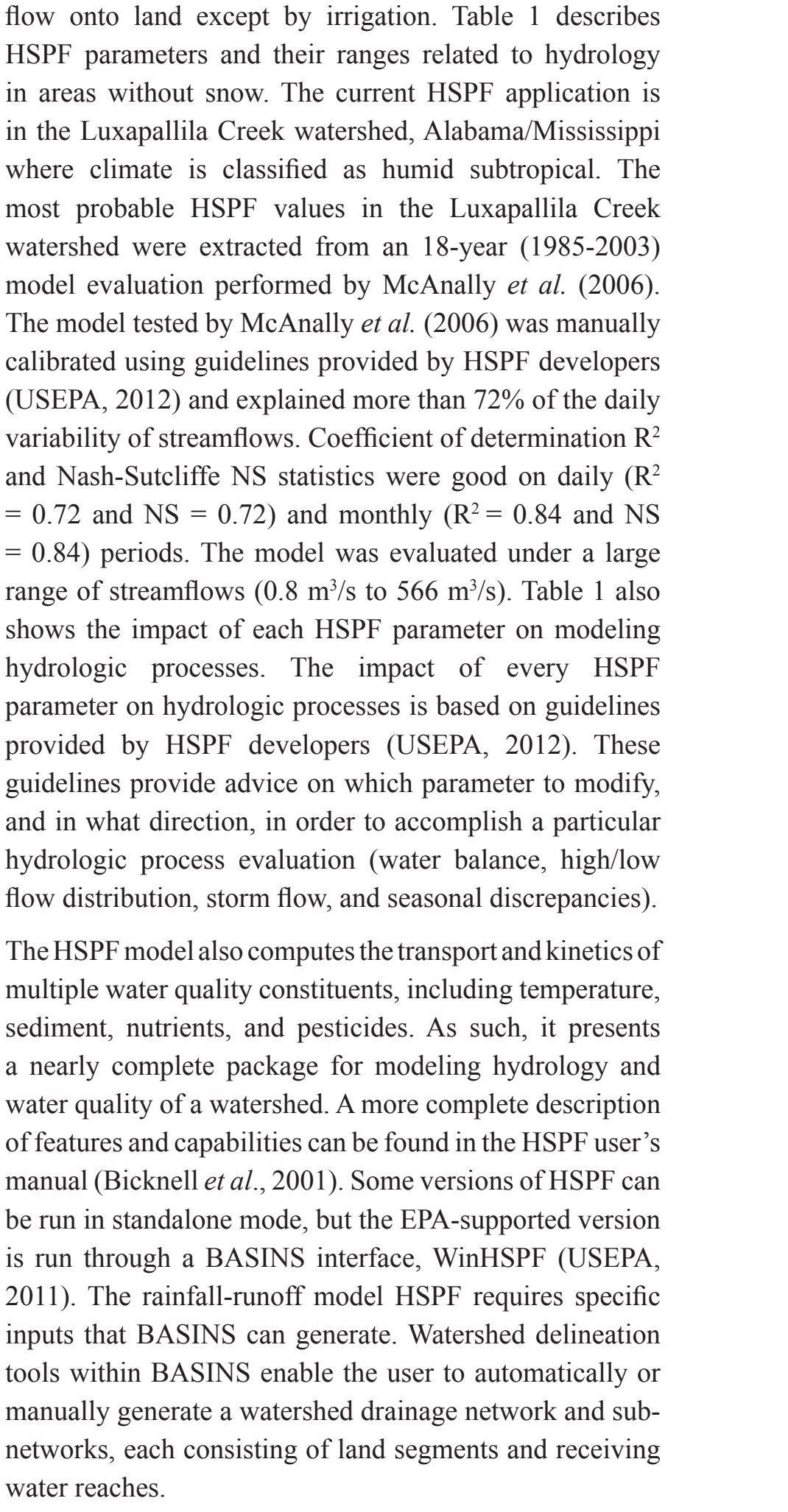

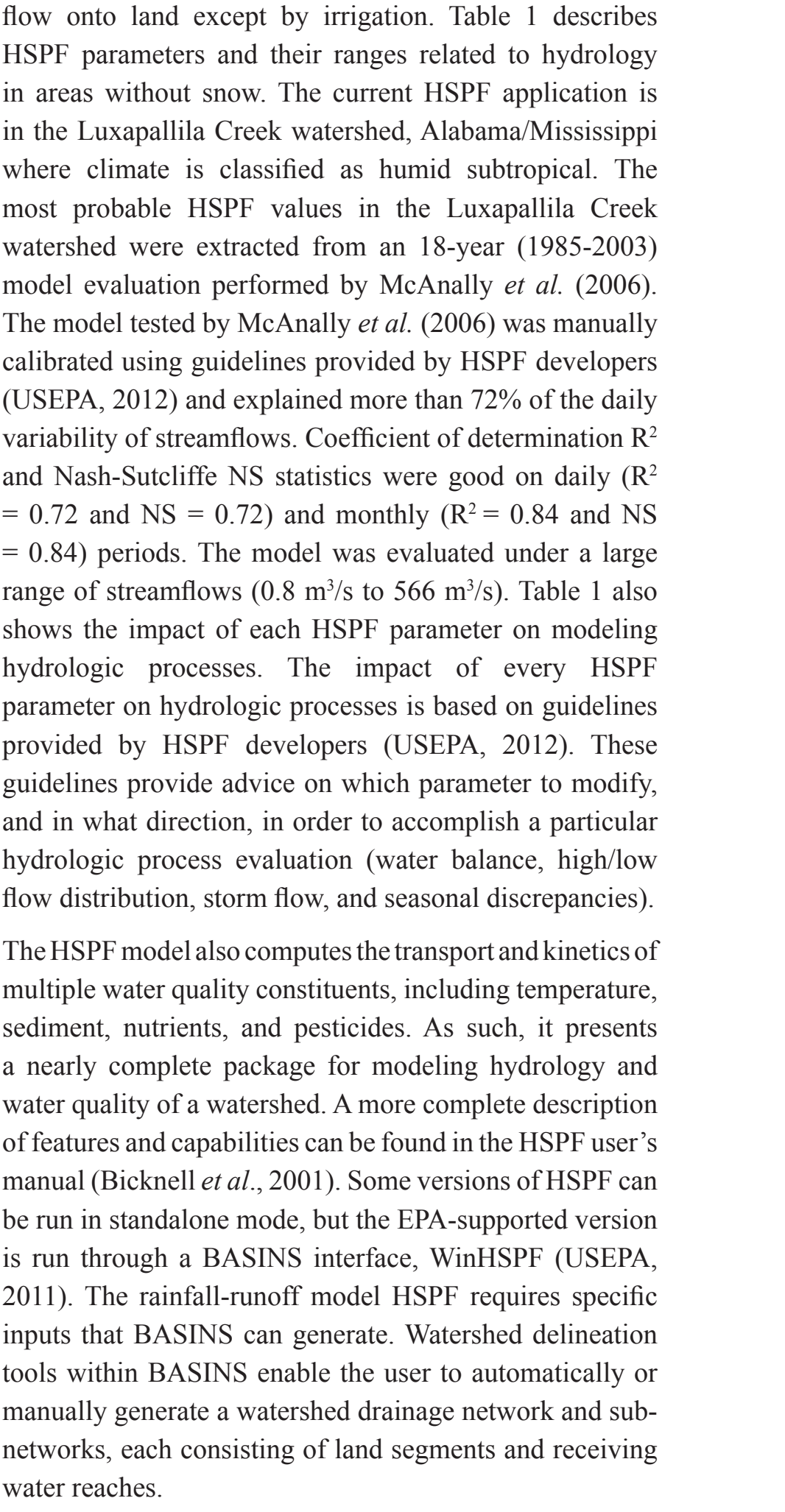

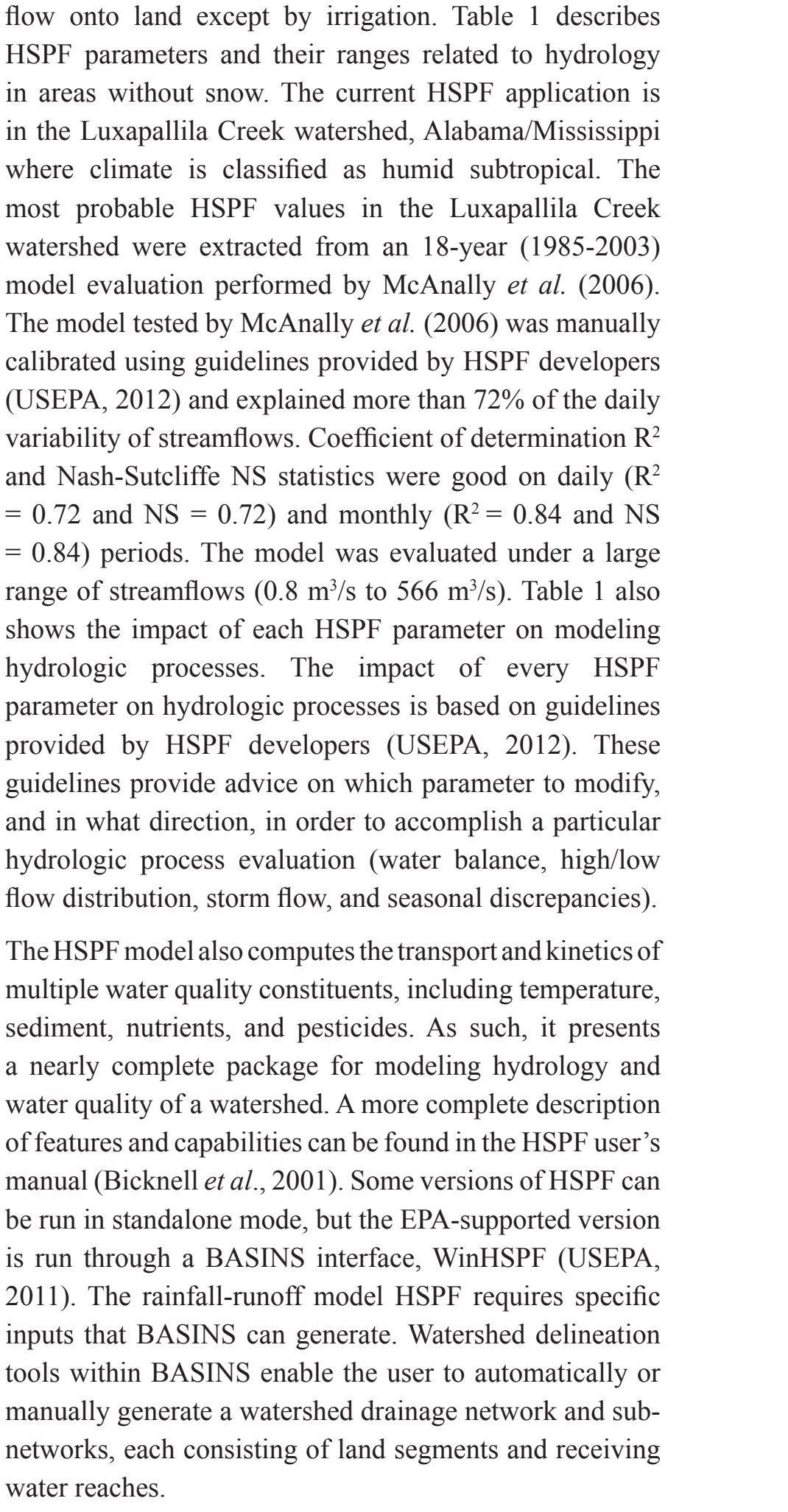

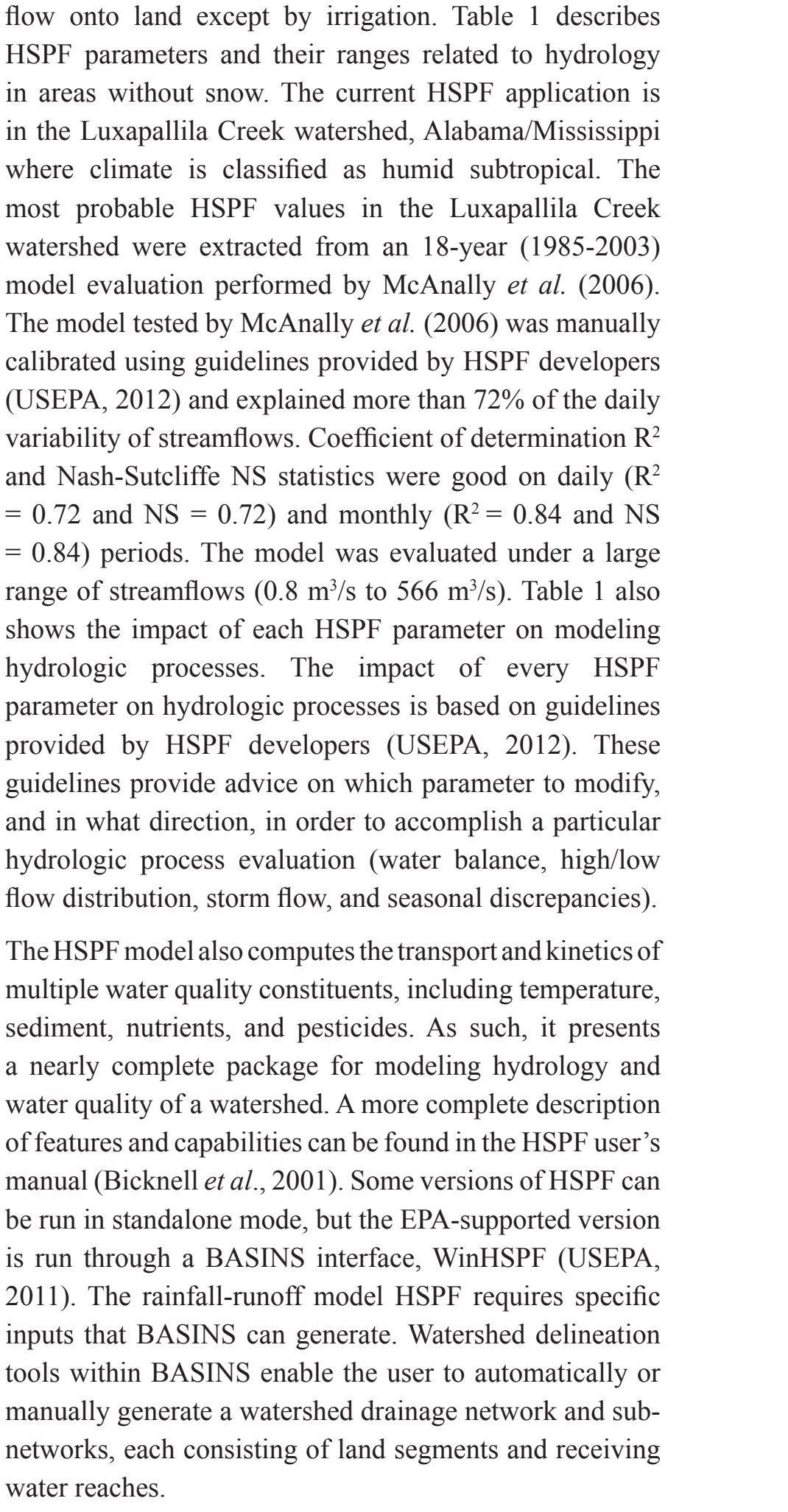

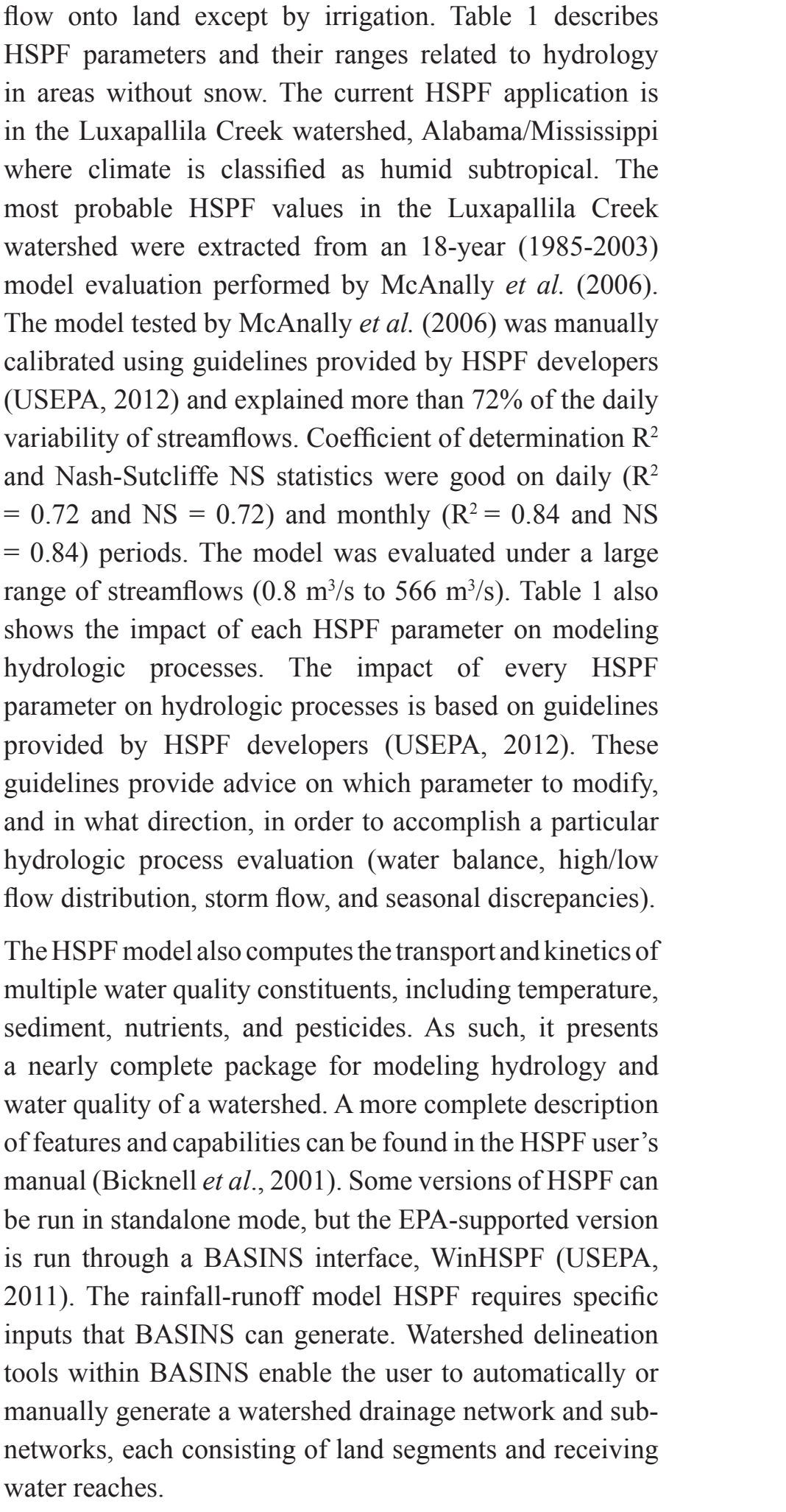

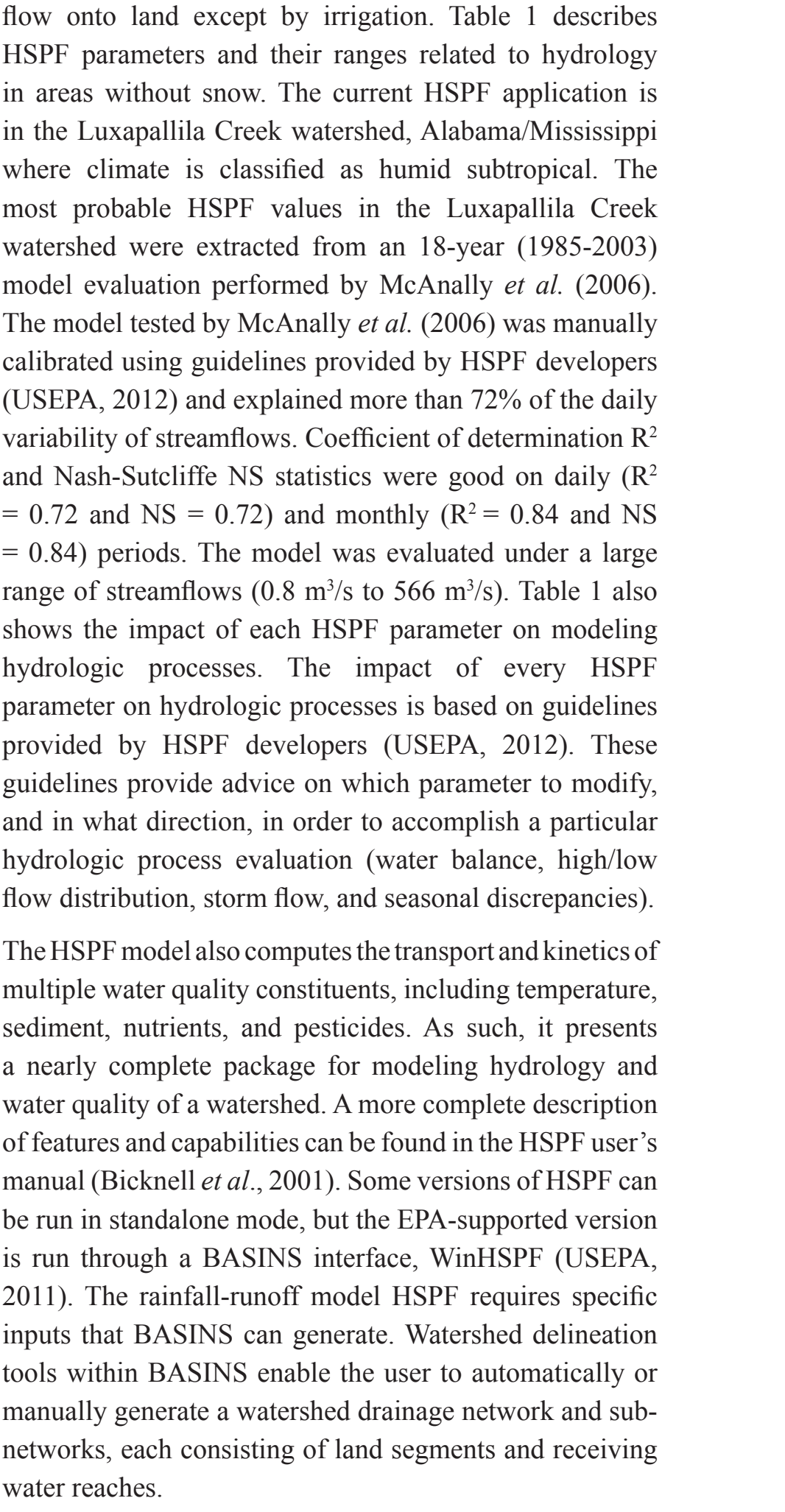

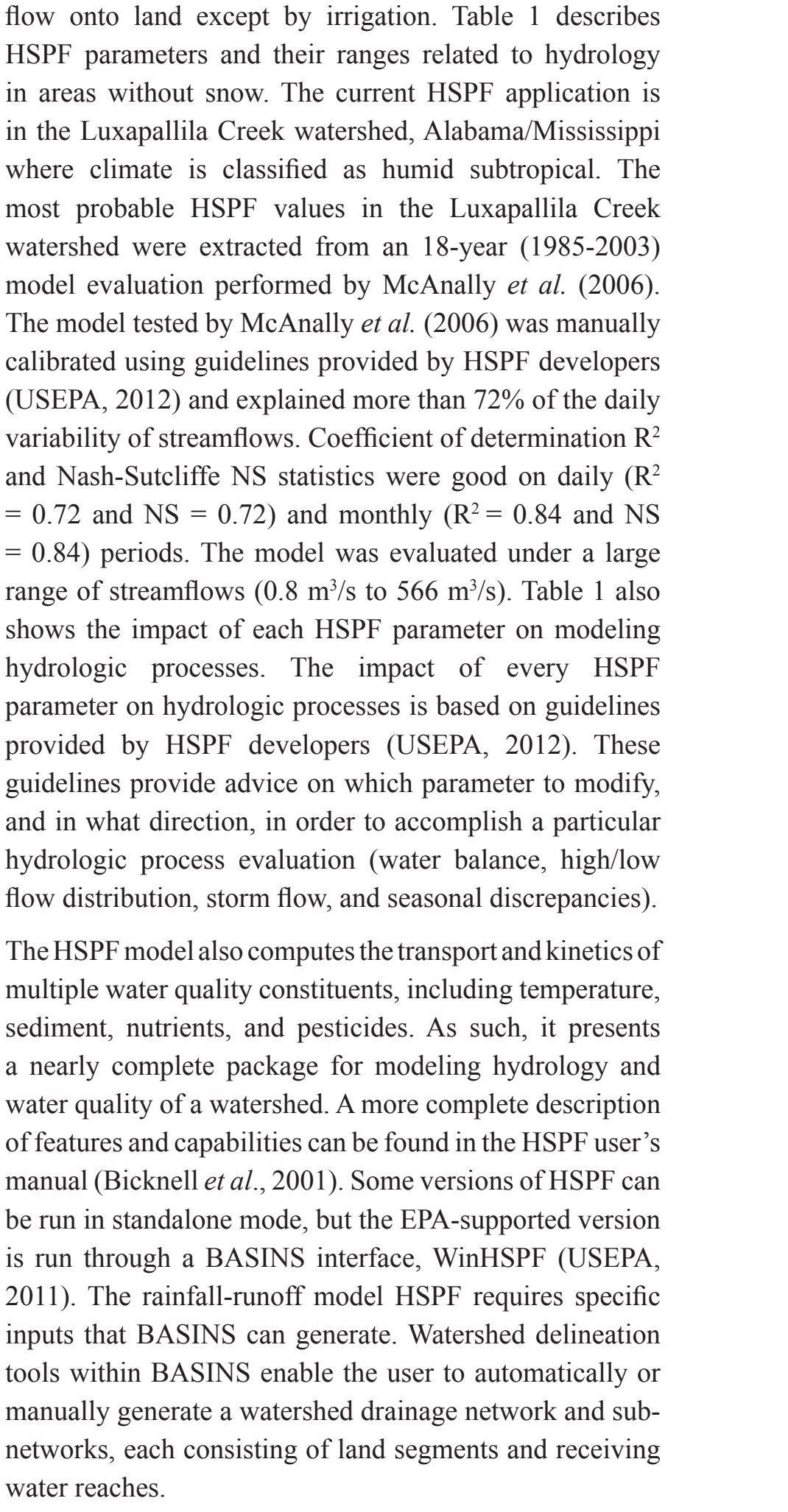

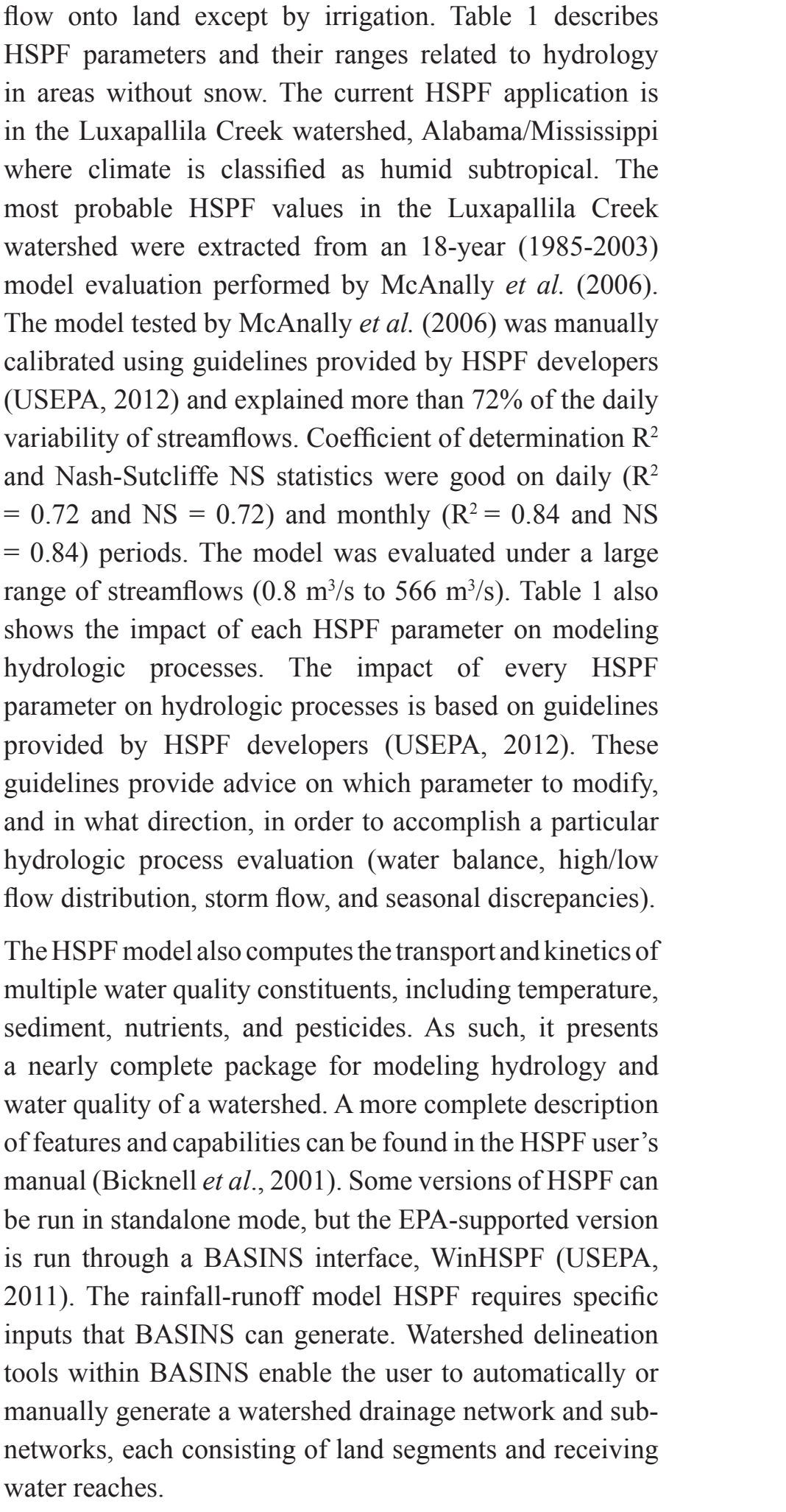

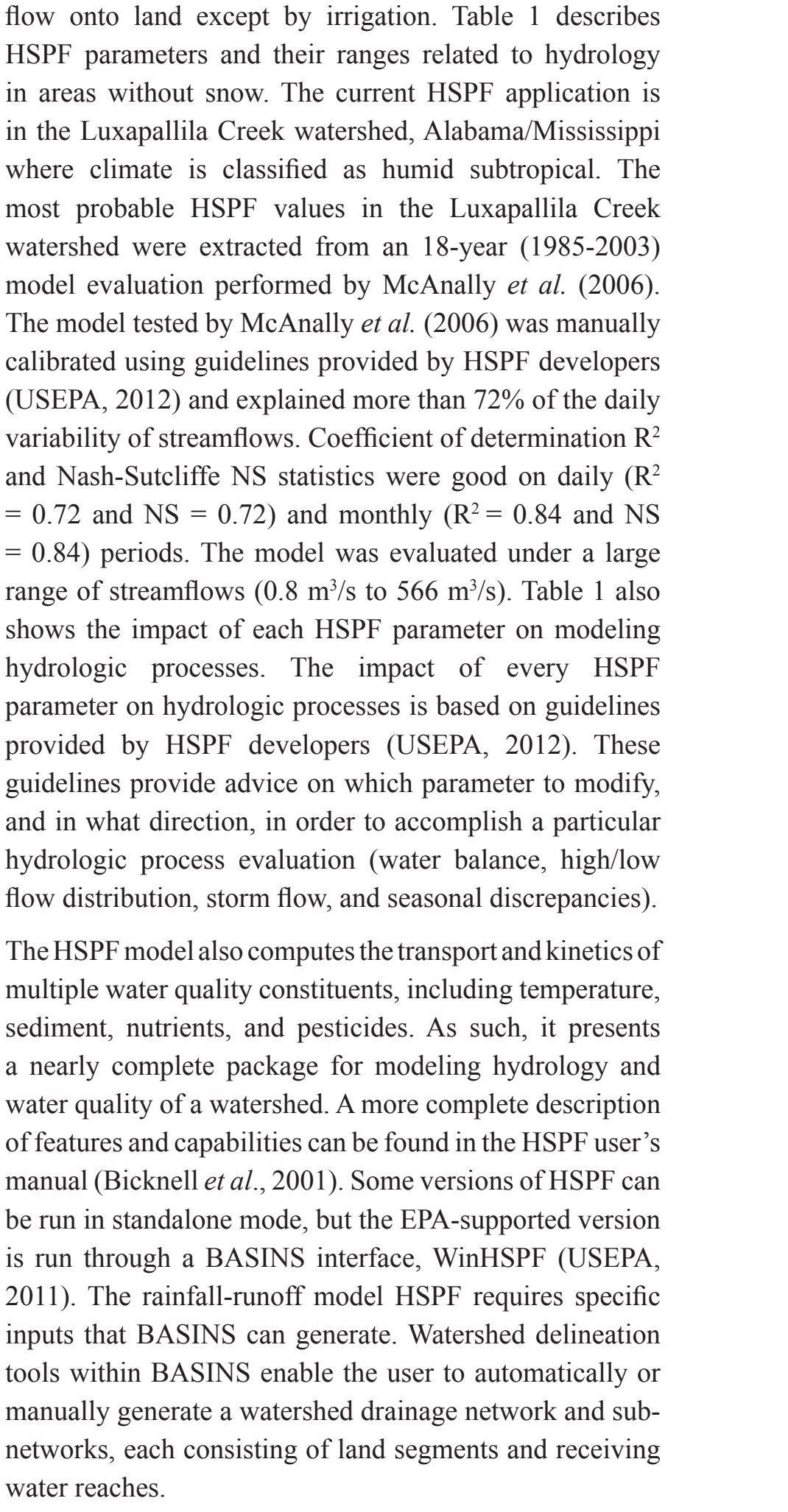

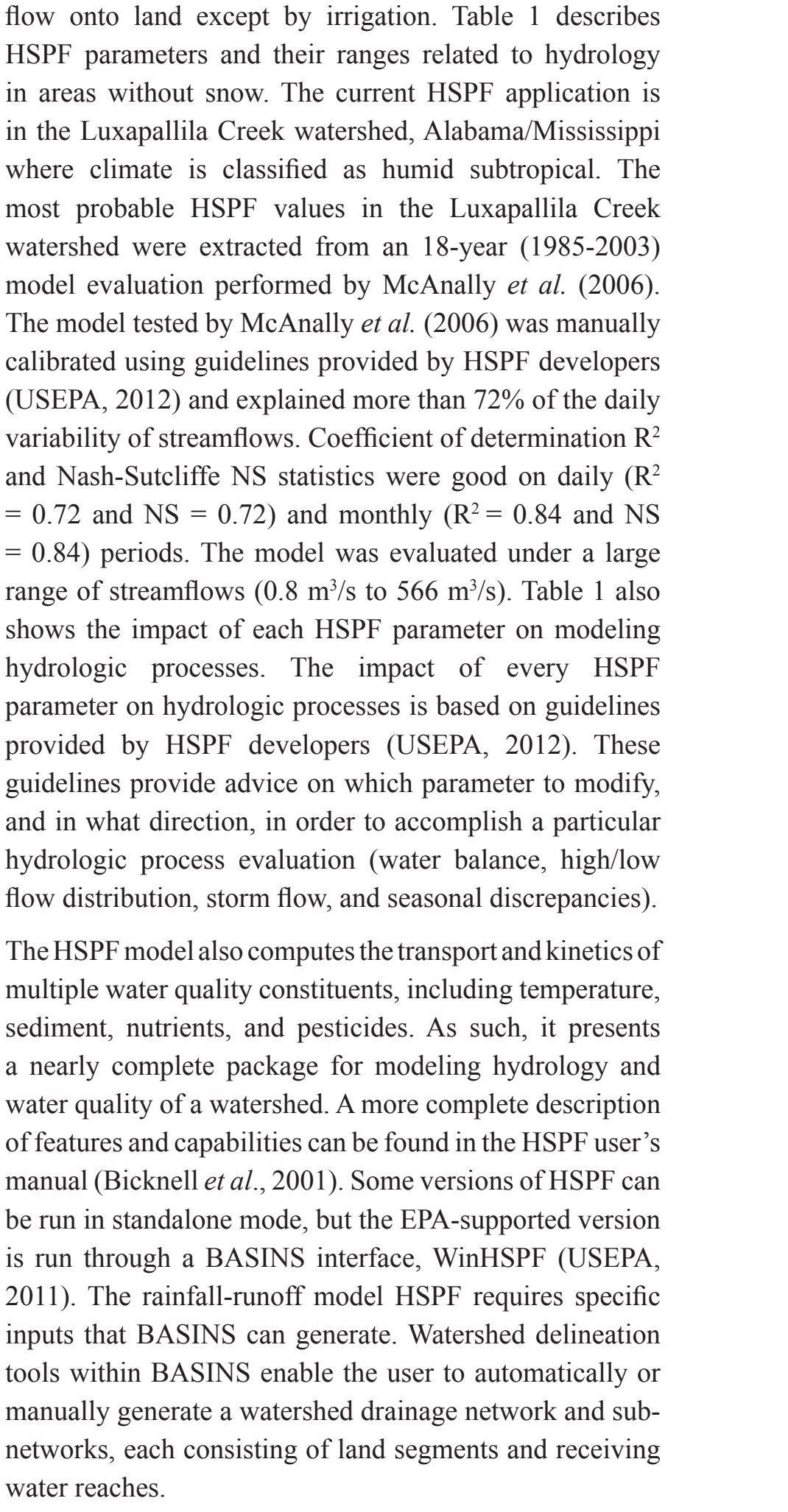

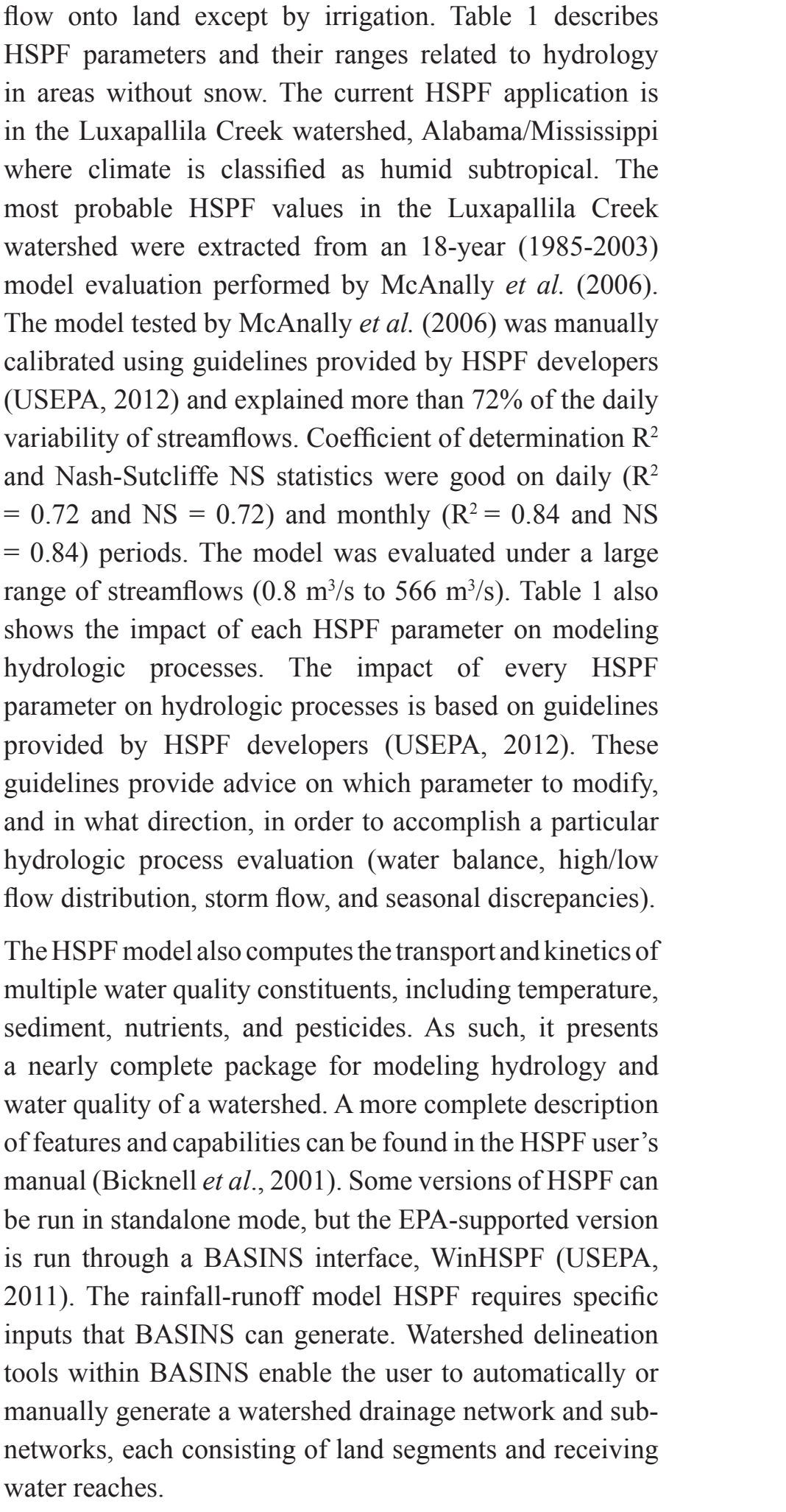

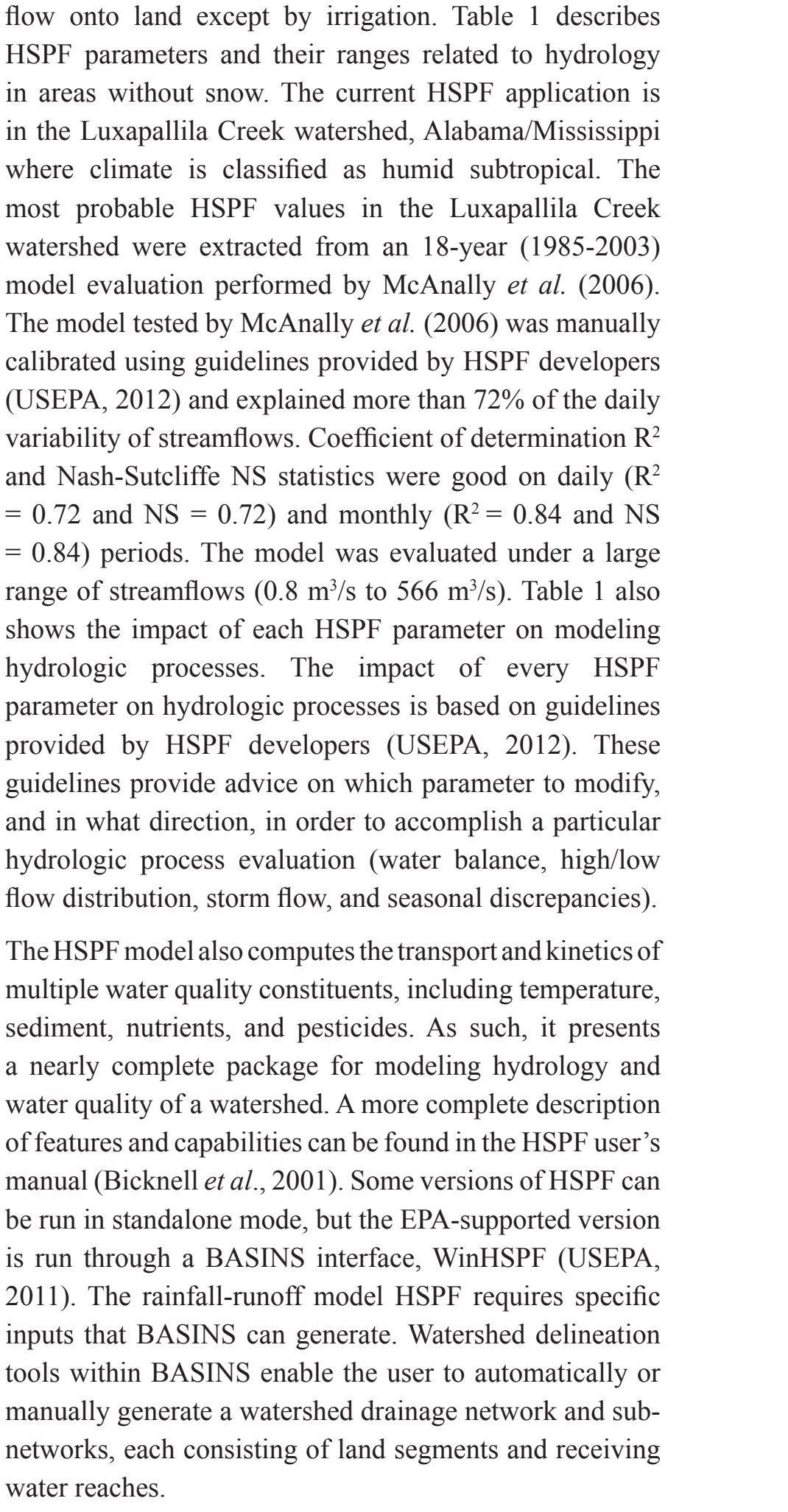

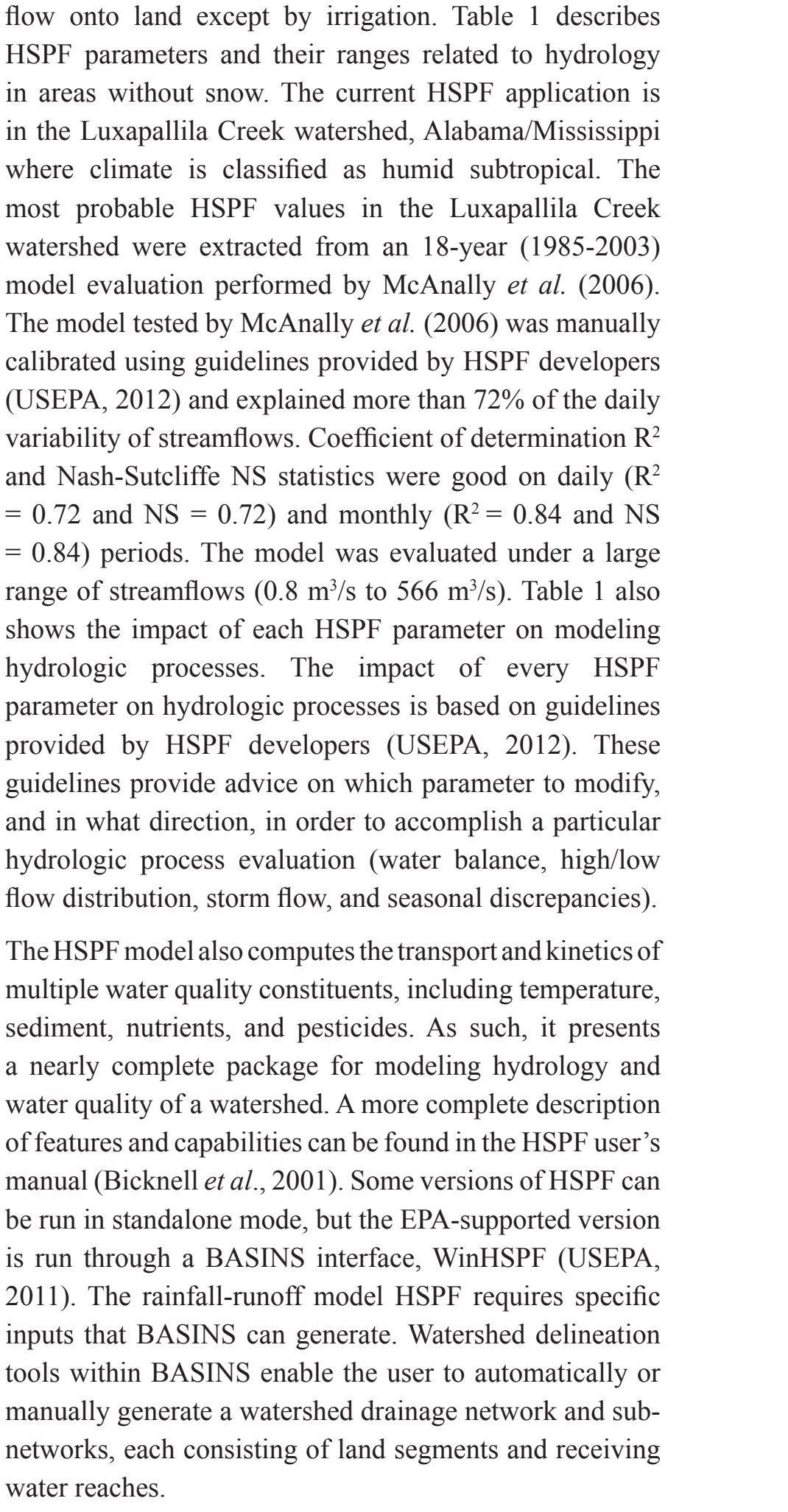

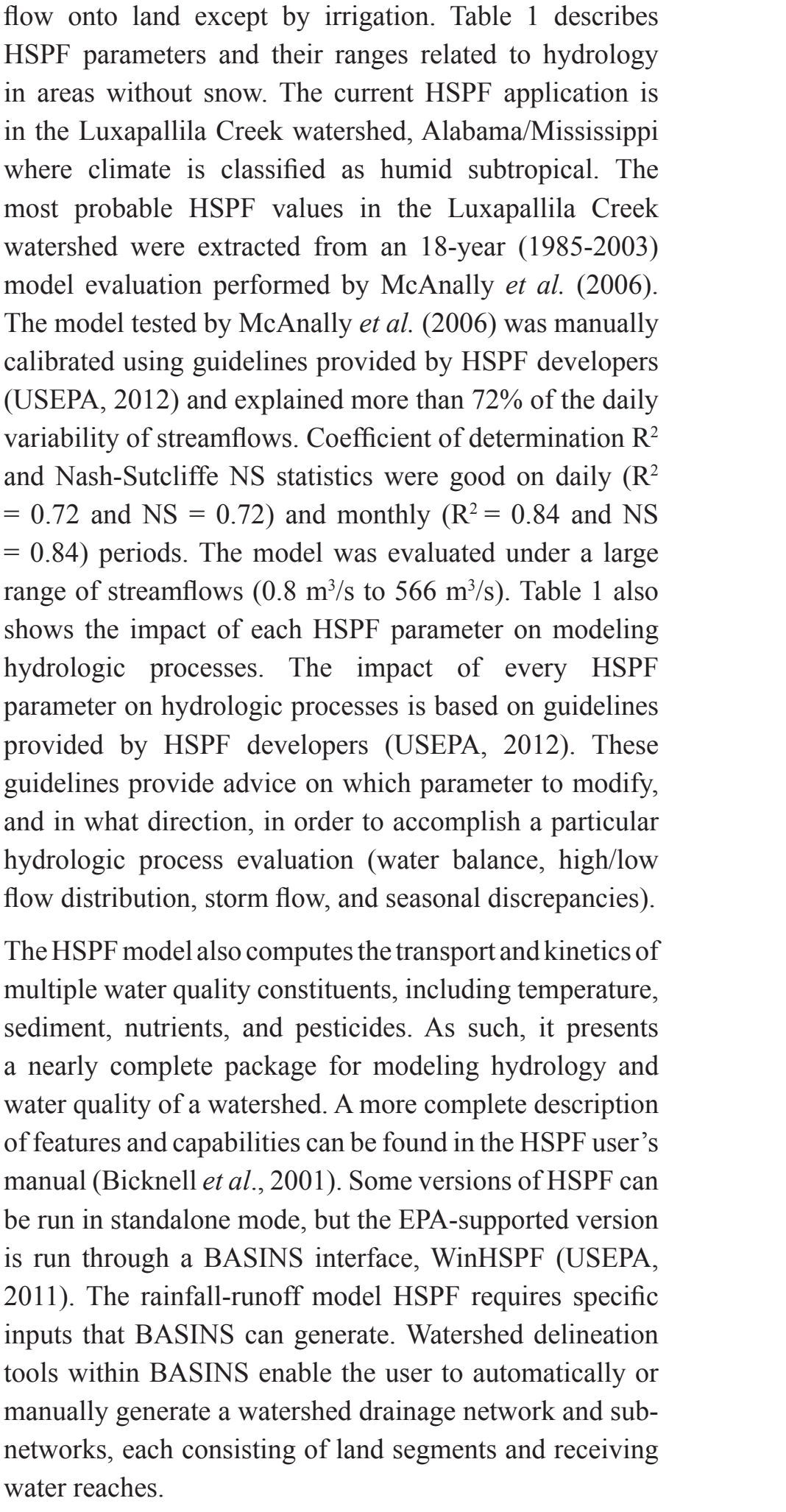

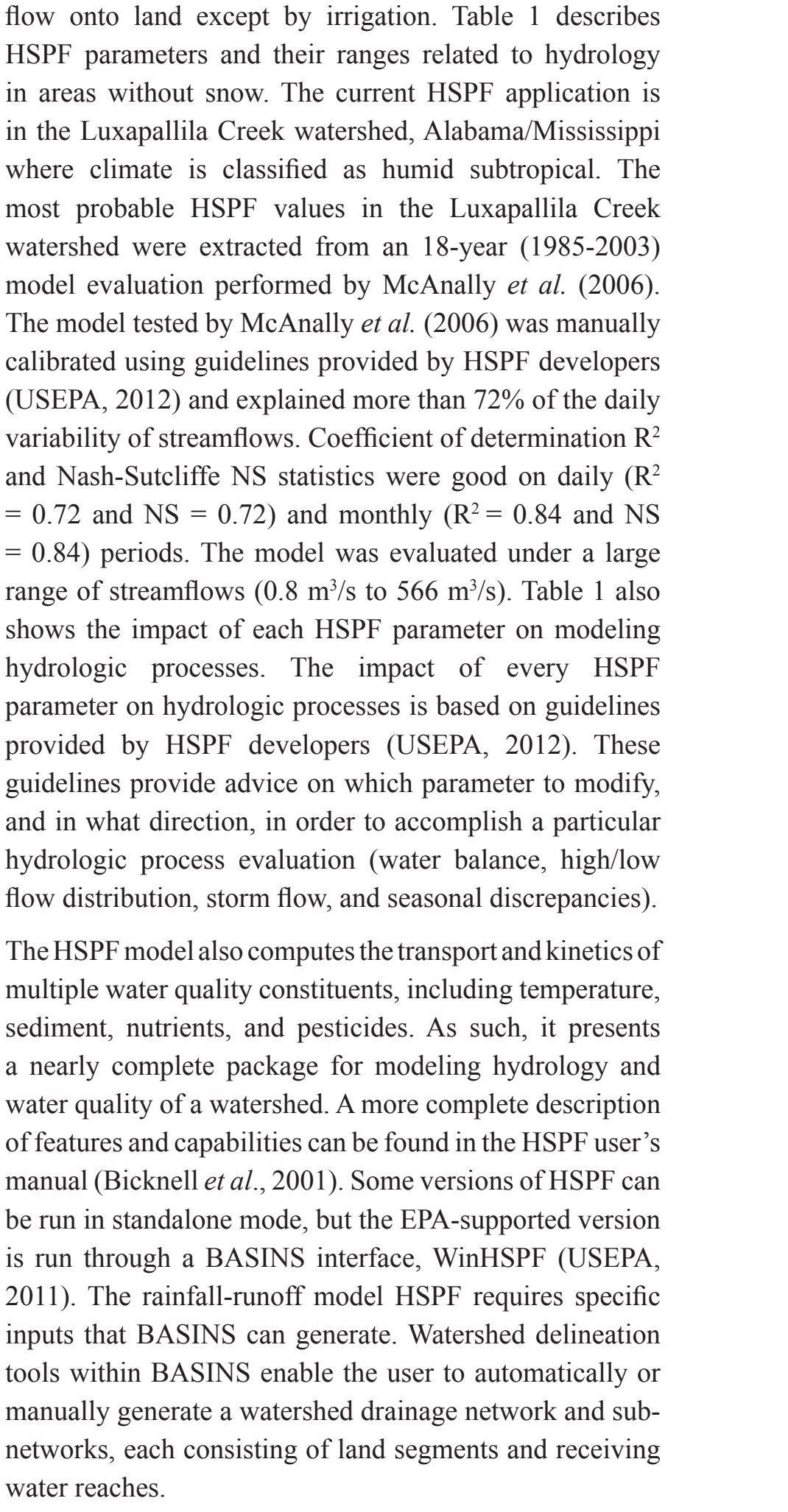

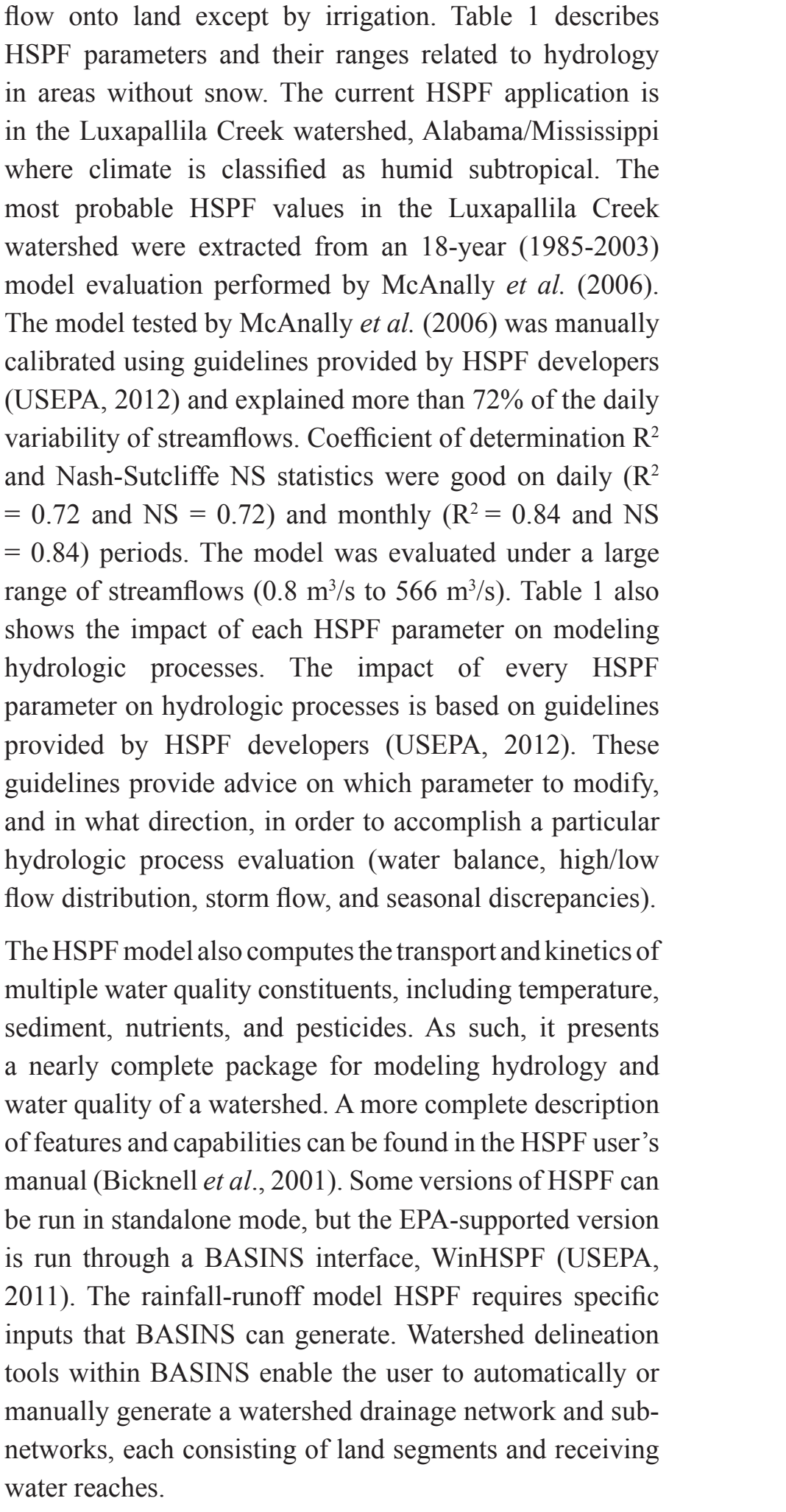

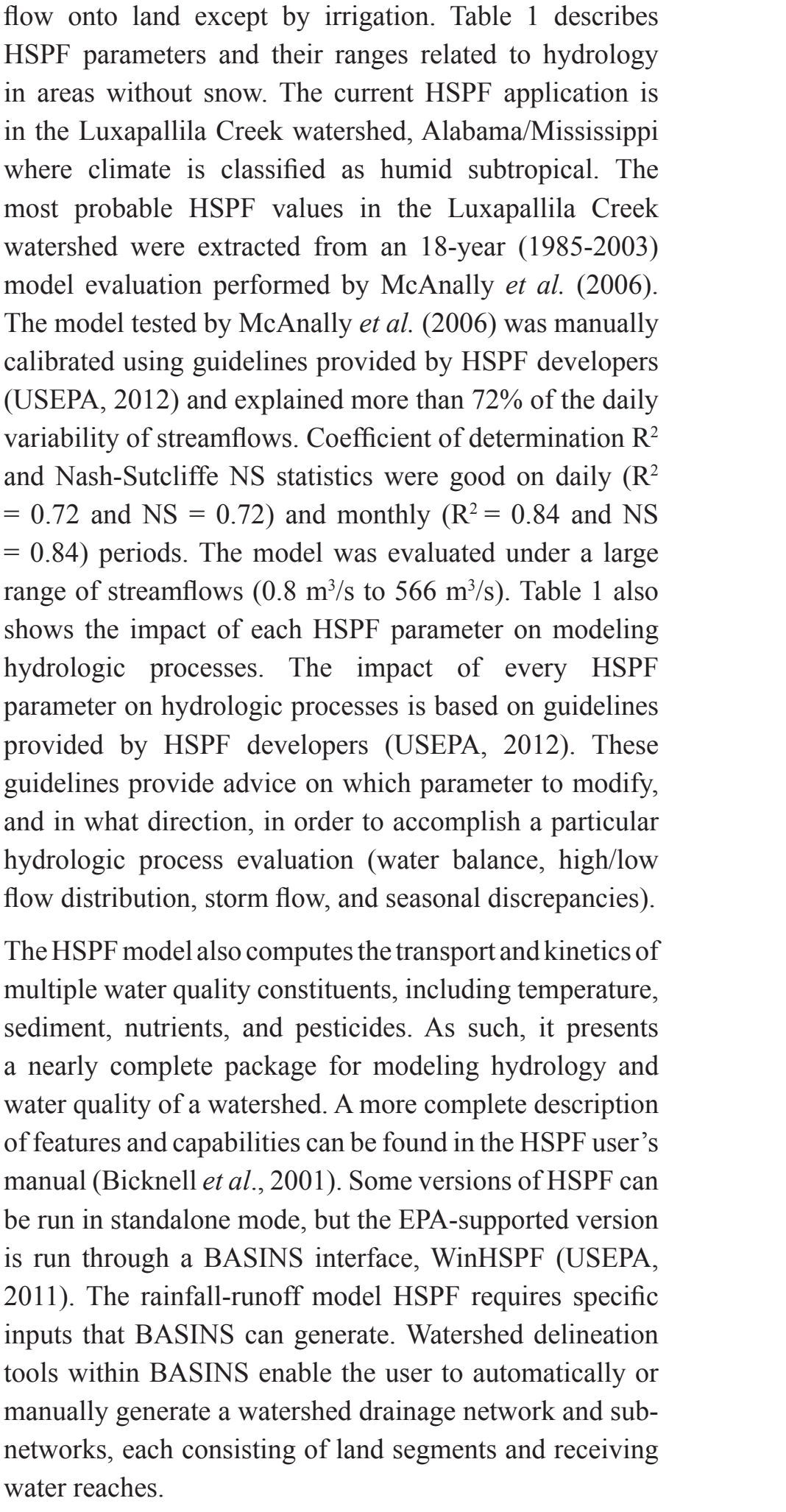

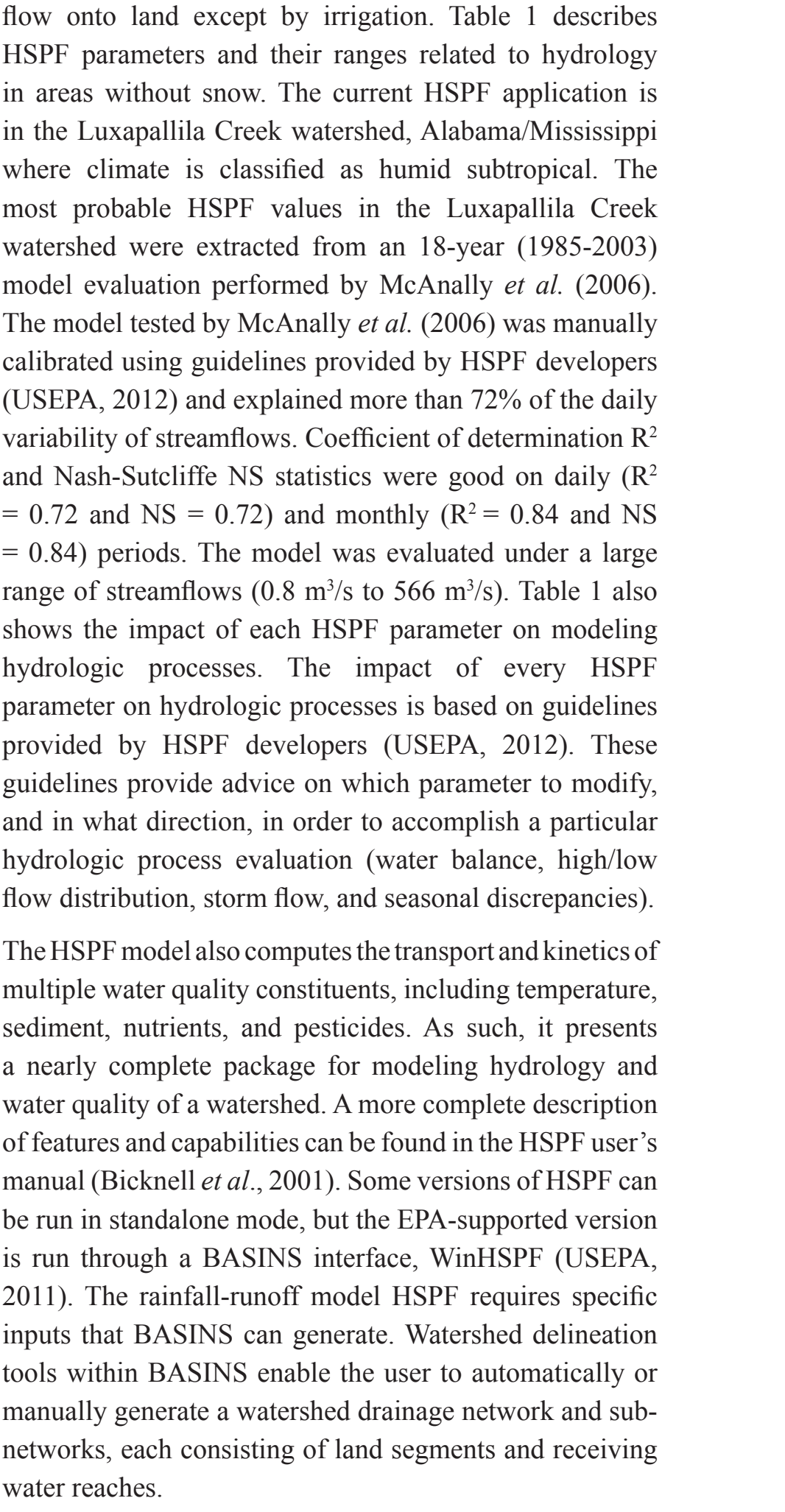

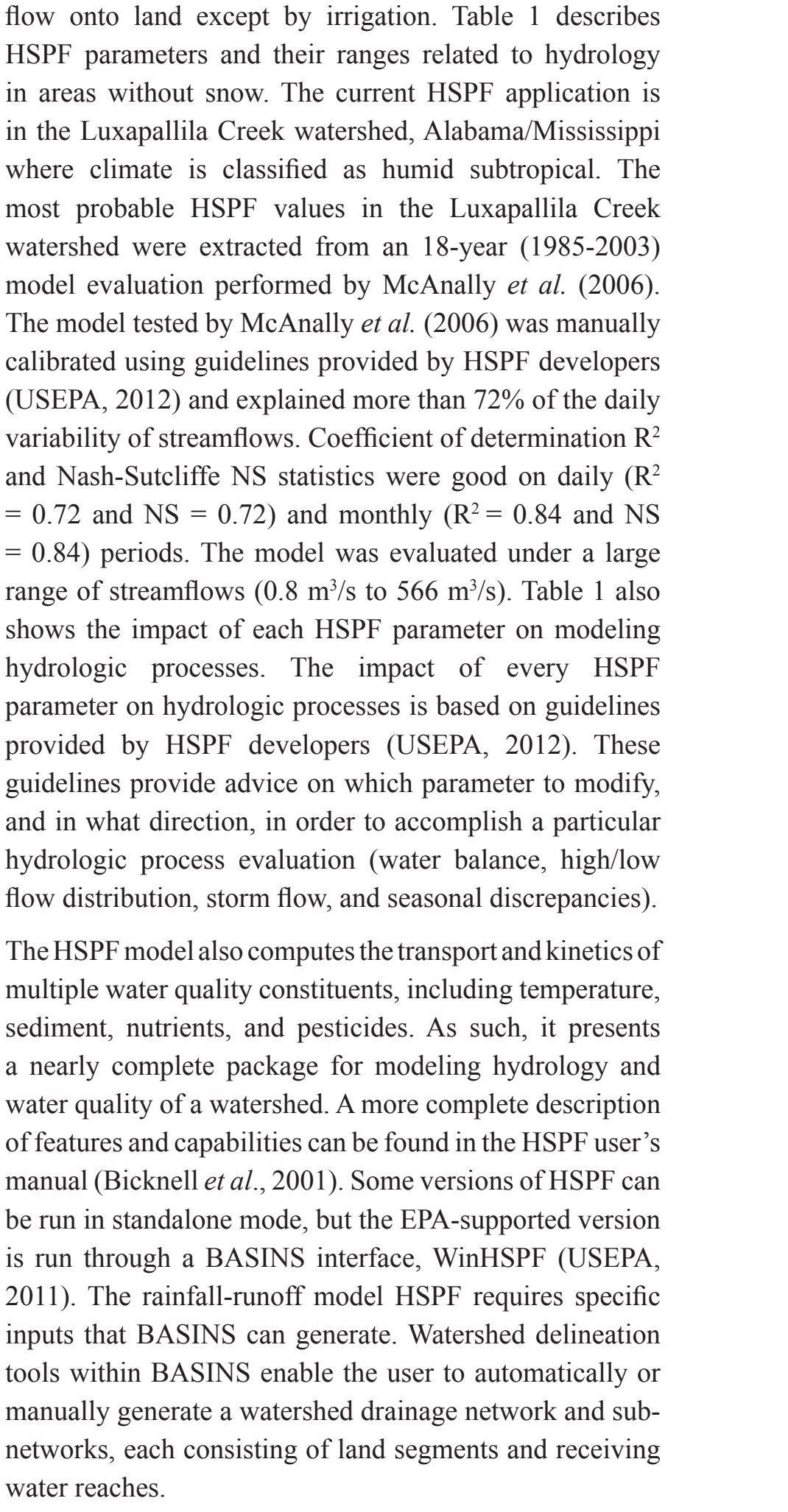

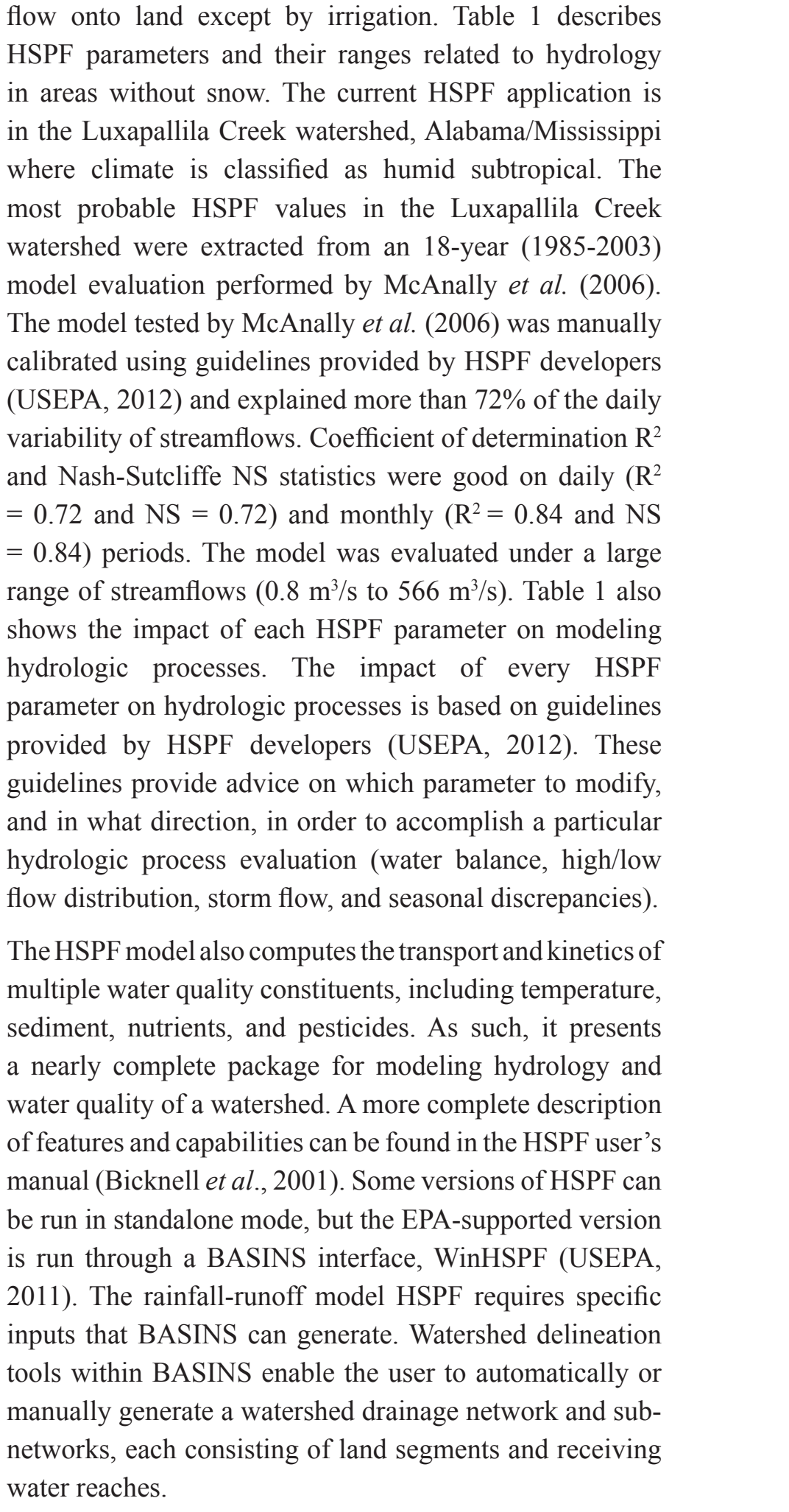

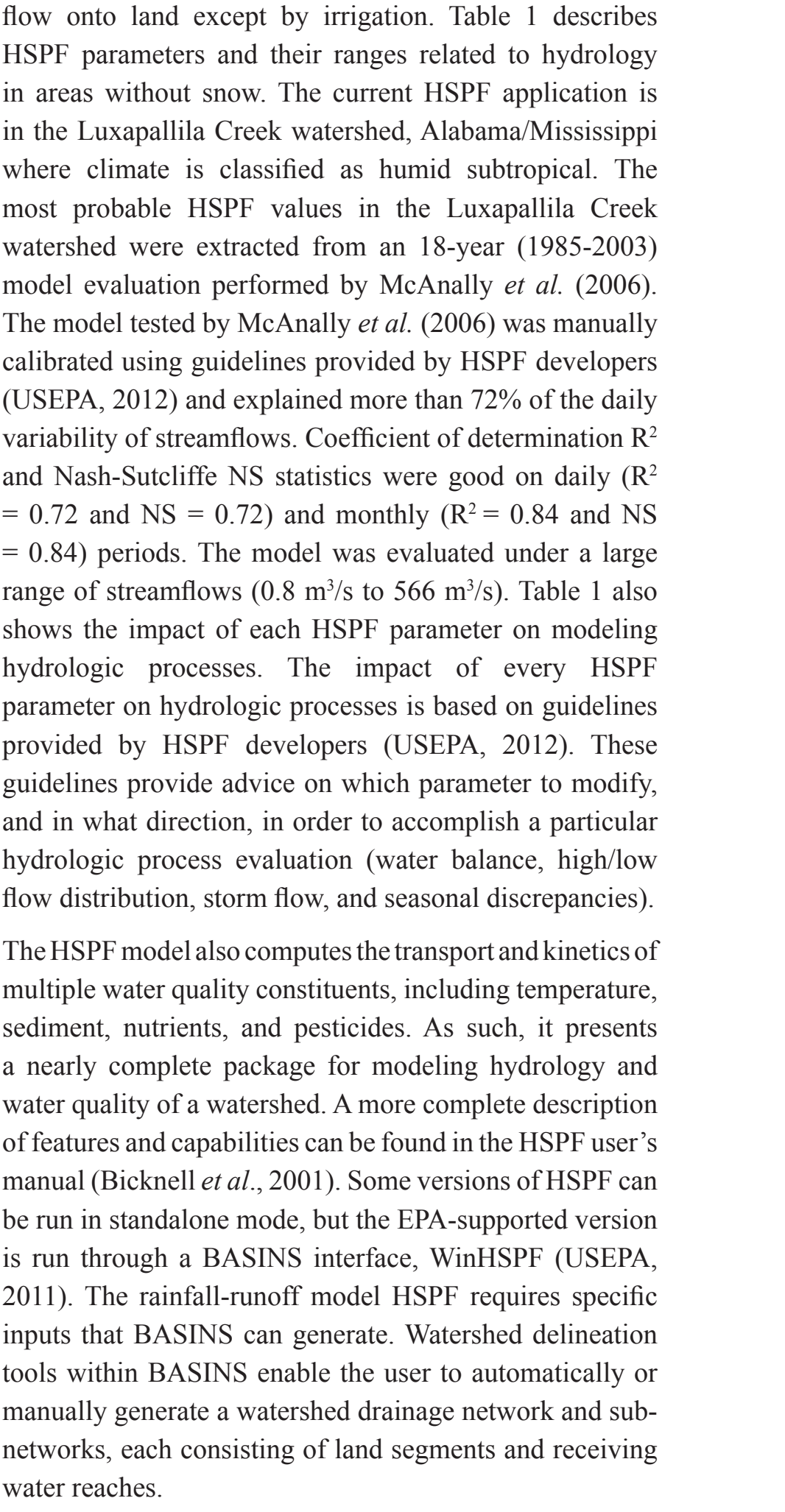

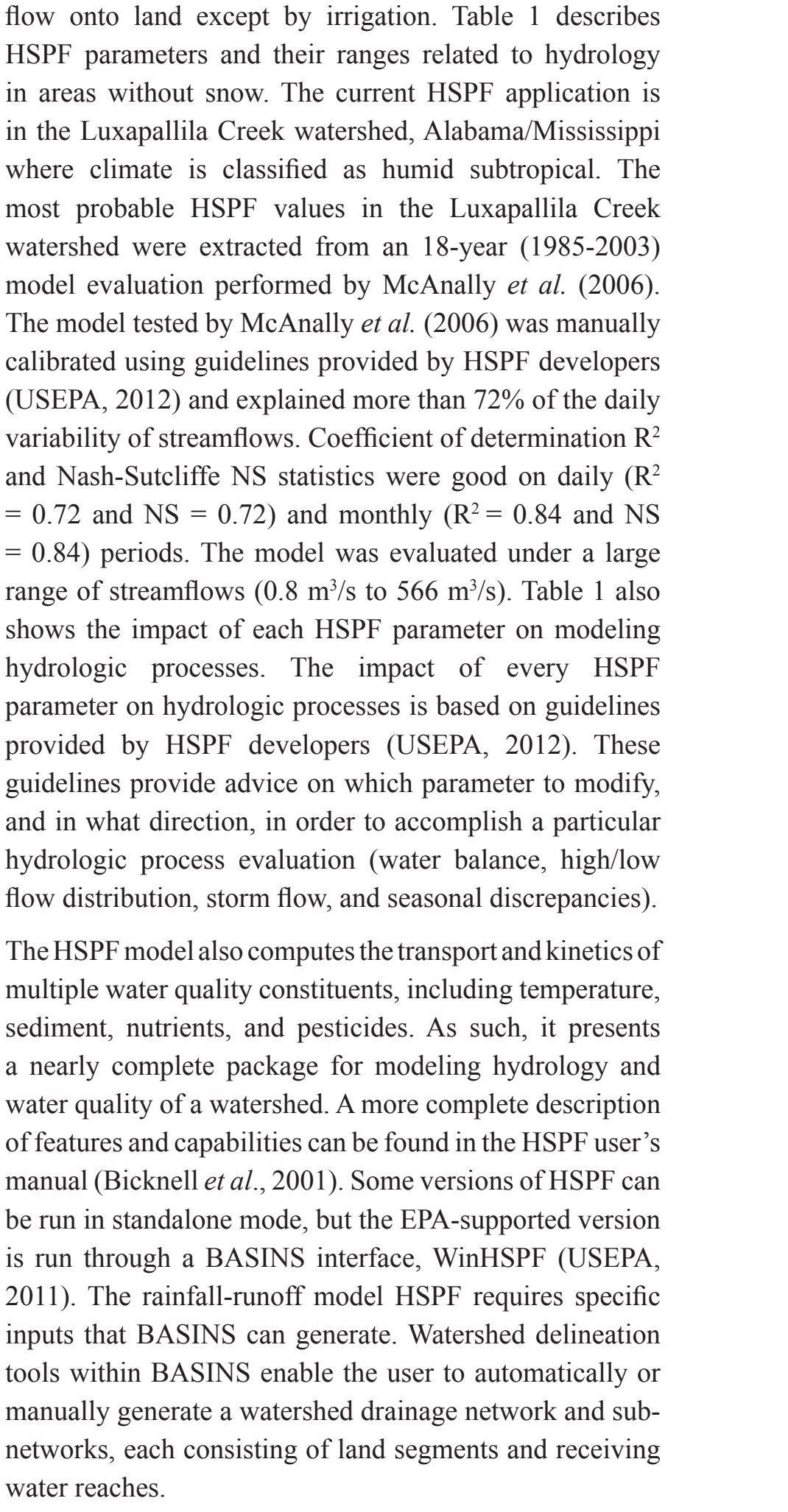

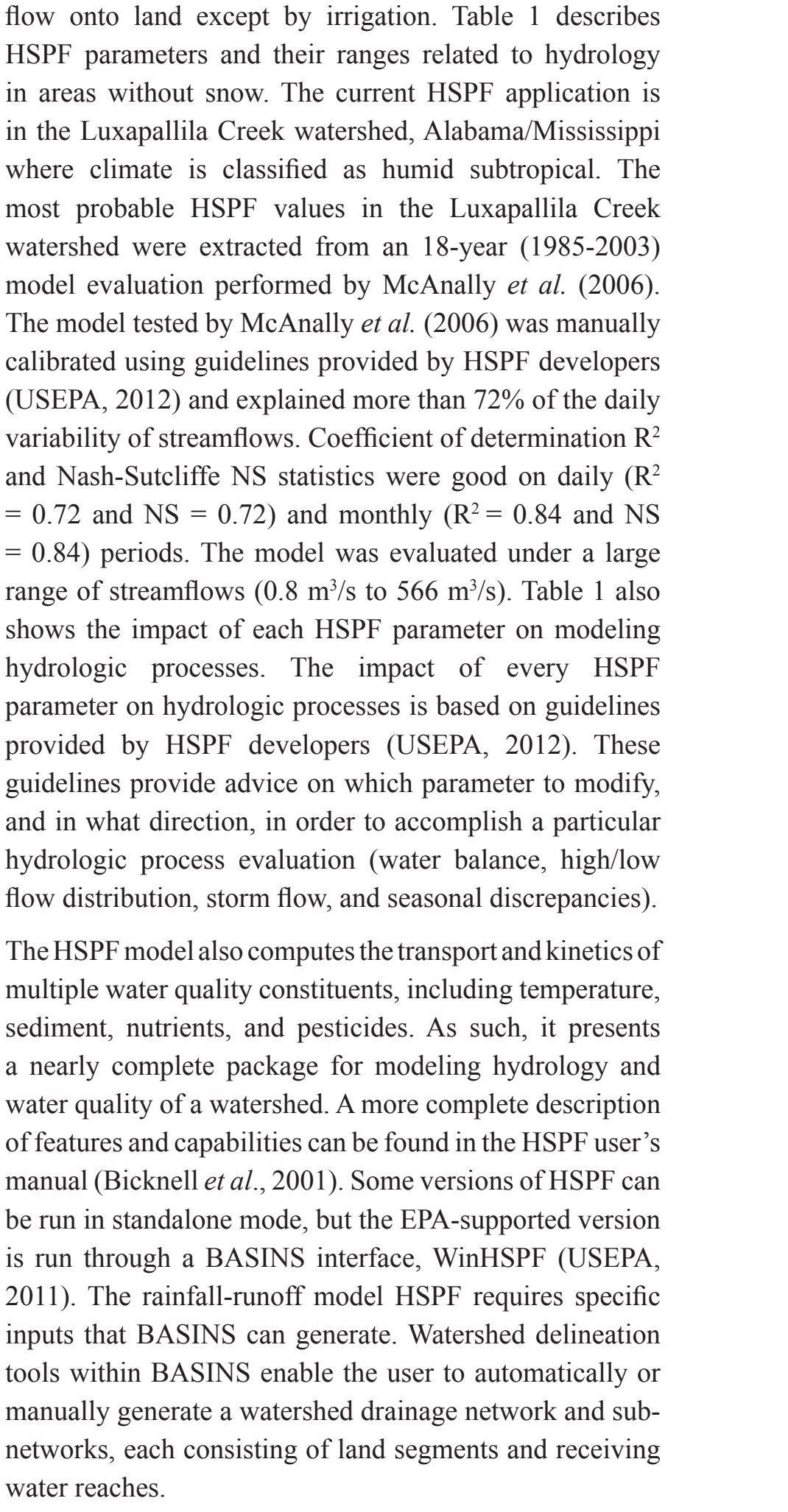

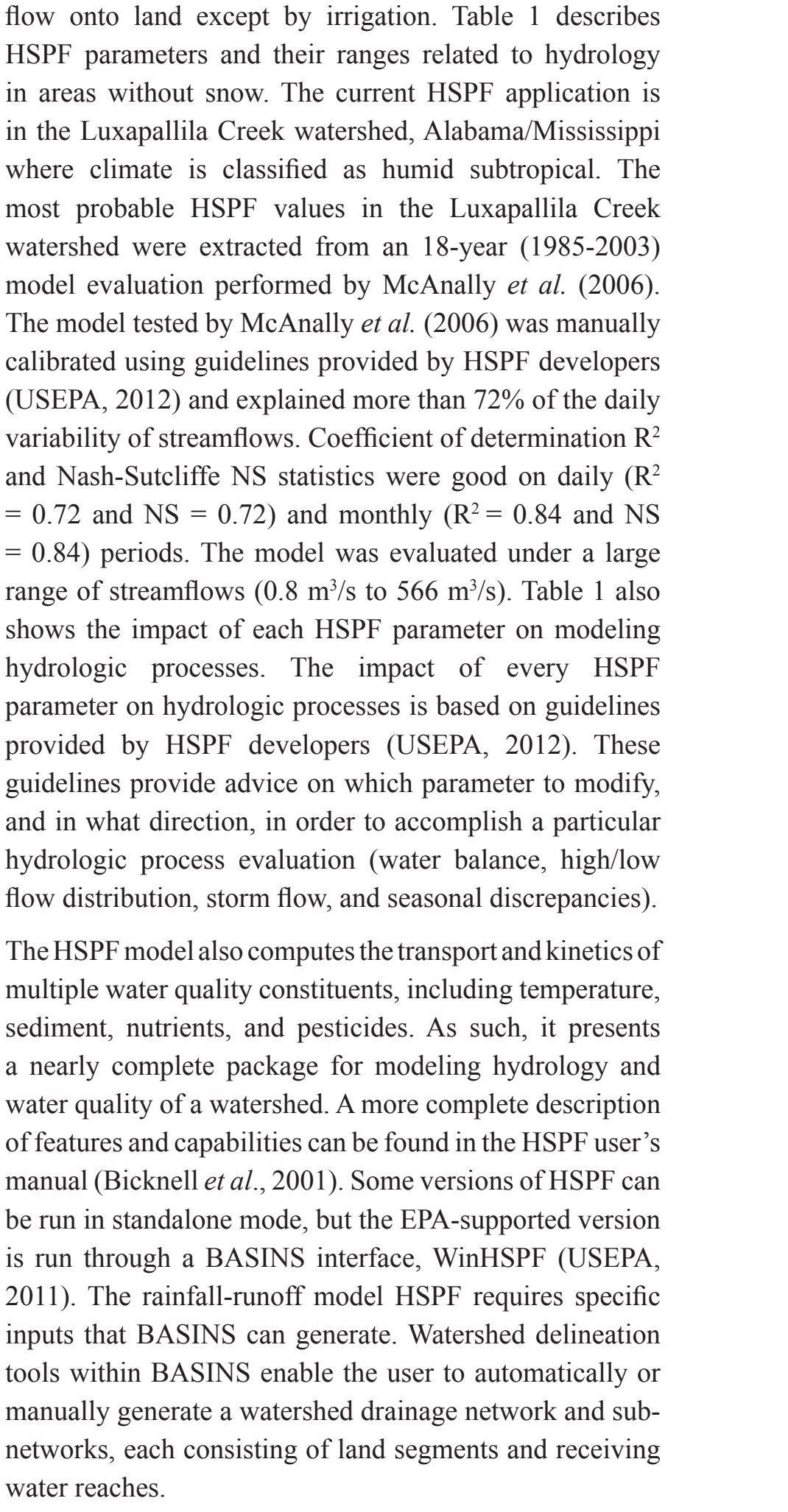

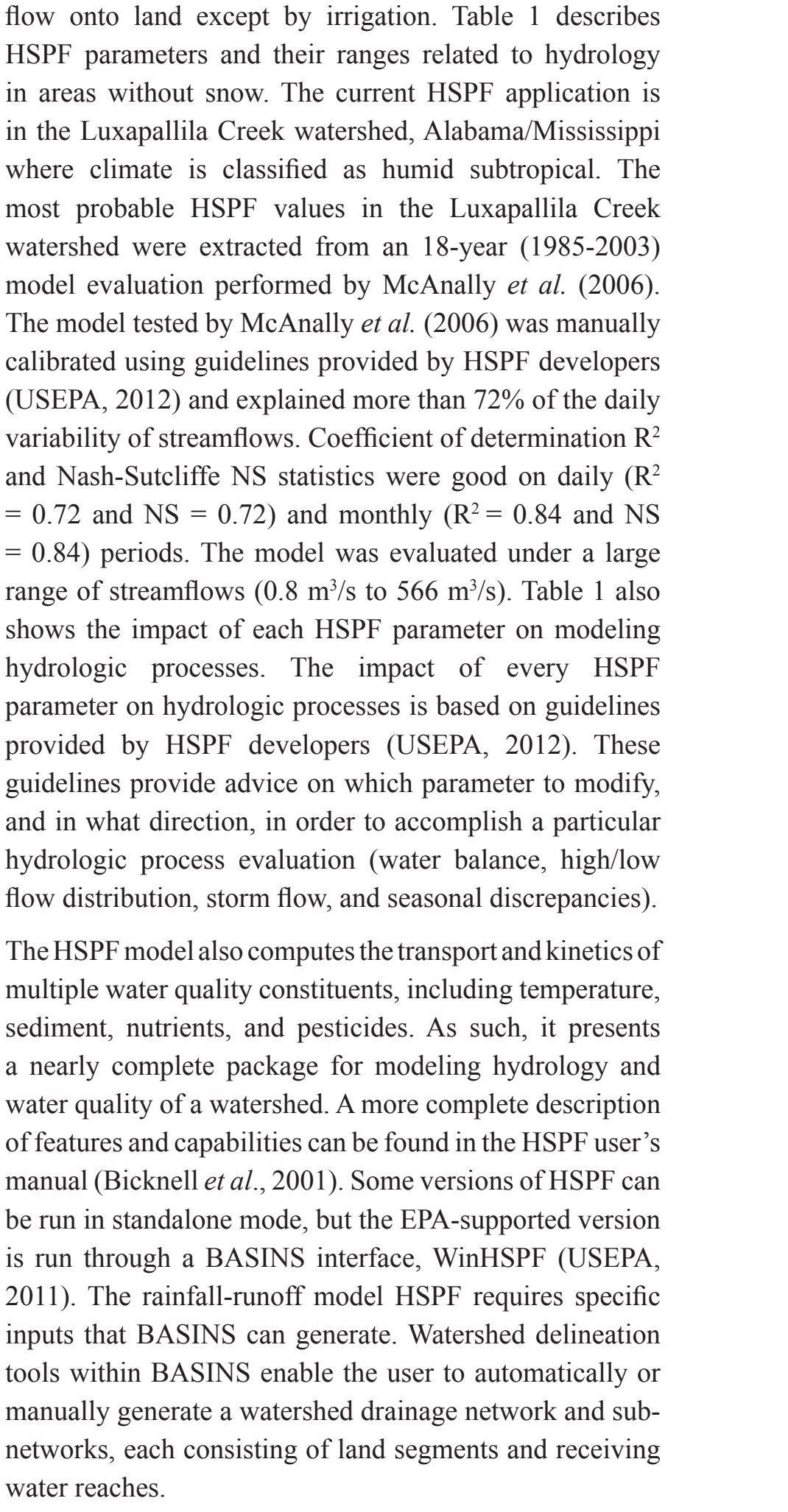

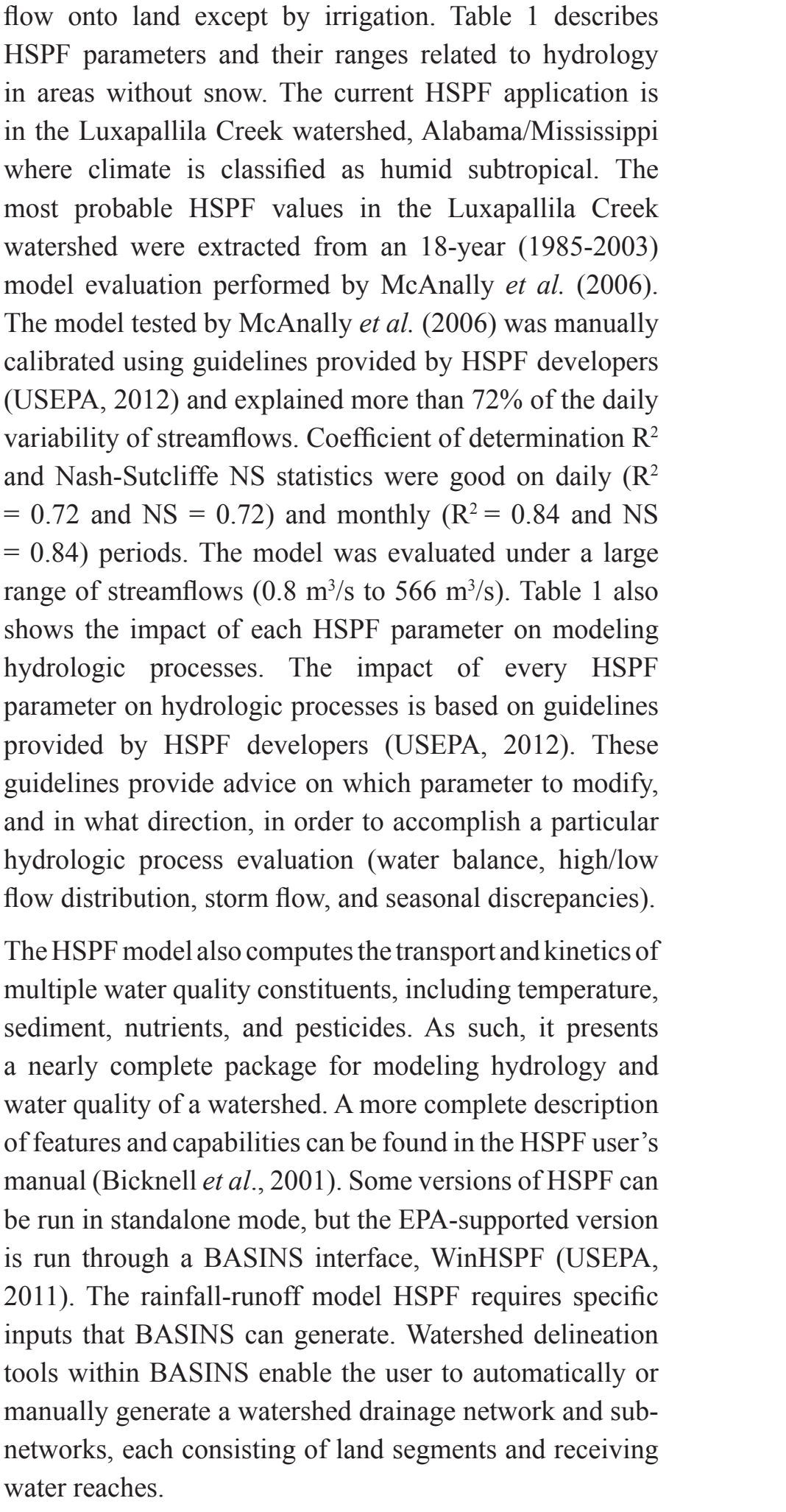

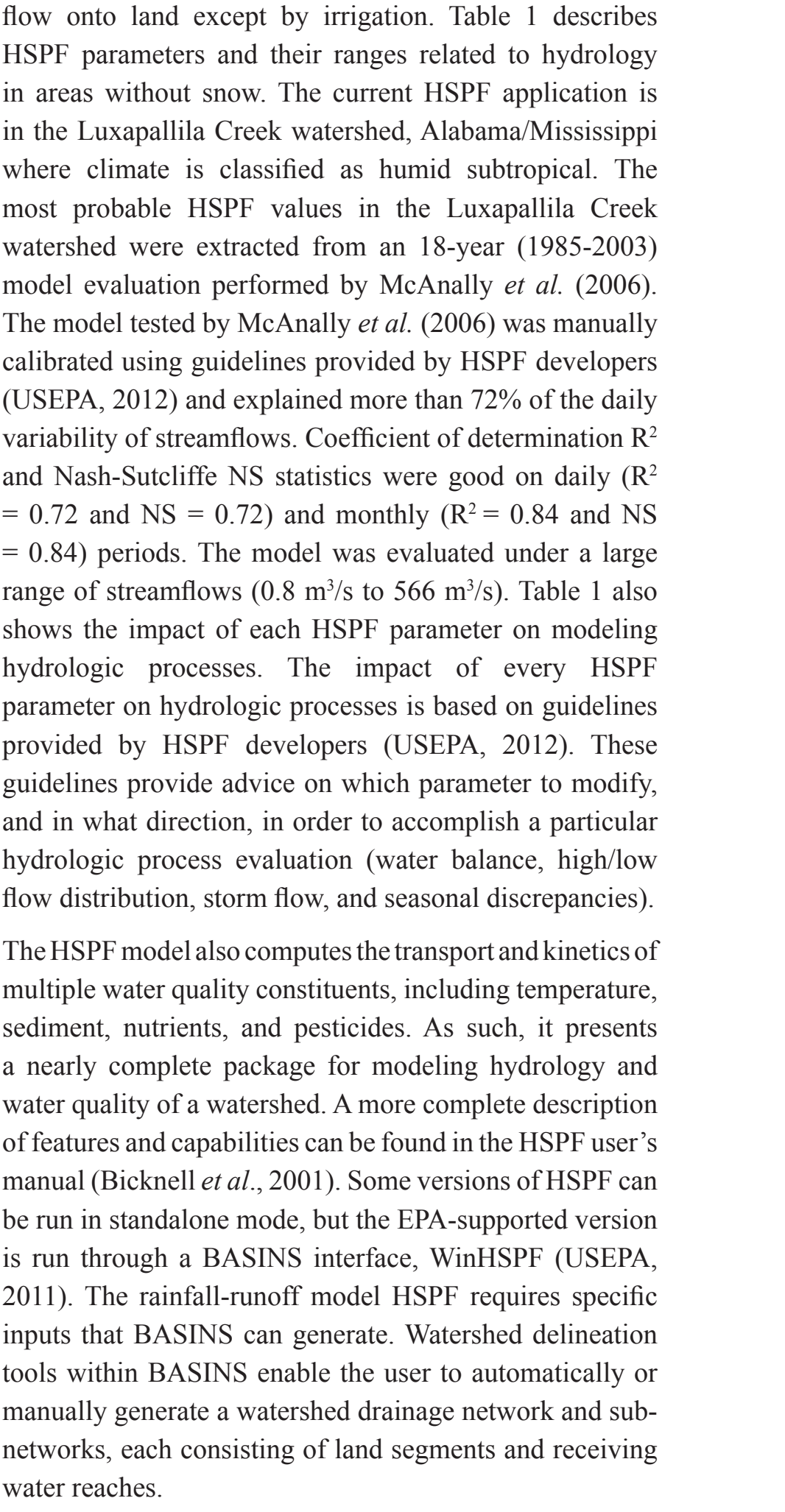

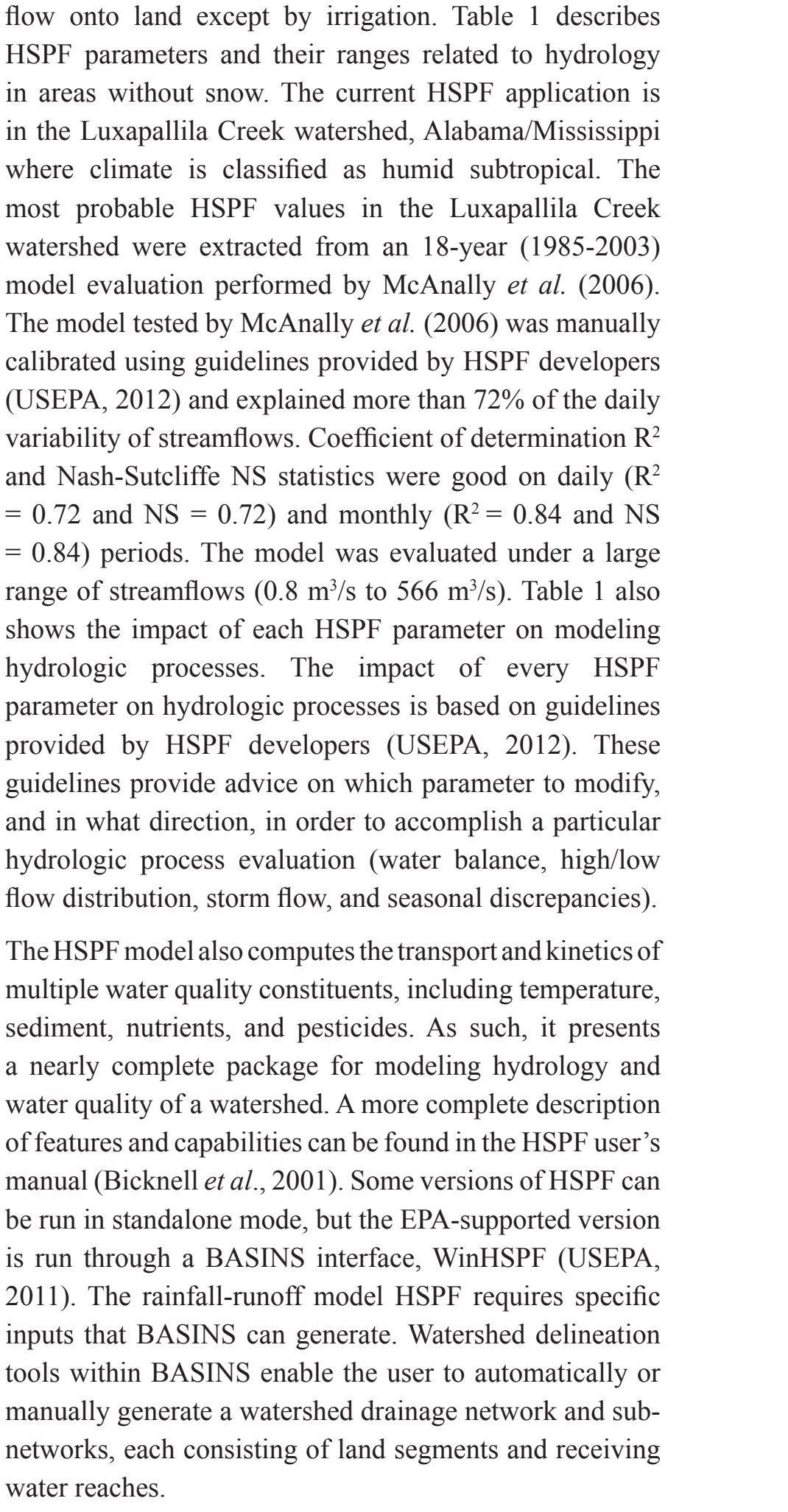

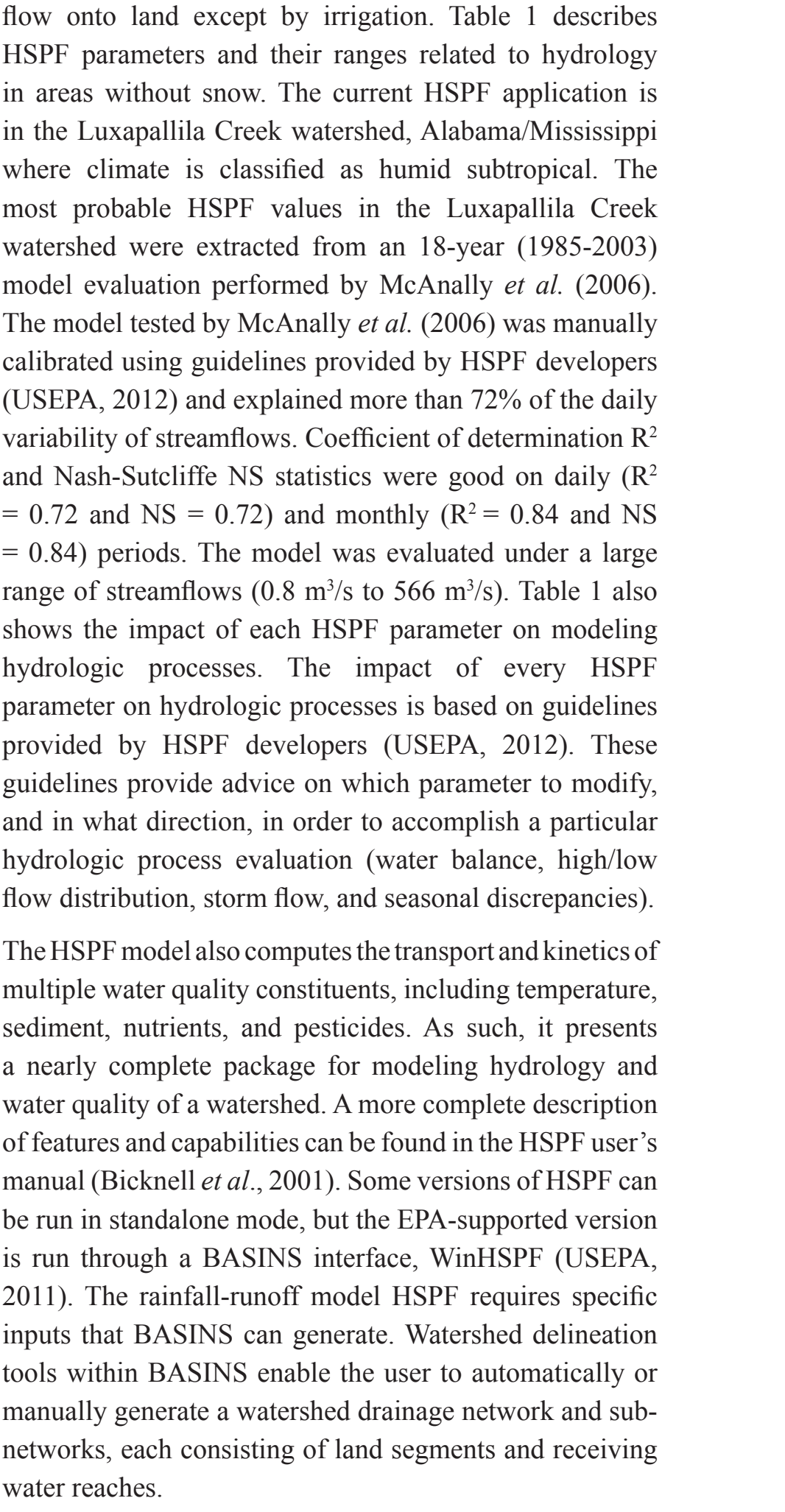

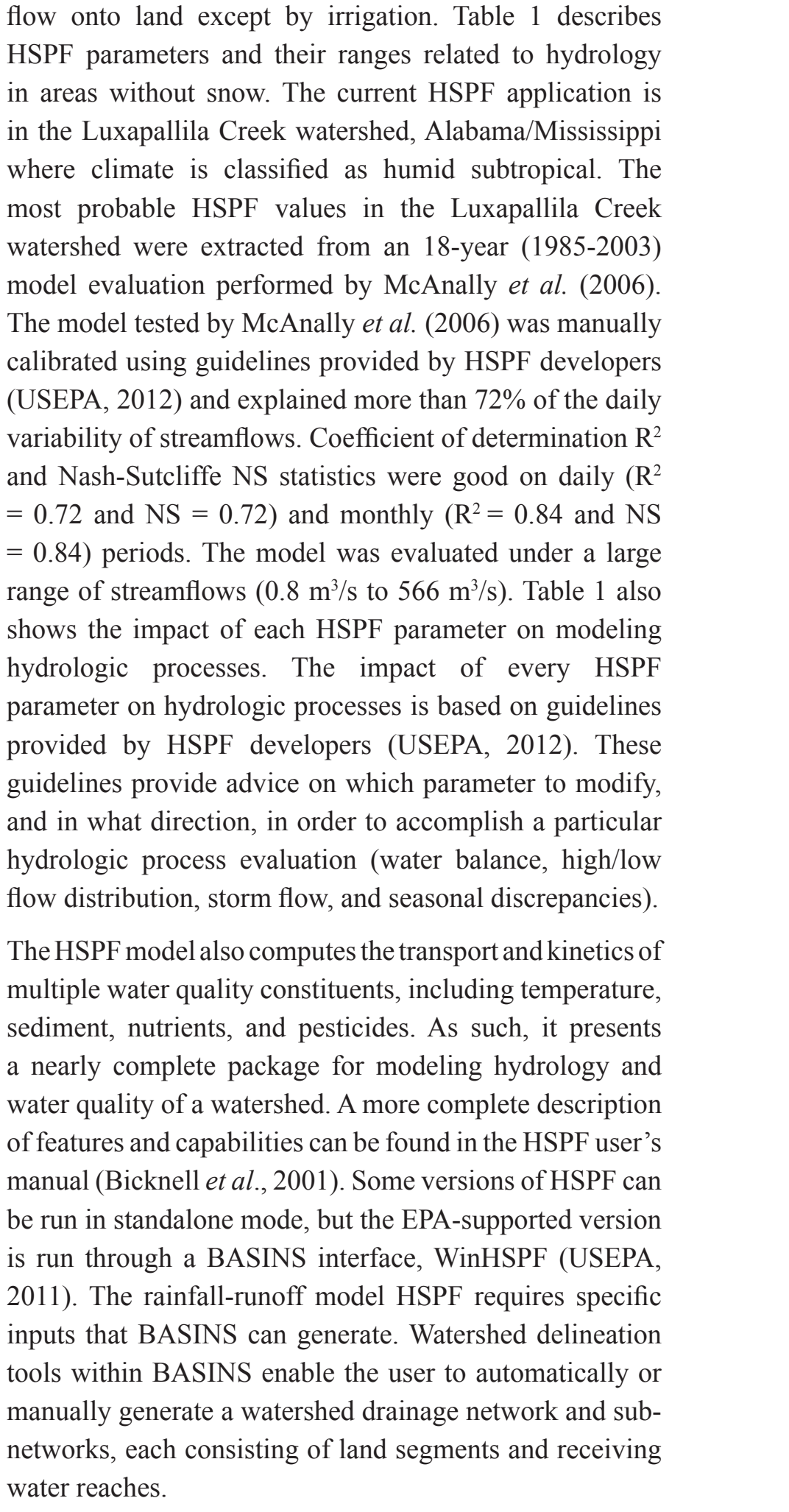

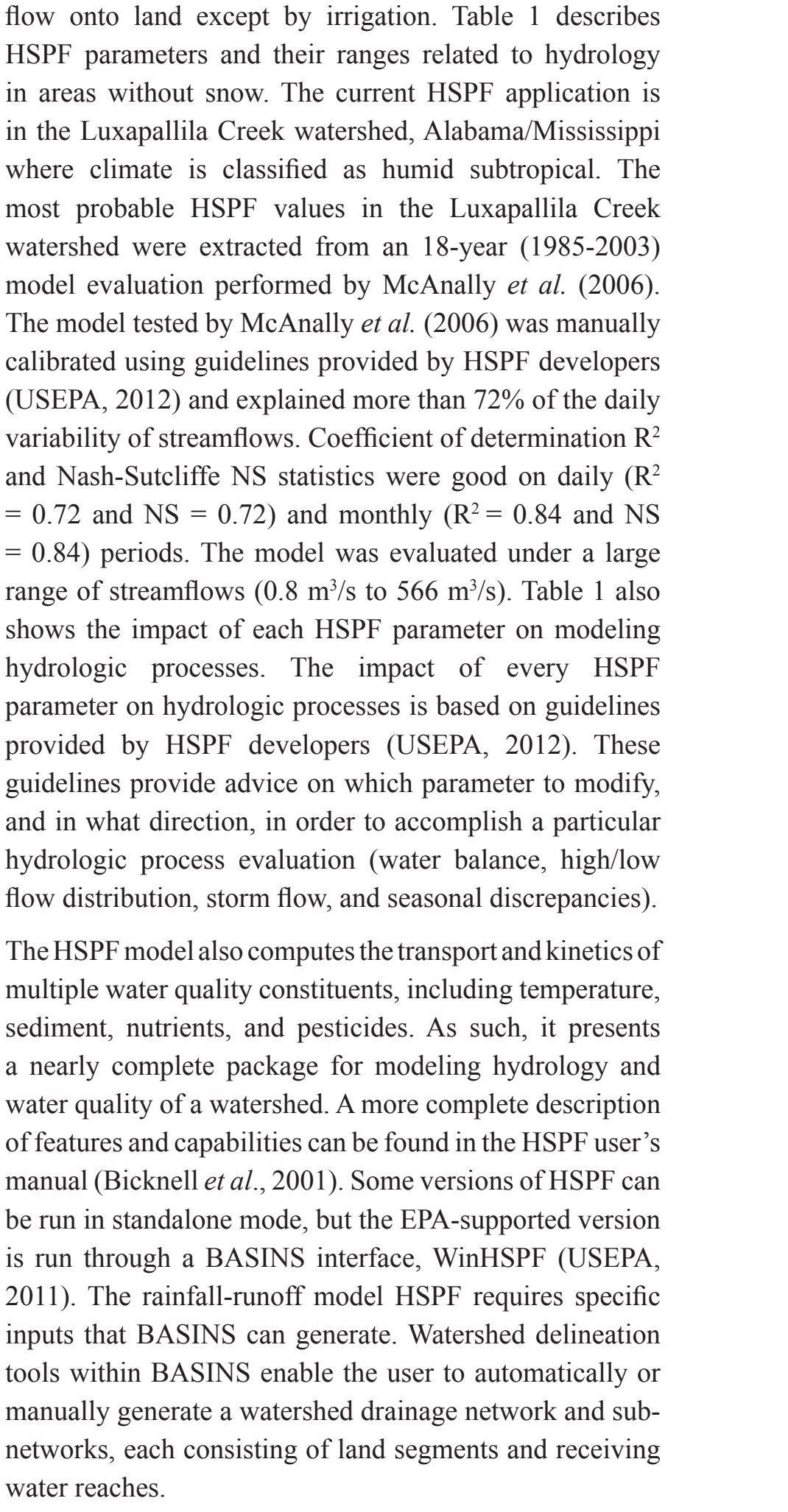

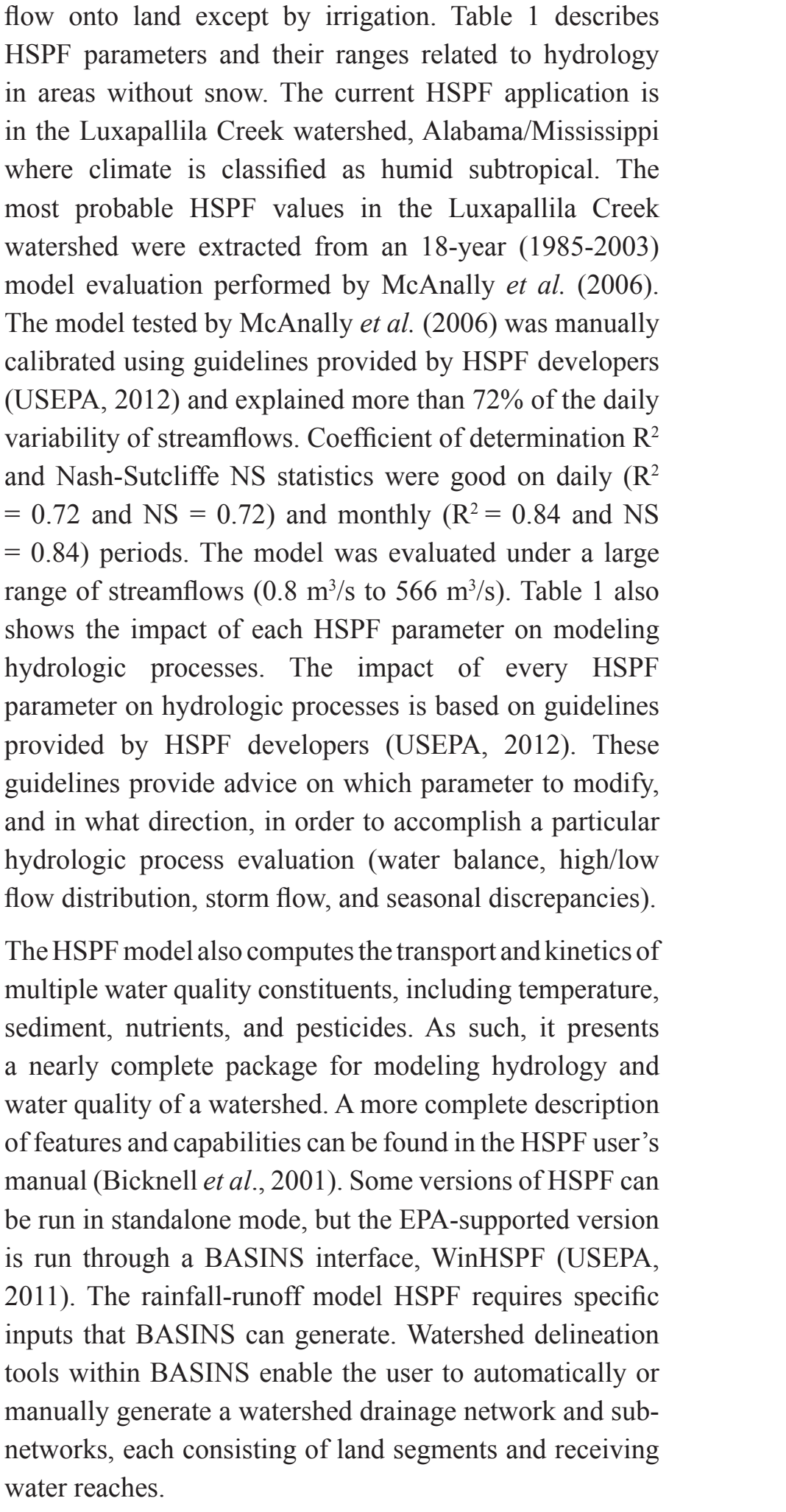

The literature review reports several deterministic applications of the HSPF model (Moore et al., 1988; Laroche et al., 1996; Al-Abed and Whiteley, 2002; Hayashi et al., 2004; Albek et al., 2004; Nasr et al., 2007; Diaz- 
Table 1: HSPF parameter definition and range (USEPA, 2000); most probable value for Luxapallila Creek watershed simulations (McAnally et al., 2006); and hydrologic processes impacted by each parameter marked with X (USEPA, 2012)

\begin{tabular}{|c|c|c|c|c|c|c|c|}
\hline \multirow[b]{2}{*}{ Name } & \multirow[b]{2}{*}{ Definition } & \multirow[b]{2}{*}{ Range } & \multirow{2}{*}{$\begin{array}{l}\text { most } \\
\text { probable } \\
\text { value }\end{array}$} & \multicolumn{4}{|c|}{ Hydrologic Process } \\
\hline & & & & water balance & $\begin{array}{l}\text { high/ } \\
\text { low flow } \\
\text { distribution }\end{array}$ & storm flow & $\begin{array}{c}\text { seasonal } \\
\text { discrepancies }\end{array}$ \\
\hline $\begin{array}{l}\text { LZSN } \\
\mathrm{mm}\end{array}$ & $\begin{array}{l}\text { Lower zone nominal soil } \\
\text { moisture storage }\end{array}$ & $50.8-381.0$ & 228.6 & $\mathrm{x}$ & & & \\
\hline INFILT mm/hr & Index to infiltration capacity & $0.025-12.7$ & 2.8 & $\mathrm{x}$ & $\mathrm{x}$ & $\mathrm{x}$ & \\
\hline $\begin{array}{l}\text { KVARY } \\
1 / \mathrm{mm}\end{array}$ & Variable groundwater recession & $0.0-127.0$ & 45.7 & & & & $\mathrm{x}$ \\
\hline AGWRC & Base groundwater recession & $0.92-0.999$ & 0.997 & & $\mathrm{x}$ & & \\
\hline DEEPFR & $\begin{array}{l}\text { Fraction of groundwater inflow } \\
\text { to deep recharge }\end{array}$ & $0.0-0.5$ & 0.2 & $\mathrm{x}$ & $\mathrm{x}$ & & \\
\hline BASETP & $\begin{array}{l}\text { Fraction of remaining } \\
\text { evapotranspiration from } \\
\text { baseflow }\end{array}$ & $0.0-0.2$ & 0.04 & & $\mathrm{x}$ & & $\mathrm{x}$ \\
\hline AGWETP & $\begin{array}{l}\text { Fraction of remaining } \\
\text { evapotranspiration from active } \\
\text { groundwater }\end{array}$ & $0.0-0.2$ & 0.025 & & & & $\mathrm{x}$ \\
\hline CEPSC mm & Interception storage capacity & $0.0-10.2$ & 3.8 & & & & $\mathrm{x}$ \\
\hline UZSN mm & $\begin{array}{l}\text { Upper zone nominal soil } \\
\text { moisture storage }\end{array}$ & $1.27-50.8$ & 27.9 & & & & $\mathrm{x}$ \\
\hline INTFW & Interflow inflow parameter & $1.0-10.0$ & 3.0 & & & $\mathrm{X}$ & \\
\hline IRC & Interflow recession parameter & $0.3-0.85$ & 0.6 & & & $\mathrm{x}$ & \\
\hline LZETP & $\begin{array}{l}\text { Lower zone evapotranspiration } \\
\text { parameter }\end{array}$ & $0.0-0.9$ & 0.1 & $\mathrm{x}$ & & & $\mathrm{x}$ \\
\hline
\end{tabular}

Ramirez et al., 2008); however few applications attempt to quantify propagation of parameter, input data, and/ or structure uncertainty into model results. Paul (2003) evaluated the effect of parameter uncertainty in the HSPF model to predict in-stream bacterial concentrations using First Order Analysis FOA techniques. He evaluated 10 water quality parameters from pervious and impervious areas and three in-stream water quality parameters. However, he did not evaluate the uncertainty effects of hydrologic/hydraulic parameters on modeling fecal coliform and assumed that the hydrology and hydraulic of the model were well calibrated. Paul (2003) pointed out that water quality parameters from pervious and impervious areas carried on most of the parameter uncertainty in simulated in-stream bacterial concentrations. In particular, the maximum storage of bacteria on pervious land surface parameter contributed with $99.86 \%$ of the variance in simulated peak in-stream concentration of fecal coliform concentration in-stream. The contribution of the three instream water quality parameters to the output variance was negligible $(0.12 \%)$. In addition, he recommended further research to evaluate the effects of hydrology and hydraulic processes on in-stream fecal coliform simulations.

Jia (2004) investigated parameter uncertainties in the HSPF model applying the generalized likelihood uncertainty estimation GLUE approach. A Latin hypercube sampling technique was used to generate random multiple parameter sets. The GLUE method introduced by Beven and Binley (1992) is a Monte Carlo based strategy for evaluation of parametric uncertainty. GLUE accepts multiple sets of parameter values as equal likely representations of a physical system. Other sources of uncertainty such as model structure and input data are treated implicitly within the GLUE framework. Unlike the formal methods for Bayesian inference, GLUE uses "informal" likelihood functions which are formulated without considering the 
structure of the residuals between the observations and the model simulations of a given state variable. Therefore, any measure of goodness of fit such as the Nash and Sutcliffe efficiency criterion, or the total sum of the errors can be implemented in the GLUE methodology (Beven and Binley 1992). Jia (2004) evaluated seven hydrologic parameters at the watershed outlet (i.e. LZSN, INFILT, AGWRC, DEEPFR, UZSN, and IRC). After 50000 HSPF runs, many acceptable parameter sets were identified by the GLUE approach. Information on the total runoff distribution was not available, and wide variations of the total runoff (i.e. surface runoff, interflow, and baseflow) were acceptable.

$\mathrm{Wu}$ (2004) assessed the propagation of parameter uncertainty in both HSPF and CE-QUAL-W2 models using First-Order Error Analysis FOEA. He pointed out that the uncertainty in parameters related to streamflow generation was the main source of variance in simulated nutrient loads. However, when simulated nutrient concentrations were analyzed, some parameters related to hydrology processes have no significant effect. The author justified this difference by the non-linear relationship between pollutant loads and their concentrations. So, FOEA may not be an appropriate method to analyze propagation of parameter uncertainty in complex models. Wu recommends more analysis between FOEA and Monte Carlo analysis.

\section{Harr and Monte Carlo methods}

Uncertainty analysis methods used in hydrology simulation can be arranged in three groups: first-order methods, probabilistic point estimation methods, and Monte Carlo based methods. In this study, the Harr probabilistic point method and Monte Carlo method are used to propagate parameter uncertainty into HSPF streamflow simulations. The concept of probability point estimate methods PPEMs was originated by Rosenblueth (1975). A PPEM propagates the parameter uncertainty by performing point estimations of the function without calculating the derivatives of the function (first-order methods). Selected point estimations of model parameters are calculated using statistical moments (typically the mean and variance) of the variables instead of computing the entire probability density function PDF of the model parameters (as performed by Monte Carlo simulations). Harr (1989) developed a PPEM using the principal component matrix theory. This method considers the mean, standard deviation, and correlation of the parameters. The Harr method propagates the parameter uncertainty through model outputs by performing two point estimations of the parameter space. The correlation matrix of parameters, $C$, is decomposed as

$$
C=e \lambda e^{T}
$$

where $e$ is the eigenvector matrix; $\lambda$ is the diagonal eigenvalue matrix and $e^{T}$ is the transpose of the eigenvector matrix. Thus, someone using Harr's method must generate the correlation matrix of selected parameters and then compute, using mathematical programs such as MATLAB, the eigenvector matrix and the diagonal eigenvalue matrix. The uncorrelated and standardized coordinates can be calculated by

$$
\begin{aligned}
& x_{i}^{+}=\mu+\sqrt{n} \sigma e_{i} \\
& x_{i}^{-}=\mu+\sqrt{n} \sigma e_{i}
\end{aligned}
$$

where $\mu$ is the vector of the expected values of the parameter; $n$ is the number of parameters; $\sigma$ is the diagonal matrix of the standard deviation of the parameters; and $e_{i}$ is the eigenvalue $\lambda_{i}$. Finally, based on the two coordinates selected along each eigenvector (2a) and (2b), the user must compute the corresponding model output values. For instance, this research used 12 HSPF parameters; thus 24 coordinates were calculated and 24 model outputs were generated for each simulated day. Then, the 95th and 5th percentiles of these 24 model outputs were calculated to generate the $90 \%$ uncertainty bounds of model outputs using the percentile function in MATLAB. As a summary, the Harr method involves the following steps:

1. Identify model parameter ranges and sample values of each parameter;

2. verify the symmetry of each input parameter (if the distribution is not symmetric, the Harr method is not appropriate. However, parameter transformation could be performed to ensure input parameters are symmetric);

3. compute mean and standard deviation of each parameter; 
4. calculate the correlation matrix of each parameter;

5. determine eigenvectors from the correlation matrix;

6. compute $2 n$ coordinate points using equations ( $2 a)$ and $(2 b)$;

7. evaluate the model with parameter values computed in step 6;

8. analyze the model outputs (percentiles, mean, standard deviation, etc).

A drawback of the Harr method is that the uncorrelated and standardized coordinates may fall outside the parameter bounds (Christian and Baecher, 2002). In this study, when a coordinate was outside the pre-established HSPF parameter range, the closest parameter limit was used instead of the outside value. This issue can be related to poor definition of model parameter range. However, HSPF hydrologic algorithms have been tested since 1960 and model developers have developed a comprehensive list of parameter ranges (USEPA, 2000). An application of the Harr method in simulating watershed hydrology is found in Yu et al. (2001).

The Monte Carlo method computes an empirical probability distribution of the model output using random values for the input variables sampled from their probability distribution (Metropolis and Ulam, 1949). Detailed information on Monte Carlo simulation is found in Ronen (1988), Morgan and Henrion (1990), and Sobol' (1994). The Monte Carlo simulation is the best known uncertainty method, and the simplest way of sampling the entire range of likely observations of the system being studied (Morgan and Henrion, 1990). Melching (1995) declared that the Monte Carlo method "may be the only method that can estimate the cumulative density function CDF and PDF of $\mathrm{Z}$ (a model parameter) for cases with highly nonlinear and/or complex system relationships." The Monte Carlo simulation involves five steps:

1. Generate probability distributions of selected model parameters (e.g., normal, triangular, beta, etc.);

2. calculate a random value from the parameter's distributions;

3. evaluate the model using the random value calculated in step 2;
4. repeat steps 2 and 3 many times; and

5. analyze the model outputs (e.g., $\mathrm{CDF}$, percentiles, mean, standard deviation, etc.).

The Monte Carlo simulation has been applied to study the uncertainty of forcing input data and model parameters in computer models of watershed hydrology (Melching, 1995; Carpenter and Georgakakos, 2004). Melching (1995) stated that "for complex, nonlinear models with many uncertainty basic variables, however, the number of simulations (thus the computer time) necessary to achieve an accurate estimate may become prohibitive." Increasing of computer processing speeds makes computations more tractable. Monte Carlo method results have been used as a baseline when comparisons with other uncertainty methods have been done (Binley et al., 1991; Melching, 1992; Melching, 1995; Yu et al., 2001).

In summary, the Harr method is computationally more efficient than the Monte Carlo method. In Harr's method, mean, standard deviation of parameters and their correlations are used to propagate parameter uncertainty into model results. The Harr method is limited to symmetrical distributions and sometimes the uncorrelated and standardized coordinates are calculated out of the parameter bounds. The computation algorithm of the Monte Carlo method has a simple structure and is used in complex and nonlinear models. In the Monte Carlo method, random parameter inputs are computed from their probability distributions and are then propagated through model results. The Monte Carlo method is computationally time consuming at high levels of accuracy.

\section{Methodology \\ Study area}

This study used physical data from the Luxapallila Creek watershed located in the Southeastern of United States. The watershed flows through Fayette, Lamar, Marion, and Pickens counties in Alabama and into Lowndes and Monroe counties in Mississippi (Figure 1). Near the outlet (USGS Station 02443500), the watershed has a drainage area of $1.801 \mathrm{~km}^{2}$, an average basin slope of $2 \%$, and average annual precipitation (1982 - 2004) of $1.379 \mathrm{~mm}$ recorded at the Millport 2E weather station. Seasonal fluctuations in rainfall result in maximum river discharges 
from January to April and minimum discharges from August to September. Elevation in the study area ranges from 45 to $274 \mathrm{~m}$ mean sea level. The USGS Geographic Information Retrieval and Analysis System GIRAS, states that land cover developed in the early 1980's is distributed as $73 \%$ forest land, $20 \%$ agricultural land, $6 \%$ wetlands, and $1 \%$ other land types (barren, urban, and non-urban). More information about the Luxapallila Creek watershed can be found at Diaz-Ramirez et al. (2011).

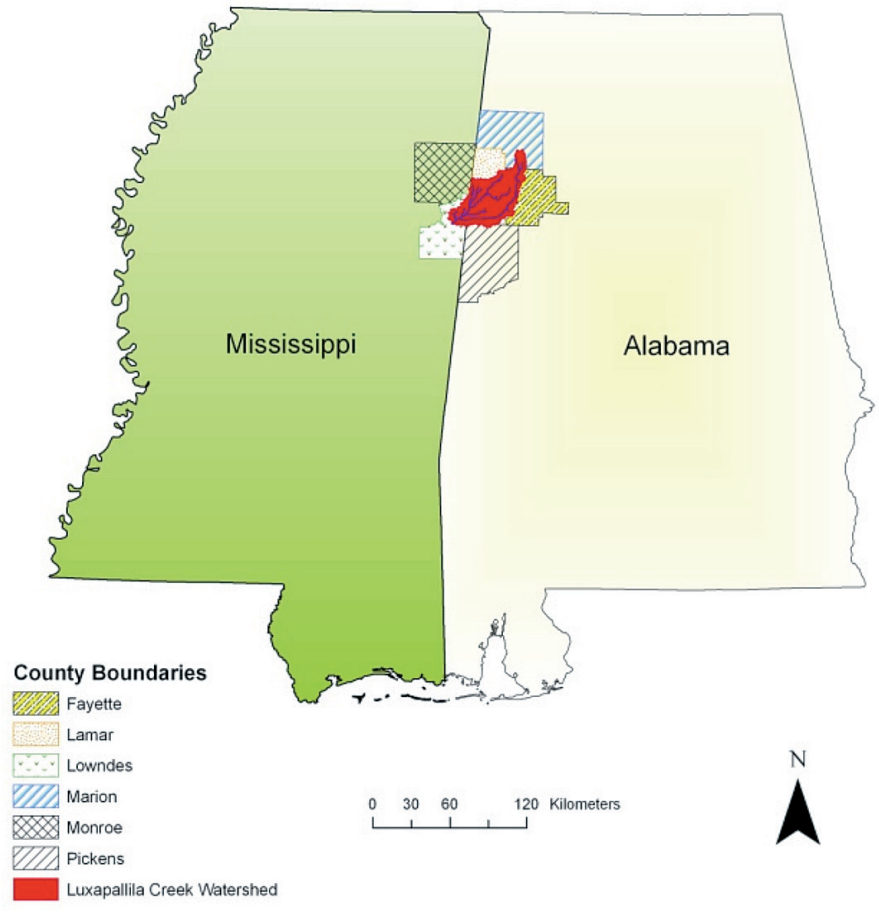

Figure 1: Location of the Luxapallila Creek watershed

\section{HSPF model set up}

The Luxapallila watershed model was set up with a standard set of procedures and data as might be used in any BASINS application to provide a HSPF input data file (uci file). Spatial and climatic time series databases, including land use, overland flow slope and length, reach characteristics, and detailed meteorological data are used as inputs to HSPF. The model was lumped using one basin area and one main channel because streamflow-gauging station data from only one station were available (USGS 2443500). Topographic data were created from the standard USGS Digital Elevation Models DEMs, and the DEMs were also used to delineate the watershed boundaries. The length and slope of overland flow and reach were calculated and kept constant throughout the simulations. Manning's $n$ roughness coefficients for overland flows were determined by literature review and were kept constant throughout the simulations. The watershed was partitioned into five pervious and one impervious land types (Table 2).

Table 2: Pervious and impervious land types simulated using 1980 GIRAS data

\begin{tabular}{lcc}
\hline \multicolumn{1}{c}{ Land cover } & $\begin{array}{c}\text { Surface area } \\
\mathrm{km}^{2}\end{array}$ & $\begin{array}{c}\text { Surface area } \\
\%\end{array}$ \\
\hline Forest land & 1316.9 & 73.1 \\
Agricultural land & 360.0 & 20.0 \\
Barren land & 2.5 & 0.1 \\
Wetlands & 104.4 & 5.8 \\
Urban land (pervious) & 8.1 & 0.4 \\
Urban land (impervious) & 8.1 & 0.4 \\
Water & 1.5 & 0.1 \\
\hline
\end{tabular}

Hourly precipitation data were NEXRAD stage IV data from the Earth Observing Laboratory web page (http://data. eol.ucar.edu/codiac/dss/id=21.093). Downloaded rainfall data were uncompressed and incorporated into input files by use of the Watershed Data Management WDMUtil software (Hummel et al., 2001). Hourly potential evapotranspiration, air temperature, dew point, wind speed, solar radiation, evaporation, and cloud cover values were obtained from the Haleyville station. The weather database for the Haleyville station was downloaded from the BASINS web site. The model was run for data from 01/01/2002 through 12/31/2005. The model time step was hourly, but streamflow data were output daily to compare with observed data (USGS Station 02443500).

\section{Computational experiment}

\section{Monte Carlo method}

The first step in the Monte Carlo simulation MCS was to determine the probability density functions PDFs for the input parameters considered in the study. In most studies this is performed by using a non-informative uniform distribution for each parameter, which covers a feasible range of parameter values for the particular study. Due to the lack of data to estimate the PDFs, all parameters were assigned a triangular distribution, which is defined by the lowest, most probable, and highest values. Most probable values were extracted from an 18-year model calibration of the Luxapallila Creek watershed (McAnally et al., 2006), see Table 1. Highest and lowest values were 
assigned based on the EPA BASINS Technical Note 6 (USEPA, 2000), also in Table 1. Haan (2002) pointed out that the accuracy of the Monte Carlo simulations is a function of the assumed PDF and number of simulations performed. In selecting PDFs and number of simulations, there is no defined answer and judgment is required to make these decisions (Haan, 2002). Authors believe that taking into consideration the calibrated parameters (most probable values) from a long term deterministic evaluation (McAnally et al., 2006) in the study area will positively impact the Monte Carlo method results by forcing the parametric space search around the most probable values.

Five thousand random samples from the $12 \mathrm{HSPF}$ parameter's distributions (triangular distributions) were generated using MATLAB. Then, the HSPF program was run using the selected random samples from 01/01/2002 to $12 / 31 / 2005$. Finally, evaluation of streamflow simulations was accomplished (stability results, 95 th and 5 th percentiles) at daily levels with 5.000 streamflow simulations for each simulation day.

To determine the number of realizations (stability results) sufficient to analyze the uncertainty of streamflow simulations, the values of Absolute Relative Errors ARE of simulated daily flows were calculated as

$$
A R E=\sum_{i=1}^{N}\left[\frac{\left|Q_{i+1}-Q_{i}\right|}{Q_{i}}\right]
$$

where $N$ is the number of Monte Carlo simulations; and $Q_{i}$ is the simulated daily flow for run $i$. For instance, in this study, 1.096 daily HSPF streamflows from 01/01/2003 through 12/31/2005 were used; this means that $1.095 \mathrm{ARE}$ results were calculated for each Monte Carlo simulation.

\section{Harr method}

The first step in the Harr method was to calculate the correlation matrix, mean, and standard deviation of the 12 HSPF parameters evaluated. The USEPA developed a database of HSPF model parameters (USEPA, 2006). This database was called HSPFParm and contains HSPF parameter values of several model applications in the U.S. Twenty seven sets of parameter values were used to compute the correlation matrix, mean, and standard deviation of selected parameters. Table 3 depicts mean, standard deviation, median, mode, and skew values of selected HSPF parameters. In a symmetric distribution, the mean, median, and mode are the same (Haan, 2002). For each parameter in Table 3, it can be observed that these three statistically measured values are close and the skew values are around zero. This means that the assumption of symmetry for input distributions in the Harr's method is most likely valid in this study. Other uncertainties could arise in using the Harr's method. For example, the short sets of parameter values (only 27) and the lack of parameter values found in the study site or near watersheds. Table 4 shows the correlation matrix of selected HSPF parameters. Then, the eigenvector and eigenvalue matrices from the correlation matrix were calculated using MATLAB.

Table 3: Statistical measure values of selected HSPF parameters

\begin{tabular}{lccccc}
\hline \multicolumn{1}{c}{ Parameter } & mean & $\begin{array}{c}\text { standard } \\
\text { deviation }\end{array}$ & median & mode & skew* \\
\hline LZSN, mm & 146.7 & 48.0 & 156.4 & 180.6 & -0.6 \\
INFILT, mm/hour & 2.0 & 1.0 & 1.9 & 1.9 & 1.6 \\
KVARY, 1/mm & 24.3 & 25.4 & 24.8 & 0.0 & 0.5 \\
AGWRC & 0.97 & 0.02 & 0.98 & 0.99 & -1.5 \\
DEEPFR & 0.04 & 0.1 & 0.002 & 0.00 & 3.9 \\
BASETP & 0.02 & 0.02 & 0.02 & 0.00 & 1.5 \\
AGWETP & 0.02 & 0.04 & 0.02 & 0.00 & 2.1 \\
CEPSC, mm & 0.3 & 0.7 & 0.00 & 0.00 & 2.1 \\
UZSN, mm & 14.8 & 7.3 & 10.9 & 10.8 & 0.8 \\
INTFW & 2.9 & 1.7 & 2.7 & 2.7 & 1.7 \\
IRC & 0.7 & 0.2 & 0.8 & 0.8 & -2.1 \\
LZETP & 0.3 & 0.3 & 0.3 & 0.0 & 0.5 \\
\hline
\end{tabular}

* dimensionless

The model runs required to solve the system were 2 by the number of parameters. In this study, 12 parameters were evaluated; thus 24 model HSPF runs were required to solve the system. Using equations (2a) and (2b), the coordinates of the 24 intersection points by each HSPF parameter were calculated. Coordinate values out of range were changed by the closest limit value. Finally, using these 24 sets of parameters to determine the 95th and 5th percentiles of model outputs, the $90 \%$ uncertainty bounds (95th-5th percentiles) were calculated at daily levels from $01 / 01 / 2003$ to $12 / 31 / 2005$.

\section{Performance evaluation}

The overall effect of parameter uncertainty on streamflow 
Table 4: Correlation matrix of selected HSPF parameters

\begin{tabular}{lcccccccccccc}
\hline & LZSN & INFILT & KVARY & AGWRC & DEEPFR & BASETP & AGWETP & CEPSC & UZSN & INTFW & IRC & LZETP \\
\hline LZSN & 1.0 & 0.1 & 0.6 & -0.2 & -0.1 & 0.2 & 0.3 & 0.0 & 0.4 & 0.1 & -0.3 & 0.4 \\
INFILT & 0.1 & 1.0 & 0.1 & 0.2 & 0.5 & -0.1 & 0.1 & 0.6 & 0.1 & 0.0 & -0.2 & 0.3 \\
KVARY & 0.6 & 0.1 & 1.0 & -0.3 & 0.0 & 0.3 & 0.5 & -0.3 & 0.6 & 0.3 & -0.2 & 0.6 \\
AGWRC & -0.2 & 0.2 & -0.3 & 1.0 & 0.1 & -0.6 & -0.2 & 0.1 & 0.0 & -0.1 & -0.1 & -0.5 \\
DEEPFR & -0.1 & 0.5 & 0.0 & 0.1 & 1.0 & -0.1 & 0.1 & 0.0 & -0.1 & -0.3 & -0.2 & 0.0 \\
BASETP & 0.2 & -0.1 & 0.3 & -0.6 & -0.1 & 1.0 & 0.7 & 0.2 & 0.0 & -0.1 & -0.2 & 0.2 \\
AGWETP & 0.3 & 0.1 & 0.5 & -0.2 & 0.1 & 0.7 & 1.0 & 0.3 & 0.1 & -0.1 & -0.5 & 0.1 \\
CEPSC & 0.0 & 0.6 & -0.3 & 0.1 & 0.0 & 0.2 & 0.3 & 1.0 & 0.0 & -0.1 & -0.5 & 0.0 \\
UZSN & 0.4 & 0.1 & 0.6 & 0.0 & -0.1 & 0.0 & 0.1 & 0.0 & 1.0 & 0.4 & -0.2 & 0.4 \\
INTFW & 0.1 & 0.0 & 0.3 & -0.1 & -0.3 & -0.1 & -0.1 & -0.1 & 0.4 & 1.0 & 0.2 & 0.6 \\
IRC & -0.3 & -0.2 & -0.2 & -0.1 & -0.2 & -0.2 & -0.5 & -0.5 & -0.2 & 0.2 & 1.0 & 0.1 \\
LZETP & 0.4 & 0.3 & 0.6 & -0.5 & 0.0 & 0.2 & 0.1 & 0.0 & 0.4 & 0.6 & 0.1 & 1.0 \\
\hline
\end{tabular}

simulations was evaluated by computing the 5 th and 95 th percentiles (i.e. 90\% uncertainty bounds) of the Monte Carlo and Harr results. Two criteria were used to evaluate the HSPF $90 \%$ uncertainty bounds:

- Reliability: the number or percentage of daily observed streamflows within the HSPF 90\% uncertainty bounds;

- Sharpness: the width of the HSPF $90 \%$ uncertainty bounds (minimum, median, and maximum values).

The HSPF $90 \%$ confidence intervals were evaluated using daily observed flow data from $01 / 01 / 2003$ to $12 / 31 / 2005$ at the watershed outlet (USGS station 02443500). Three percentile classes of observed flows developed by the USGS (http://water.usgs.gov/waterwatch/) were calculated to find the effect of model Reliability to above normal ( $>75$ th percentile), normal (between 25 th and 75 th percentiles), and below normal flows ( $<25$ th percentile).

In addition to the Reliability and Sharpness criteria, continuous hydrographs of $90 \%$ uncertainty bounds and observed data were plotted. Scatterplots were drawn of 5th and 95th flow percentiles using the Monte Carlo and Harr scenarios. In this study, Monte Carlo simulations MCS were defined as benchmark. This method is widely considered as effective strategy for the evaluation of parametric uncertainty because the propagation of the parameter variability is performed using thousands of random numbers from the parameter distribution. On the other hand, the Harr method uses only 2 by the number of parameters to generate the uncertainty bounds. In Monte Carlo simulations, the computation of the sample size is a key factor (Haan, 2002). In this study, Monte Carlo simulations were made with sample sizes up to 5.000 runs. Then, the Absolute Relative Errors ARE (3) of simulated daily flows and number of Monte Carlo simulations were evaluated to find where the errors converge to a particular value.

Evaluation of the Harr method was accomplished in the same form as the MCS scenario (calculating the 95th and 5th percentiles of model outputs, and Reliability and Sharpness criteria). To compare the Monte Carlo results versus the Harr results, the Relative Error of Reliability $R E_{\text {Reliability }}$ and Sharpness $R E_{\text {Sharpness }}$ criteria values were calculated as follows:

$$
\begin{aligned}
& \operatorname{RE}_{\text {Re liability }}(\%)=\frac{(\text { Re liability })_{\text {Harr }}-(\text { Re liability })_{\text {Monte Carlo }}}{(\text { Re liability })_{\text {Monte Carlo }}} 100 \\
& \operatorname{RE}_{\text {Sharpness }}(\%)=\frac{(\text { Sharpness })_{\text {Harr }}-(\text { Sharpness })_{\text {Monte Carlo }}}{(\text { Sharpness })_{\text {Monte Carlo }}} 100
\end{aligned}
$$

\section{Results and discussion}

\section{Stability results of the Monte Carlo method}

Figure 2 shows the relationship between ARE of simulated daily flows and number of MCS. Each line in Figure 2 represents one simulation day between $01 / 01 / 2003$ and $12 / 31 / 2005$. This figure reveals that the $A R E$ values were close between runs 2.000 and 5.000. In general, higher MCS yielded lower $A R E$ results. It can be seen that the slope of the ARE values is steep for MCS less than 1.000 runs and getting flat between 2.000 and 5.000 runs. The 
median $A R E$ values from 3.000 to $5.000 \mathrm{MCS}$ were close $\left(5 \cdot 10^{-5}\right.$ to $\left.7 \cdot 10^{-5}\right)$. It took approximately 12 hours of CPU time to produce 5.000 simulations of the Luxapallila watershed model (with 115 NEXRAD grid points, one hour time step, and time simulation between 01/01/2002 and 12/31/2005) using a desktop computer with a $3.06 \mathrm{GHz}$ Xeon(TM) CPU and 1.00 GB of RAM. Therefore, 5.000 MCS, a median ARE value of $7 \cdot 10^{-5}$, and 12 hours of CPU time were considered sufficient for the purpose of this research.

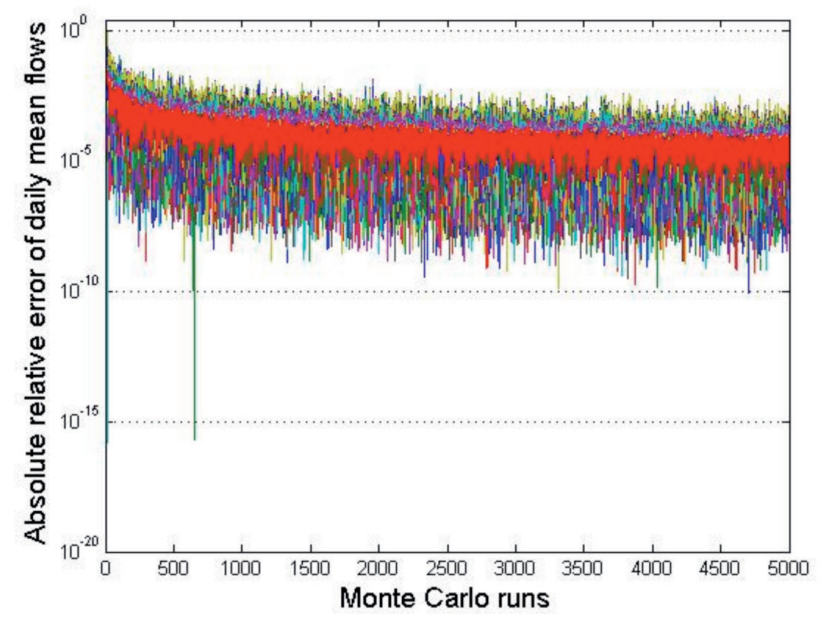

Figure 2: Stability of results using Monte Carlo method with Y-axis on a logarithmic scale

\section{Uncertainty estimates}

Results of parameter uncertainty propagation on HSPF model outputs using the Monte Carlo and Harr methods are shown in this section. Additionally, the Harr results were compared to the Monte Carlo results.

The Harr method used 24 simulations (two times the number of HSPF parameters) to solve the system, and normal distributions were used for the selected HSPF parameters. The baseline scenario using MCS used 5.000 interactions and triangular distributions to calculate the $90 \%$ uncertainty bounds.

Model Reliability results for the 2003-2005 period using the Monte Carlo and Harr methods were $65.4 \%$ and $72.7 \%$, respectively. This means that the Harr method uncertainty bounds included $11 \%$ more observed daily flows than the $90 \%$ uncertainty bounds yielded by the MCS. Relative errors of model Reliability results by observed flow percentiles from the Monte Carlo and Harr methods are shown in Table 5. The lowest and highest relative errors were $-0.8 \%$ and $18.6 \%$, respectively. Both uncertainty methods calculated close results for below normal flows. The Harr method consistently overestimated observed flows within the $90 \%$ uncertainty bounds for normal and above normal flows.

Table 5: Relative errors of model Reliability results by observed flow percentiles due to the Monte Carlo and Harr methods (2003-2005)

\begin{tabular}{cccc}
\hline Observed flow percentiles & $\begin{array}{c}\text { MCS } \\
\%\end{array}$ & $\begin{array}{c}\text { Harr } \\
\%\end{array}$ & $\begin{array}{c}\text { Relative error } \\
\%\end{array}$ \\
\hline$<25$ th & 92.3 & 91.6 & -0.8 \\
25th-75th & 60.2 & 71.4 & 18.6 \\
$>$ 75th & 48.2 & 55.8 & 15.9 \\
\hline
\end{tabular}

Figure 3 displays 5 th and 95th flow percentiles by the MCS and Harr methods. In general, high variability and a markedly high overestimation were shown by the Harr results.
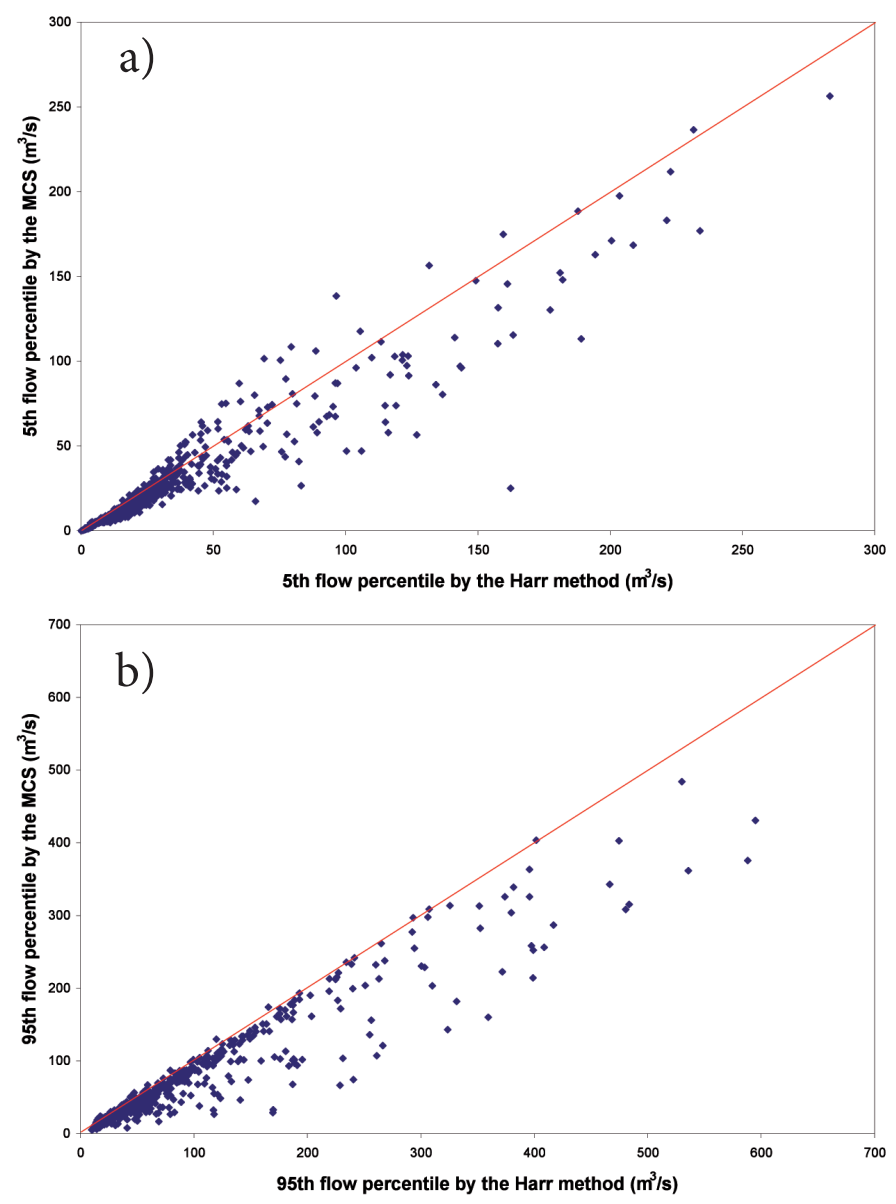

Figure 3: MCS and Harr methods (2003-2005), scatterplots of: a) 5 th flow percentile and b) 95 th flow percentile 
Figure 4 shows comparisons of uncertainty bounds generated by the MCS and Harr methods for selected storms. The uncertainty bounds calculated by both methods significantly differ from one another. All of the uncertainty bounds for peak flows by the Harr method were wider and higher than those yielded by the MCS. Table 6 shows selected percentiles of the model Sharpness calculated using the Monte Carlo and Harr methods. Clearly, the Harr method results overestimated the width of the $90 \%$ uncertainty bounds (model Sharpness) for each percentile.
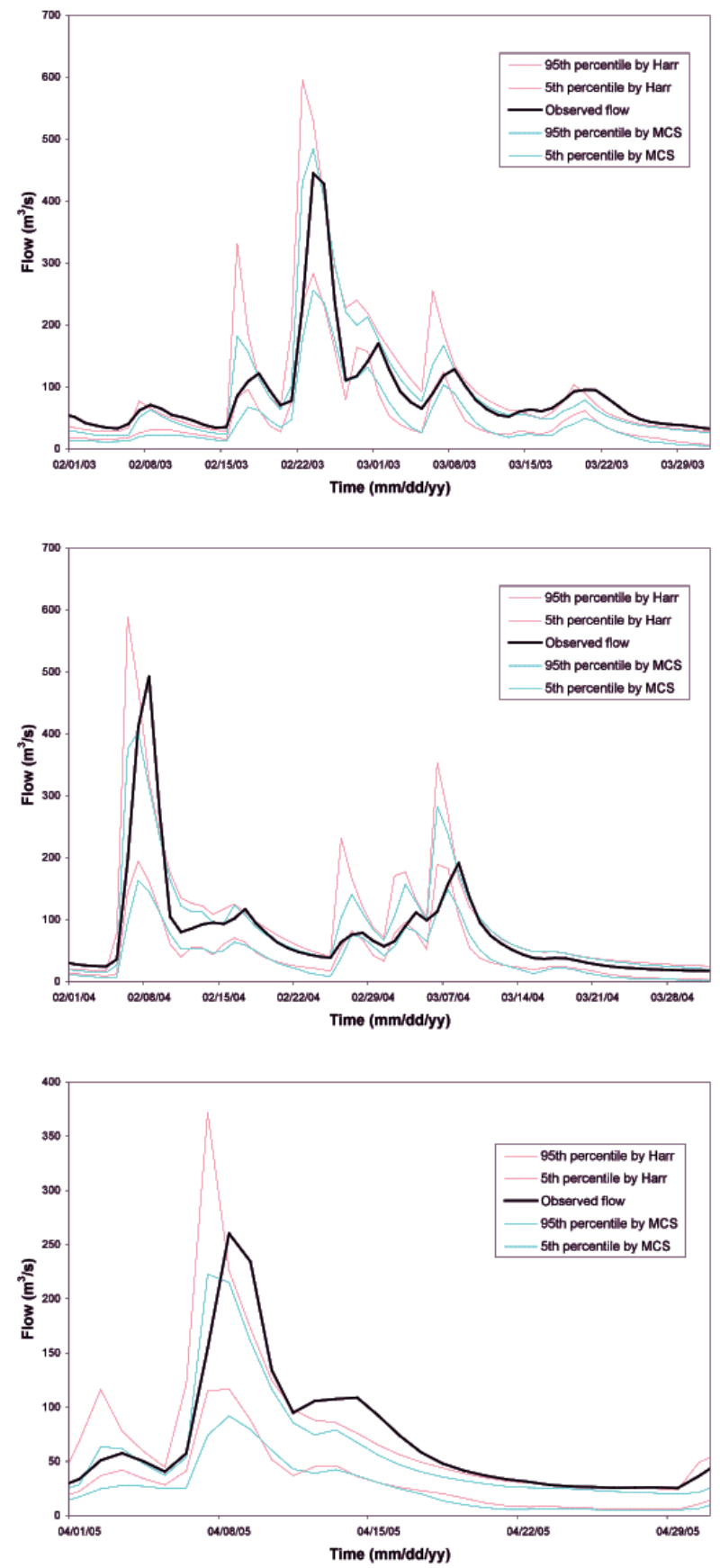

The minimum, median, and maximum relative errors of Sharpness criteria values between the MCS and Harr methods were $-43.6 \%, 20.7 \%$, and $563.6 \%$, respectively. The higher model Sharpness calculated using the Harr method may be explained by the use of just 24 Harr runs rather than the 5.000 Monte Carlo runs. Additionally, the Harr method selected the parameter values using normal distributions of the evaluated parameters rather than the triangular distributions used by the Monte Carlo method.
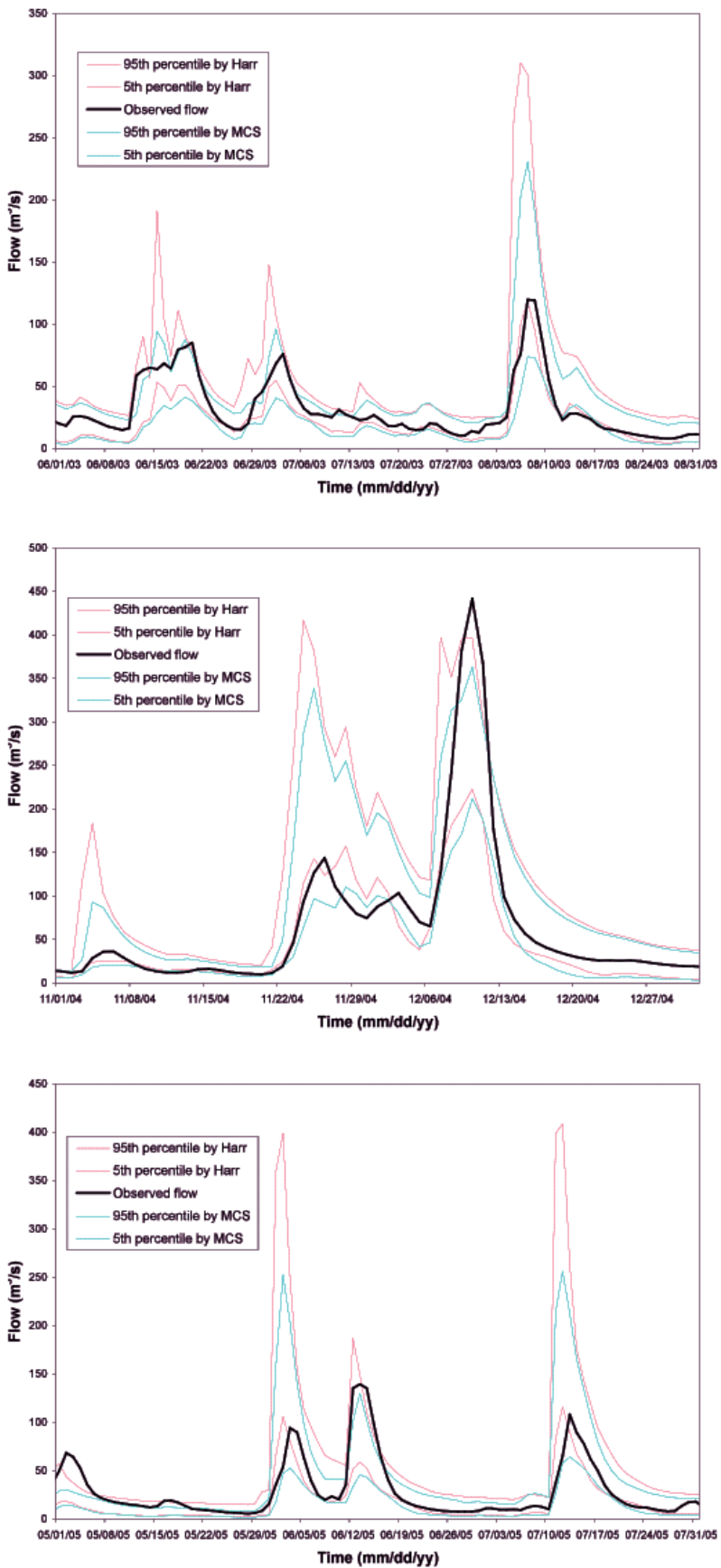

Figure 4: Daily observed hydrographs and uncertainty bounds estimated by the MCS and Harr methods 
Table 6: Selected percentiles of the range of the model Sharpness using the Monte Carlo and Harr methods

\begin{tabular}{ccc}
\hline Percentile & $\begin{array}{c}\text { MCS } \\
\mathrm{m}^{3} / \mathrm{s}\end{array}$ & $\begin{array}{c}\text { Harr } \\
\mathrm{m}^{3} / \mathrm{s}\end{array}$ \\
\hline Minimum & 4.8 & 7.1 \\
25th & 10.8 & 13.4 \\
50th & 16.2 & 19.1 \\
75th & 27.0 & 34.9 \\
Maximum & 336.7 & 444.4 \\
\hline
\end{tabular}

A drawback of the Harr method was the use of several parameter limit values rather than the calculated values to stay within the pre-established range of the HSPF parameters. Detailed definition of the 12 HSPF hydrology parameters used in this study is found in USEPA (2000). It was found that $16 \%$ of the coordinate values calculated by the Harr method were outside of the pre-defined parameter ranges (Table 7). Harr coordinate values out of range were changed by the closest limit value. HSPF parameters related to storm events, INTFW and IRC, yielded $20.8 \%$ and $16.7 \%$ respectively of values out of range. All of the new INTFW values were relocated to the lowest limit and, therefore, peak flows were expected to increase. IRC and AGWRC parameters control the rate at which interflow and groundwater, respectively, are discharged from storage. The KVARY parameter is used to describe non-linear groundwater recession rate. All of the new IRC and AGWRC values were changed to the highest limit and thus, slow flow rates in the recession limbs were estimated. The new KVARY values were relocated to the lowest limit and consequently, no seasonal variability of the groundwater flow was expected. DEEPFR, BASETP, AGWETP, CEPSC, and LZETP parameters control loss of water from the system and were replaced to the lowest value; therefore, streamflows were expected to increase.

In summary, the rearrangement of the Harr coordinates yielded more streamflow in the system and, therefore, the Harr model Sharpness was higher than the Monte Carlo results (baseline scenario). Christian and Baecher (2002) analyzed the problem of coordinate relocation in the Harr method for bounded parameters. They pointed out that "there seem to be no simple, elegant ways out of this dilemma." The use of other uncertainty methods (e.g. Rosenblueth's method or the Monte Carlo method) or reducing the number of random variables was recommended by the authors.
Table 7: Number of parameter values calculated out of range by the Harr method

\begin{tabular}{|c|c|c|c|c|}
\hline \multirow{2}{*}{ Parameter } & \multicolumn{2}{|c|}{$\begin{array}{c}\text { Relocation of the } \\
\text { coordinates }\end{array}$} & \multirow{2}{*}{$\begin{array}{l}\text { Number of } \\
\text { parameter } \\
\text { values out of } \\
\text { the range }\end{array}$} & \multirow{2}{*}{$\begin{array}{l}\text { Percentage } \\
\text { of total }\end{array}$} \\
\hline & Lowest & Highest & & \\
\hline LZSN & 1 & & 1 & 4.2 \\
\hline INFILT & & & 0 & 0.0 \\
\hline KVARY & 4 & & 4 & 16.7 \\
\hline AGWRC & & 2 & 2 & 8.3 \\
\hline DEEPFR & 9 & & 9 & 37.5 \\
\hline BASETP & 5 & & 5 & 20.8 \\
\hline AGWETP & 7 & & 7 & 29.2 \\
\hline CEPSC & 5 & & 5 & 20.8 \\
\hline UZSN & 1 & & 1 & 4.2 \\
\hline INTFW & 5 & & 5 & 20.8 \\
\hline IRC & & 4 & 4 & 16.7 \\
\hline LZETP & 3 & 1 & 4 & 16.7 \\
\hline
\end{tabular}

\section{Summary and conclusions}

Variability of 12 HSPF hydrology parameters was propagated through the USEPA HSPF model to compute the $90 \%$ uncertainty bounds of daily streamflow simulations using the Harr probabilistic point estimate method and Monte Carlo simulation. This study used physical data from a watershed in Alabama and Mississippi, USA. The Harr method is a very simple uncertainty method that only uses the mean, standard deviation, and correlation of symmetric variables. The Monte Carlo method is a straightforward uncertainty method that is computationally inefficient for large sets of data and requires the description of the probability distribution function.

In this study, reasonable estimates of mean and standard deviation of 12 HSPF parameters were obtained from a database with 27 model applications in USA. It was shown that the variables were roughly symmetric meaning that a probabilistic definition can be achieved using the Harr method. The Harr method calculated 11\% more observed flows within the $90 \%$ uncertainty bounds than the Monte Carlo results. Computational efficiency was improved, using 24 runs (two minutes) with Harr's method to estimate the HSPF 90\% uncertainty bounds versus 5.000 runs (12 hours) using the Monte Carlo method. A drawback of the Harr method was the use of several parameter limit values instead of the calculated value to keep the pre-established range of the HSPF parameters. In some parameters around $30 \%$ of values were changed and the rearrangement of the 
Harr coordinates yielded more streamflow in the system. Therefore, the model Sharpness was wider with the Harr results than with the Monte Carlo results (median relative errors around 20\% were calculated for model Sharpness). Model Sharpness was wider with the Harr method because it forced extreme values of each parameter to be sampled with the same or higher frequency as the central values, thus exploring a broader range of HSPF outputs than those generated using the Monte Carlo method (triangular distributions). The Harr method Sharpness bias was fairly constant throughout the different flows (below normal, normal, and above normal); however, the model Reliability results were variable throughout the different flows, with relative errors of $-0.8 \%, 18.6 \%$, and $15.9 \%$ for below normal, normal, and above normal flows, respectively.

The comparison showed that Harr's method could be an appropriate initial indicator of parameter uncertainty propagation on streamflow simulations, in particular on hydrology models with several parameters and high spatial discretization (multidimensional grid models). The Monte Carlo simulations are recommended when knowledge (probability distribution functions of variables) and computational resources (in terms of computational power to solve large sets of data) are feasible. More research is needed to find appropriate estimates of statistical moments (Harr method) and probability distribution function (Monte Carlo method) of model parameters that could improve uncertainty method results.

\section{Acknowledgements}

This work was performed with funding from the Northern Gulf Institute project 07-MSU-03 Watershed Modeling Improvements to Enhance Coastal Ecosystems. The writers appreciate the contribution of Cecilia Hernandez, a City University of New York PhD student who developed the MATLAB codes to uncompress and generate time series of NEXRAD rainfall data. The Kelly Gene Cook foundation supported Camacho's graduate assistantship.

\section{References}

Al-Abed, N.A. and Whiteley, H.R. (2002). Calibration of the hydrological simulation program fortran (HSPF) model using automatic calibration and geographical information systems. Hydrological Process 16, 3169-3188

Alarcon V.J., McAnally, W., Diaz-Ramirez, J., Martin, J. and
Cartwright, J. (2009). A hydrological model of the Mobile River watershed, Southeastern USA. AIP Conference Proceedings 1148, 641-645

Albek, M., Öğütveren, Ü.B. and Albek, E. (2004). Hydrological modeling of Seydi Suyu watershed (Turkey) with HSPF. Journal of Hydrology 285, 260-271

Beven, K. and Binley, A. (1992). The future of distributed models: model calibration and uncertainty prediction. Hydrological Processes 6(3), 279-298

Bicknell, B.R., Imnoff, J.C., Jobes, T.H. and Donigian, A.S. (2001). Hydrological Simulation Program - Fortran HSPF version 12, User's Manual. Prepared for AQUA TERRA Consultants Mountain View, California, in co-operation with Water Resources Discipline U.S. Geological Survey Reston, Virginia, and U.S. Environmental Protection Agency Athens, Georgia

Binley, A.M., Beven, K.J., Calver, A. and Watts, L.G. (1991). Changing responses in hydrology: assessing the uncertainty in physically based model predictions. Water Resources Research 27(6), 1253-1261

Butts, M.B., Payne, J.T., Kristensen, M. and Madsen, H. (2004). An evaluation of the impact of model structure on hydrological modelling uncertainty for streamflow simulation. Journal of Hydrology 298, 242-266

Carpenter, T.M. and Georgakakos, K.P. (2004). Impacts of parametric and radar rainfall uncertainty on the ensemble streamflow simulations of a distributed hydrologic model. Journal of Hydrology 298, 202-221

Christian, J.T. and Baecher, G.B. (2002). The point-estimate method with large numbers of variables. International Journal for Numerical and Analytical Methods in Geomechanics 26, $1515-1529$

Diaz-Ramirez, J.N., Perez-Alegria, L.R. and McAnally. W.H. (2008). Hydrology and sediment modeling using HSPF/BASINS in a tropical island watershed. Transactions of the ASABE 51(5), $1555-1565$

Diaz-Ramirez, J.N., McAnally, W.H. and Martin, J.L. (2011). Analysis of hydrological processes applying the HSPF model in selected watersheds in Alabama, Mississippi, and Puerto Rico. Applied Engineering in Agriculture 27(6), 937-954

Dilks, D.W., Canale, R.P. and Meier, P.G. (1992). Development of Bayesian Monte Carlo techniques for water quality model uncertainty. Ecological Modelling 62, 149-162

Donigian, A.S., Bicknell, B.R. and Imhoff, J.C. (1995). Hydrological Simulation Program - FORTRAN (HSPF). In: 
Computer Models of Watershed Hydrology. Chapter 12, V.P. Sigh (editor). Water Resources Publications

Duan, Z., Diaz, J.N., Martin, J.L. and McAnally, W.H. (2008). Effects of land-use changes on Saint Louis Bay watershed modeling. Journal of Coastal Research, Special Issue 52, 117-124

Georgakakos, K.P., Seo, D.J. Gupta, H., Schaake, J. and Butts, M.B. (2004). Towards the characterization of streamflow simulation uncertainty through multimodel ensembles. Journal of Hydrology 298, 222-241

Gupta, H.V., Beven, K.J. and Wagener, T. (2005). Model Calibration and Uncertainty Estimation. In: Encyclopedia of Hydrological Sciences, M. Anderson (editor). John Wiley \& Sons Ltd.

Haan, C.T. (2002). Statistical Methods in Hydrology. 2nd ed., Iowa State Press

Harr, M.E. (1989). Probabilistic estimates for multivariate analysis. Applied Mathematical Modelling 13, 313-318

Hayashi, S., Murakami, S., Watanabe, M. and Bao-Hua, X. (2004). HSPF simulation of runoff and sediment loads in the upper Changjiang River Basin, China. Journal of Environmental Engineering 130(7), 801-815

Hummel, P., Kittle, J. and Gray, M. (2001). WDMUtil User's Manual Version 2.0: A Tool for Managing Watershed Modeling Time-Series Data. U.S. Environmental Protection Agency, Office of Science and Technology and Office of Water, Washington, D.C.

Jia, Y. ( 2004). Robust Optimization for Total Maximum Daily Load Allocations. Ph.D. dissertation, University of Virginia

Laroche, A., Gallichand, J., Lagacé, R. and Pesant, A. (1996). Simulating atrazine transport with HSPF in an agricultural watershed. Journal of Environmental Engineering 122(7), 622-630

McAnally, W.H., Martin, J.L., Diaz, J.N., Duan, Z., Mancilla, C.A., Tagert, M.L., O’Hara, C. and Ballweber, J.A. (2006). Assimilating Remotely Sensed Data into Hydrologic Decision Support Systems: BASINS Evaluation. Department of Civil Engineering and GeoResources Institute, Mississippi State University, MS

McIntyre, N., Wheater, H. and Lees, M. (2002). Estimation and propagation of parametric uncertainty in environmental models. Journal of Hydroinformatics 4(3), 177-197

Melching, C.S. (1992). An improved first-order reliability approach for assessing uncertainties in hydrologic modeling. Journal of Hydrology 132, 157-177

Melching, C.S. (1995). Reliability Estimation. In: Computer Models of Watershed Hydrology, Chapter 3, V.P. Sigh (editor).
Water Resources Publications, Littleton, CO

Metropolis, N. and Ulam, S. (1949). The Monte Carlo method. Journal of the American Statistical Association 44(247), 335-341

Moore, L.W., Matheny, H., Tyree, T., Sabatini, D. and Klaine, S.J. (1988). Agricultural runoff modeling in a small west Tennessee watershed. Research Journal of the Water Pollution Control Federation 60(2), 242-249

Morgan, M.G. and Henrion, M. (1990). Uncertainty: A Guide to Dealing with Uncertainty in Quantitative Risk and Policy Analysis. Cambridge University Press

Nasr, A., Bruen, M., Jordan, P., Moles, R., Kiely, G. and Byrne. P. (2007). A comparison of SWAT, HSPF and SHETRAN/GOPC for modelling phosphorus export from three catchments in Ireland. Water Research 41(5), 1065-1073

Paul, S. (2003). Bacterial Total Maximum Daily Load (TMDL): Development and Evaluation of a New Classification Scheme for Impaired Waterbodies of Texas. Ph.D. dissertation, Texas A\&M University

Refsgaard, J.C., and Henriksen, H.J. (2004). Modelling guidelines-terminology and guiding principles. Advances in Water Resources 27(1):71-82

Refsgaard, J.C., van der Sluijs, J.P., Hojberg, A.L. and Vanrolleghem, P.A. (2007). Uncertainty in the environmental modelling process - a framework and guidance. Environmental Modelling and Software 22, 1543-1556

Rogers, C.C.M., Beven, K.J., Morris, E.M. and Anderson, M.G. (1985). Sensitivity analysis, calibration and predictive uncertainty of the institute of hydrology distributed model. Journal of Hydrology 81, 179-191

Ronen, Y. (1988). The Role of Uncertainties. In: Uncertainty Analysis, Y. Ronen (ed.). CRC Press

Rosenblueth, E. (1975). Point estimates for probability moments. Proceedings of the National Academy of Sciences of the United States of America 72(10), 3812-3814

Singh, V.P. (1995). Computer Models of Watershed Hydrology. Water Resources Publications

Singh, V.P. and Woolhiser, D.A. (2002). Mathematical modeling of watershed hydrology. Journal of Hydrology Engineering 7(4), 270-292

Singh, V.P. and Frevert, D.K. (2002). Mathematical Models of Large Watershed Hydrology. Water Resources Publications

Sobol', I.M. (1994). A Primer for the Monte Carlo Method. CRC Press 
Souid, M.A. (1999). Reliability of Rainfall-Runoff Models. Ph.D. dissertation, State University of New York

Thiemann, M., Trosset, M., Gupta, H. and Sorooshian, S. (2001). Bayesian recursive parameter estimation for hydrologic models. Water Resources Research 37(10), 2521-2535

Tung, Y.K. (1996). Uncertainty and Reliability Analysis. In: Water Resources Handbook, L.W. Mays (editor). McGraw-Hill

Tung, Y.K. and Yen, B.C. (2005). Hydrosystems Engineering Uncertainty Analysis. McGraw-Hill

USEPA US Environmental Protection Agency (2000). BASINS technical note 6: estimating hydrology and hydraulic parameters for HSPF. Available at: http://www.epa.gov/waterscience/ basins/docs/tecnote6.pdf. Accessed in November 2008

USEPA US Environmental Protection Agency (2006). HSPFParm version 1.3 beta July 2002. Available at: http://www. epa.gov/OST/ftp/basins/HSPFParm/. Accessed in April 7, 2006

USEPA US Environmental Protection Agency (2011). Better assessment science integrating point and nonpoint sources (BASINS) web site. Available at http://water.epa.gov/scitech/ datait/models/basins/index.cfm. Accessed in November 2011
USEPA US Environmental Protection Agency (2012). Flow calibration tutorial: calibration scenarios. Available at: http:// water.epa.gov/scitech/datait/models/basins/scenario.cfm. Accessed in June 13, 2012

Wagener, T., McIntyre, N., Lees, M.J., Wheater, H.S. and Gupta, H.V. (2003). Towards reduced uncertainty in conceptual rainfallrunoff modelling: dynamic identifiability analysis. Hydrological Processes 17(2), 455-476

Wu, J. (2004). Water-Quality-Based BMP Design Approach and Uncertainty Analysis for Integrated Watershed Management. Ph.D. dissertation, University of Virginia

Zhang, H.X. and Yu, S.L. (2004). Applying the first-order error analysis in determining the margin of safety for total maximum daily load computations. Journal of Environmental Engineering 130(6), 664-673

Yu, P.S., Yang, T.C. and Chen, S.J. (2001). Comparison of uncertainty analysis methods for a distributed rainfall-runoff model. Journal of Hydrology 244, 43-59 